Universidade de São Paulo

Instituto de Astronomia, Geofísica e Ciências Atmosféricas

Departamento de Astronomia

\author{
Aiara Lobo Gomes
}

\title{
A Estrutura do Campo Magnético na Pequena Nuvem de Magalhães
}

São Paulo 



\author{
Aiara Lobo Gomes
}

\title{
A Estrutura do Campo Magnético na Pequena Nuvem de Magalhães
}

Dissertação apresentada ao Departamento de Astronomia do Instituto de Astronomia, Geofísica e Ciências Atmosféricas da Universidade de São Paulo como requisito parcial para a obtenção do título de Mestre em Ciências.

Área de Concentração: Astronomia

Orientador: Prof. Dr. Antônio Mário Magalhães

Versão corrigida

São Paulo 

Nota: A versão original desta dissertação encontra-se disponível no Serviço de Biblioteca e Documentação do Instituto de Astronomia, Geofísica e Ciências Atmosféricas IAG/USP. 

Aos meus pais: Antonio e Maria;

Às minhas irmãs: Leo, Li e Mile; Aos meus sobrinhos: Nhão e Toninha; Aos litros de café... 



\section{Agradecimentos}

Aos meus pais, minhas irmãs, meus pitchuquinhos (sobrinhos), meus cunhados e toda minha família, pelo amor, compreensão, incentivo e ajuda durante toda a minha vida;

A mi niño (Juanito), pelo companheirismo, carinho, interesse, apoio e paciência;

A todos os amigos e colegas, por toda ajuda ou simplesmente por estarem sempre por perto. Saibam que os jogos de RPG e parqués foram essenciais nessa fase fim de tese. Em especial ao Caio, Cói, Daizinha, Dani, Fred, Greg, Jose, Kazuo, Má, Marcelitos, Ná, Thaíse, Reinaldo e Xavier;

Ao meu orientador Antônio Mário, pela confiança, paciência e por tudo que me ensinou;

A todos os professores que participaram da minha formação, especialmente à Bel (IME) e à Bete (IAG), por serem professoras brilhantes e particularmente gentis;

À Dai, Dani, Juan, Lizinha (em especial!) e Thaíse, pela ajuda com as correções de português e/ou leitura crítica dessa dissertação;

Ao Antonio Pereyra, por sempre responder com paciência às dúvidas sobre o IRAF;

À Snezana Stanimirović, por ceder gentilmente as imagens de HI e sempre responder com prontidão às nossas questões;

Aos funcionários do IAG, por sempre estarem dispostos a ajudar no que fosse necessário;

Aos funcionários do OPD e CTIO, por toda a atenção durante as missões observacionais;

À FAPESP, pelo apoio financeiro sob o projeto $n^{o}$ : 2010/03802-2 (08/10 até 02/12), e ao $\mathrm{CNPq}$, pelo apoio financeiro sob o projeto $\mathrm{n}^{o}$ 132834/2010-3 (03/10 até 07/10);

À FAPESP, CNPq, PROEX, CPq (IAG) e Jagiellonian University, pelo apoio financeiro para ida a congressos, escolas e missões observacionais;

Por último, e não menos importante, "vaaaaiiii Cooorinnnnthiannnsss!!!".

* Nota: Esta dissertação foi escrita em $\mathrm{H}_{\mathrm{T}} \mathrm{E}$ X com a classe IAGTESE, para teses e dissertações do IAG. 

"Há sem dúvida quem ame o infinito, Há sem dúvida quem deseje o impossível, Há sem dúvida quem não queira nadaTrês tipos de idealistas, e eu nenhum deles:

Porque eu amo infinitamente o finito, Porque eu desejo impossivelmente o possivel, Porque eu quero tudo, ou um pouco mais, se puder ser, Ou até se não puder ser..."

Álvaro de Campos (heterônimo de Fernando Pessoa) 



\section{Resumo}

A Pequena Nuvem de Magalhães (PNM) é uma galáxia irregular e rica em gás, que juntamente com a Grande Nuvem de Magalhães (GNM) orbita a Via Láctea (VL). Elas formam um sistema triplo em constante interação. A PNM possui metalicidade baixa, e consequentemente seu meio interestelar (MI) apresenta propriedades particularmente diferentes das observadas para o MI da Galáxia. Mais do que isso, a importância do campo magnético em escalas galácticas vem sendo evidenciada cada vez mais. Então, o objetivo desta dissertação foi estudar a estrutura do campo magnético na PNM, e sua relação com componentes do MI desta galáxia.

Para este fim, utilizamos dados de polarimetria no óptico, obtidos no Cerro Tololo Inter-American Observatory. Construímos um catálogo polarimétrico que contém 7.207 estrelas em 28 campos distribuídos nas secções Nordeste e da Asa da PNM. Os mapas de polarização traçam o campo magnético no plano do céu diretamente, e pode-se obter sua intensidade utilizando o método de Chandrasekhar \& Fermi. A partir do catálogo polarimétrico gerado neste trabalho, conseguimos observar que o campo magnético na PNM possui direção bastante irregular, porém é provável a existência de dois padrões em larga escala - o primeiro alinhado com a Ponte pan-Magelânica e o segundo alinhado com a Barra da PNM. Obtivemos para o campo magnético regular $B_{\text {céu }}=(1,84 \pm 0,11) \mu \mathrm{G}$ e para o campo turbulento $\delta B=(2,920 \pm 0,098) \mu \mathrm{G}$. Esse resultado evidencia que na PNM o campo aleatório domina com relação ao de larga escala, justificando a observação de uma configuração tão irregular para os vetores de polarização.

Correlacionando os mapas de polarização com estruturas presentes no MI da PNM, pudemos verificar a presença de diversos shells que podem possuir campos magnéticos da ordem de algumas dezenas de $\mu \mathrm{G}$. Também foi possível observar ambientes onde o campo 
regular parece ter sido destruído pela turbulência e outros onde ele pode ainda não ter tido tempo de se formar.

Derivamos a relação entre polarização e avermelhamento, e obtivemos como resultado que ela é da ordem de $P / A_{V} \simeq 2$, o que indica que na PNM a eficiência para polarização pode ser menor do que na Galáxia, talvez devido a alta turbulência e/ou ao fato de que nela o campo regular é muito baixo. Por fim, a partir da estimativa para as densidades de energia do campo magnético e para o movimento de rotação e de turbulência do gás, pudemos mostrar que o campo magnético possui importância dinâmica para PNM, sendo a componente turbulenta a maior responsável pela pressão magnética. 


\section{Abstract}

The Small Magellanic Cloud (SMC) is a gas rich irregular galaxy which, together with the Large Magellanic Cloud (LMC), orbit the Milky Way (MW). They form a triple system in constant interaction. The SMC is a metal poor galaxy and, due to this, its interstellar medium (ISM) presents different properties from the Galaxy's ISM. In addition to that, the importance of magnetic fields on galactic scales is being recognized nowadays. Therefore, the aim of this project was to study the magnetic field structure of the SMC and its relationship with other components of SMC's ISM.

For this purpose we have used starlight optical polarimetric data, obtained at Cerro Tololo Inter-American Observatory. We have constructed a polarization catalog containing a total of 7,207 stars in 28 fields in the Northeast/Wing sections of the SMC. The polarimetric vector maps trace the ISM magnetic field component in the plane of the sky and one can estimate its intensity towards a given region using the Chandrasekhar \& Fermi method. Making use of the polarimetric catalog from this work, we have found that the magnetic field in the SMC, although varying from region to region, nevertheless shows two large scale patterns - the first one aligned with the Magellanic Bridge and a second one aligned with the SMC's Bar. We derived for the regular sky-projected magnetic field a value of $B_{\text {sky }}=(1.84 \pm 0.11) \mu \mathrm{G}$, and for the turbulent magnetic field $\delta B=(2.920 \pm 0.098) \mu \mathrm{G}$. These results evidence that in the SMC the random field prevails over the large scale field, which explains the irregular configuration of the polarization vectors often seen.

Correlating the polarization maps with structures present on the SMC's ISM, we could identify the presence of several shells which may have magnetic fields up to a few tens $\mu \mathrm{G}$. It was also possible to observe environments where the regular field seems to have been destroyed due to turbulence, and others where it seems that the large scale magnetic field 
has not enough time to be formed.

Studying the relationship with polarization and reddening, we have obtained a value for $P / A_{V} \simeq 2$, which may indicate that the polarization efficiency in the SMC is smaller than in the Galaxy, perhaps due to a higher turbulence and/or because of a smaller regular magnetic field. Lastly, we have estimated the energy density for the magnetic field and for the rotation and turbulent gas motions. We showed that the magnetic field is dynamically important in the SMC's ISM, and that the turbulent component is the largest contributor to the magnetic pressure. 


\section{Lista de Figuras}

1.1 Representação dos estados de polarização. . . . . . . . . . . . . . . . . . . 28

1.2 Mecanismos físicos que causam polarização. . . . . . . . . . . . . . . . . . 29

1.3 Desenho esquemático de filtros que determinam os parâmetros de Stokes. . 30

1.4 Ilustração de como a polarização interestelar é causada. . . . . . . . . . . . . 32

1.5 Encurvamento das linhas de campo. . . . . . . . . . . . . . . . 36

1.6 Imagem composta da PNM. . . . . . . . . . . . . . . . . . . . 38

1.7 Curvas de extinção para a PNM. . . . . . . . . . . . . . . . . . . 43

2.1 Lâmina retardadora de meia-onda. . . . . . . . . . . . . . . . . . . . . 46

2.2 Configuração instrumental que pode ser utilizada para medir o grau de polarização linear de um feixe de luz. . . . . . . . . . . . . . . . . . 47

2.3 Campos observados na PNM. . . . . . . . . . . . . . . . . . . . . 48

2.4 Correções $\Delta m_{V}$ para magnitude para cada um dos campos. . . . . . . . . . 61

2.5 Erros nas coordenadas para cada campo. . . . . . . . . . . . . . 62

3.1 Divisão da PNM feita por Schmidt (1976). . . . . . . . . . . . . . . . . . . 64

3.2 Diagrama HR retirado de Karttunen et al. (2007) . . . . . . . . . . . . . . . 66

3.3 Gráficos por região dos parâmetros de Stokes Q e U das estrelas selecionadas como candidatas a serem de foreground. . . . . . . . . . . . . . . . 68

3.4 Gráficos por região dos parâmetros de Stokes Q e U das estrelas selecionadas como candidatas a serem de foreground (continuação). . . . . . . . . . . . 69

3.5 Histogramas para $P_{\text {cor }} / P_{\text {obs }}$ e $\theta_{\text {cor }}-\theta_{\text {obs }} \ldots \ldots \ldots$. . . . . . . . . 71

3.6 Histogramas para as magnitudes na banda V. . . . . . . . . . . . . . . 73

3.7 Gráficos da polarização versus magnitude. . . . . . . . . . . . . . . . 75 
3.8 Exemplos de campos com apenas um padrão para $\theta$. Histogramas para o ângulo de polarização e a sua intensidade. . . . . . . . . . . . . . . . . 77

3.9 Exemplo de campo com dois padrões para $\theta$. Histogramas para o ângulo de polarização e a sua intensidade. . . . . . . . . . . . . . . . . 78

3.10 Exemplos de campos com nenhum padrão para $\theta$. Histogramas para o ângulo de polarização e a sua intensidade. . . . . . . . . . . . . . . . . . . 79

3.11 Exemplo de campo filtrado por $m_{V}$. Histogramas para o ângulo de polarização e a sua intensidade, e gráfico de $\theta$ vs $m_{V}$. . . . . . . . . . . . 80

3.12 Exemplos de campos com estrelas excluídas para ajuste do padrão de $\theta$. Histogramas para o ângulo de polarização e a sua intensidade. . . . . . . . 81

3.13 Mapa dos padrões de polarização. . . . . . . . . . . . . . . . . 82

3.14 Mapa dos padrões que possuem menor intensidade de polarização. . . . . . 82

3.15 Histogramas para $\theta_{M} \ldots \ldots \ldots \ldots \ldots$. . . . . . . . . . . . . 84

3.16 Mapa de dispersão de velocidade do HI na linha de visada. . . . . . . . . . 86

3.17 Mapa de densidade de coluna de HI. . . . . . . . . . . . . . . . . . . . 87

4.1 Mapas de polarização para os campos PNM 09 e 11. . . . . . . . . . . . 92

4.2 Mapa de polarização para o campo PNM06. . . . . . . . . . . . . . . . . . 93

4.3 Avermelhamento em função da mediana da polarização de cada padrão. . . 95

A.1 Campos com apenas um padrão para $\theta$. Histogramas para o ângulo de polarização e a sua intensidade.

A.2 Campos com apenas um padrão para $\theta$. Histogramas para o ângulo de polarização e a sua intensidade (continuação). . . . . . . . . . . . . . . . . 116

A.3 Campos com dois padrões para $\theta$. Histogramas para o ângulo de polarização e a sua intensidade.

A.4 Campos com dois padrões para $\theta$. Histogramas para o ângulo de polarização e a sua intensidade (continuação).

A.5 Campos com nenhum padrão para $\theta$. Histogramas para o ângulo de polarização e a sua intensidade.

A.6 Campos filtrados por $m_{V}$. Histogramas para o ângulo de polarização e a sua intensidade, e gráfico de $\theta$ vs $m_{V} \ldots \ldots \ldots \ldots$. . . . . . . . . 
A.7 Campos com estrelas excluídas para o ajuste do padrão de $\theta$. Histogramas para o ângulo de polarização e a sua intensidade. . . . . . . . . . . . . . . 121

B.1 Mapa de polarização. . . . . . . . . . . . . . . . . . . . . . . . . . . 123

B.2 Mapas de polarização (continuação). . . . . . . . . . . . . . . . . . 124

B.3 Mapas de polarização (continuação). . . . . . . . . . . . . . . . 125

B.4 Mapas de polarização (continuação). . . . . . . . . . . . . . . . . 126

B.5 Mapas de polarização (continuação). . . . . . . . . . . . . . . . . . . 127

B.6 Mapas de polarização (continuação). . . . . . . . . . . . . . . . . . . 128

B.7 Mapas de polarização (continuação). . . . . . . . . . . . . . . . . . . . 129

B.8 Mapas de polarização (continuação). . . . . . . . . . . . . . . . . . . . . 130

B.9 Mapas de polarização (continuação). . . . . . . . . . . . . . . . . . . 131 



\section{Lista de Tabelas}

1.1 Exemplos astrofísicos para diversos mecanismos de polarização da luz. . . . 29

2.1 Parâmetros das imagens de bias e flat-field. . . . . . . . . . . . . . . . . . 47

2.2 Informações referente as estrelas padrões. . . . . . . . . . . . . . . . . . 48

2.3 Coordenadas dos campos e noite em que foram observados. . . . . . . . . . 49

2.4 Trimsec e biassec para cada seção do CCD utilizada. . . . . . . . . . . . . 50

2.5 Lista de parâmetros de entrada para a tarefa quickpol. . . . . . . . . . . . . 52

2.6 Parâmetros polarimétricos das estrelas padrões. . . . . . . . . . . . . . . . 54

2.7 Lista dos parâmetros fixos dados como entrada para a tarefa refer. . . . . . 58

2.8 Informações referente a calibração de magnitudes. . . . . . . . . . . . . . 60

3.1 Regiões de Schmidt (1976) às quais cada campo pertence. . . . . . . . . . . . 64

3.2 Estimativas da polarização de foreground para cada região da PNM. . . . . 69

3.3 Parâmetros locais do MI dos nossos campos na PNM. . . . . . . . . . . . 88

4.1 Origem dos padrões de polarização/campo magnético de cada campo. . . . 94

4.2 Parâmetros médios para o gás e o campo magnético na PNM. . . . . . . . 98

4.3 Densidades de energia médias na PNM. . . . . . . . . . . . . . . . . . . . . 98

A.1 Parâmetros dos padrões de polarização. . . . . . . . . . . . . . . . . . . 122

B.1 Lista de objetos relacionados ao MI, presentes em cada campo CCD. . . . 132 



\section{Sumário}

1. Introdução . . . . . . . . . . . . . . . . . . . . . . . . . 25

1.1 O Meio Interestelar . . . . . . . . . . . . . . . . . . . . 26

1.2 Polarização da Luz . . . . . . . . . . . . . . . . . . . . . . . . . . . . 27

1.2.1 Os Parâmetros de Stokes . . . . . . . . . . . . . . . . . . 29

1.2.2 Polarização pelo Meio Interestelar . . . . . . . . . . . . . . . . . . . 31

1.3 O Método de Chandrasekhar \& Fermi . . . . . . . . . . . . . . . . . . . . 33

1.3.1 Método CF Modificado . . . . . . . . . . . . . . . . . . . 35

1.4 Nuvens de Magalhães . . . . . . . . . . . . . . . . . . . . . . . . . . . . . . 37

1.4.1 A Pequena Nuvem de Magalhães . . . . . . . . . . . . . . . . . . . 37

1.4.1.1 Radiação Ultravioleta . . . . . . . . . . . . . . . . 38

1.4.1.2 Campo Magnético . . . . . . . . . . . . . . . . 38

1.4.1.3 Hidrogênio Ionizado . . . . . . . . . . . . . . . . 39

1.4.1.4 Hidrogênio Neutro . . . . . . . . . . . . . . . . . . . . 39

1.4.1.5 Gás Molecular . . . . . . . . . . . . . 40

1.4.1.6 Poeira ..................... . . . 41

1.4.2 A Ponte pan-Magelânica . . . . . . . . . . . . . . . . . . . 44

2. Dados Observacionais . . . . . . . . . . . . . . . . . . . 45

2.1 Polarimetria de Imagem Óptica . . . . . . . . . . . . . . . . . . 45

2.2 Observações . . . . . . . . . . . . . . . . . . . . . . . . 47

2.3 Redução dos Dados . . . . . . . . . . . . . . . . . . . . . . . . 49

2.3 .1 Tratamento das Imagens . . . . . . . . . . . . . . . . . . . . . 49 
2.3.2 Parâmetros Polarimétricos . . . . . . . . . . . . . . . 51

2.3.2.1 Estrelas Padrões . . . . . . . . . . . . . . . 54

2.3.2.2 Campos na PNM . . . . . . . . . . . . 55

2.3.3 Catálogos Polarimétricos . . . . . . . . . . . . 57

2.3.3.1 Calibração das Posições . . . . . . . . . . . . . . . 57

2.3.3.2 Obtenção das Magnitudes e Catálogos . . . . . . . . . . 59

2.3.3.3 Estimativa dos Erros para Ascensão Reta e Declinação . . 61

3. Estrutura do Campo Magnético na PNM . . . . . . . . . . . . . . . 63

3.1 Polarização de Foreground . . . . . . . . . . . . . . . . . . . . . 63

3.1.1 Determinação da Polarização de Foreground . . . . . . . . . . . . . 65

3.1.2 Subtração da Polarização de Foreground . . . . . . . . . . . . . . . 70

3.2 Análise do Catálogo Polarimétrico . . . . . . . . . . . . . . . . 72

3.2.1 Magnitudes na Banda V . . . . . . . . . . . . . . . . . . 72

3.2 .2 Polarização versus Magnitude . . . . . . . . . . . . . . . . . . . . 74

3.3 Mapa de Polarização . . . . . . . . . . . . . . . . . . . . 76

3.3.1 Campos com Um Padrão . . . . . . . . . . . . . . . . . . . 76

3.3.2 Campos com Dois Padrões . . . . . . . . . . . . . . . . . . . 78

3.3.3 Campos com Nenhum Padrão . . . . . . . . . . . . . . . . . 78

3.3.4 Campos Filtrados por $m_{V} \ldots \ldots \ldots \ldots$. . . . . . . . 79

3.3.5 Campos com Estrelas Excluídas . . . . . . . . . . . . . . . . 80

3.3.6 Mapa de Polarização . . . . . . . . . . . . . . . . . . . . . 81

3.4 Determinação da Intensidade do Campo Magnético . . . . . . . . . . . . . 85

3.4.1 Dispersão de Velocidade do HI . . . . . . . . . . . . . . . . . . 85

3.4 .2 Densidade de Massa do HI . . . . . . . . . . . . . . . . . . . . 86

3.4 .3 Aplicação do Método CF . . . . . . . . . . . . . . . . . 87

4. O Meio Interestelar da PNM . . . . . . . . . . . . . . . . . . . . . . . . . . . . . 91

4.1 Correlação entre os Padrões de Polarização e Estruturas do MI . . . . . . . 91

4.2 Relação entre Polarização e Avermelhamento . . . . . . . . . . . . . . . . . 94

4.3 Importância Dinâmica do Campo Magnético . . . . . . . . . . . . . . . . 97

5. Conclusões e Perspectivas . . . . . . . . . . . . . . . . . . . . . . . 101 
Apêndice

A. Histogramas para Ângulo e Intensidade da Polarização . . . . . . . . . . . . . . 115

A.1 Campos com Um Padrão . . . . . . . . . . . . . . . . . . . . . . 115

A.2 Campos com Dois Padrões . . . . . . . . . . . . . . . . . . . . 117

A.3 Campos com Nenhum Padrão . . . . . . . . . . . . . . . . . . . . . . . . . 119

A.4 Campos Filtrados por $m_{V} \ldots \ldots \ldots \ldots \ldots$

A.5 Campos com Estrelas Excluídas . . . . . . . . . . . . . . . . . . 121

A.6 Tabela com Parâmetros dos Padrões . . . . . . . . . . . . . . . . . . . . . 122

B. Mapas de Polarização . . . . . . . . . . . . . . . . . . . . . . . . . . . . . . . . 123

B.1 Mapas de Polarização por Campo . . . . . . . . . . . . . . . . . . . 123

B.2 Tabela com Objetos . . . . . . . . . . . . . . . . . . . . . 132 

Capítulo 1

\section{Introdução}

As Nuvens de Magalhães (NMs) são galáxias satélites da Via Láctea (VL), e juntamente com esta formam um sistema triplo em constante interação. Por serem galáxias próximas da VL e, mais do que isso, possuírem a vantagem de serem face-on, elas se tornam perfeitos laboratórios para estudos extragalácticos. As NMs são galáxias particularmente interessantes, pois, apesar da proximidade da Galáxia, elas apresentam metalicidade razoavelmente menor. Isso as torna ideais, dentre outras coisas, para entender a estrutura e evolução das primeiras galáxias. A Pequena Nuvem de Magalhães (PNM) se mostra ainda mais diferente da Galáxia do que a Grande Nuvem de Magalhães (GNM), motivo pelo qual ela é o foco deste trabalho.

A importância dinâmica do campo magnético em escalas galácticas vem sendo evidenciada cada vez mais, tanto pelo advento de simulações magneto-hidrodinâmicas quanto por medidas observacionais de alta resolução. Então, este trabalho teve como objetivo traçar a estrutura do campo magnético para a Pequena Nuvem de Magalhães, e entender a importância dele para esta galáxia.

A dissertação está organizada da seguinte maneira. Neste capítulo está apresentada a teoria em que este trabalho está fundamentado, e também é feita uma breve revisão bibliográfica sobre as Nuvens de Magalhães, em particular sobre a Pequena Nuvem de Magalhães. No capítulo 2 está descrita a técnica observacional utilizada para determinar a polarização interestelar no óptico, são apresentadas as características dos dados observados e como foi realizada a redução dos mesmos. No capítulo 3 são apresentados os resultados da redução dos dados e como foram determinadas a direção e a intensidade do campo magnético. No capítulo 4 são estudadas possíveis correlações entre polarização e 
outras componentes do meio interestelar (MI) da PNM e também é avaliada a importância dinâmica que o campo magnético possui para essa galáxia. No capítulo 5 são apresentadas as principais conclusões e perspectivas futuras para este trabalho.

\subsection{O Meio Interestelar}

O meio interestelar é composto por gás, poeira, raios cósmicos, um campo de radiação e campos magnéticos. Essas componentes estão altamente correlacionadas e vivem em equilíbrio, sendo uma das maiores evidências para este fato a equipartição aproximada entre as densidades de energia de seus constituintes (Maciel, 2002).

O campo de radiação é proveniente de diversas fontes e processos - estrelas, emissão térmica da poeira, resfriamento do gás, radiação cósmica de fundo etc. - e compreende todo o espectro eletromagnético, do rádio aos raios $\gamma$. Os raios cósmicos são partículas altamente energéticas cuja origem não é totalmente conhecida. Acredita-se que eles sejam produzidos em núcleos ativos de galáxias e explosões de supernova e em seguida acelerados até alcançar as altas energias observadas.

No meio interestelar também são observados campos magnéticos da ordem de alguns $\mu \mathrm{G}$. A sua existência foi inicialmente proposta por Fermi (1949) e confirmada observacionalmente para a Galáxia por Hiltner (1949) e Hall (1949). A origem de campos galácticos ainda não é conhecida com precisão, porém o mecanismo mais clássico utilizado para explicá-lo é o de um dínamo $\alpha$ - $\Omega$ (Ruzmaikin et al., 1988).

O gás e a poeira são os principais constituintes do meio interestelar. O gás que o compõe é observado em diferentes fases: meio frio neutro, morno neutro, morno parcialmente ionizado, morno ionizado e quente ionizado (Heiles, 2011). Essas cinco fases são associadas a diversas estruturas - nebulosas, nuvens moleculares, regiões HII etc. Já a poeira são grãos microscópicos compostos de carbono, silicatos e/ou grafites, e sempre é observada juntamente ao gás. Ela produz alguns efeitos observáveis como o avermelhamento da luz das estrelas e a polarização interestelar, sendo o último de particular interesse para este trabalho.

O meio interestelar está intimamente ligado à evolução das estrelas e das galáxias. Aquelas ejetam material para o MI na forma de ventos durante sua evolução. Esse material o enriquece com metais pesados produzidos nessas estrelas durante suas "vidas". Em 
seguida esse material vem a formar nuvens interestelares, as quais num momento posterior podem colapsar e formar novas estrelas, e desse modo o processo se repete continuamente. A poeira é gerada quando o material esfria o suficiente para que moléculas, e depois estruturas mais complexas, possam ser formadas. Também é necessário que a densidade do meio seja razoavelmente alta, assim as atmosferas e envelopes de estrelas gigantes e supergigantes frias são os locais mais prováveis para formação de grãos interestelares. O gás e a poeira interagem entre si e com as outras componentes do MI.

O campo de radiação (principalmente o estelar) e os raios cósmicos atuam, por exemplo, para o aquecimento e ionização do gás interestelar. Os campos magnéticos conseguem modificar o fluxo de gás nas galáxias; são importantes para explicar a formação de ventos e halos galácticos; possibilitam o afluxo de massa em núcleos ativos de galáxias; controlam a evolução de nuvens interestelares; e se mostram essenciais para o processo de formação estelar (Beck, 2004). Então, nota-se que o entendimento das componentes do meio interestelar é essencial para a compreensão da estrutura e da evolução das galáxias.

\subsection{Polarização da Luz}

Sejam duas ondas eletromagnéticas planas, monocromáticas e com a mesma frequência, uma com campo elétrico oscilando totalmente na direção $\vec{x}$ e a outra na direção $\vec{y}$. Pode-se considerar uma onda plana resultante propagando na direção $\vec{k}=k \vec{z}$, cuja representação matemática é dada por

$$
\vec{E}(\vec{r}, t)=\left(E_{o x} e^{i \delta_{x}} \vec{x}+E_{o y} e^{i \delta_{y}} \vec{y}\right) e^{i(\vec{k} \cdot \vec{r}-\omega t)}
$$

onde $\omega$ é a frequência da onda; $k$ é o número de onda; $E_{o x}$ e $E_{\text {oy }}$ são, respectivamente, as amplitudes da onda nas direções $\vec{x}$ e $\vec{y} ; \delta_{x}$ e $\delta_{y}$ são as fases relativas das componentes da onda.

Pode-se dizer que essa onda está polarizada para os seguintes casos:

(a) Se $\left|\delta_{x}-\delta_{y}\right|=2 n \pi$, com $n \in \mathbb{Z}$ : onda polarizada linearmente;

(b) Se $\left|\delta_{x}-\delta_{y}\right|=(2 n \pm 1) \pi$, com $n \in \mathbb{Z}$ : onda polarizada linearmente;

(c) Se $\left|\delta_{x}-\delta_{y}\right|=\left(2 n \pm \frac{1}{2}\right) \pi$, com $n \in \mathbb{Z}$ e $\left|E_{o x}\right|=\left|E_{\text {oy }}\right|$ : onda polarizada circularmente; 
(d) Outros casos: onda polarizada elipticamente.
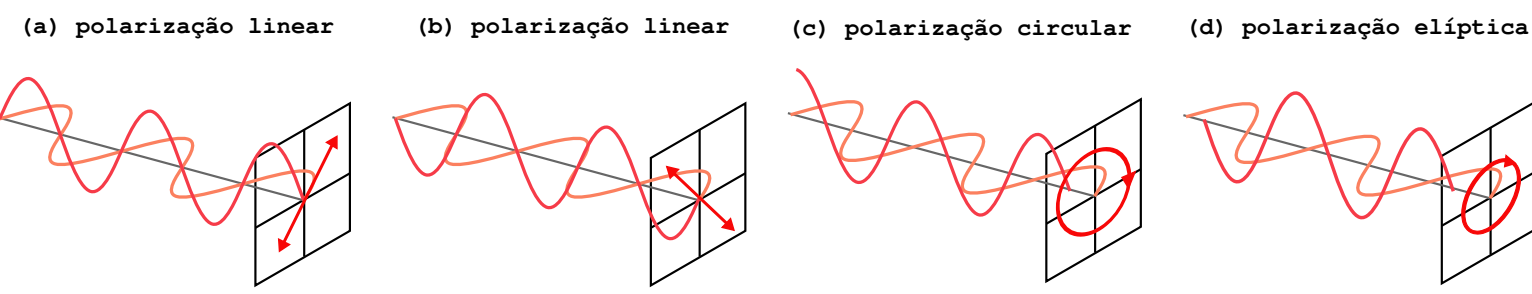

Figura 1.1: Representação dos estados (a), (b), (c) e (d) de polarização.

A figura 1.1 representa cada caso. Em (a), as componentes da onda estão em fase, ou seja, atingem seus máximos e mínimos ao mesmo tempo, e a onda está linearmente polarizada. Em (b), elas estão defasadas em $180^{\circ}$ e a onda é linearmente polarizada, porém com seu plano de vibração rotacionado com relação ao caso (a). Em (c), as componentes possuem magnitudes iguais e estão defasadas em $90^{\circ}$, o que causa a polarização circular. Dependendo do sentido em que o plano de vibração está rotacionando, diz-se que a onda está polarizada à direita ou à esquerda. Por fim, o caso (d) é o mais geral (Hecht, 2001).

Três processos físicos capazes de polarizar um feixe de luz são a reflexão, a absorção e o espalhamento. O primeiro acontece quando um feixe atinge uma superfície que absorve a maior parte das componentes da luz, refletindo apenas as que possuem um certo plano de vibração. O segundo, quando um feixe atravessa um material que absorve apenas certas componentes da luz. Já a polarização por espalhamento pode ser separada em três casos mais comuns - Rayleigh, Mie e Thomson. O espalhamento Rayleigh acontece quando a radiação interage com partículas e moléculas cujos tamanhos físicos sejam muito menores do que o comprimento de onda da radiação. O espalhamento Mie ocorre quando a radiação interage com partículas com tamanhos físicos da ordem do comprimento de onda da radiação. Por fim, o espalhamento Thomson acontece quando a radiação é espalhada por partículas carregadas e o limite de $h \nu /\left(m c^{2}\right)<<1$ é satisfeito.

Também existem mecanismos físicos que emitem luz intrinsecamente polarizada, exemplos são a radiação ciclotrônica e sincrotrônica. Em ambos os casos, partículas carregadas espiralando em torno de um campo magnético emitem radiação. A diferença entre eles é a velocidade com que a partícula está espiralando. No primeiro caso, ela possui velocidade muito menor do que a da luz. No segundo, ela é da ordem da velocidade da luz. A tabela 
1.1 apresenta alguns exemplos astrofísicos em que a polarização da luz pode ser observada, e os mecanismos físicos associados a eles. A figura 1.2 representa estes mecanismos.

Tabela 1.1 - Exemplos astrofísicos para diversos mecanismos de polarização da luz.

\begin{tabular}{ll}
\hline \hline Mecanismo & Exemplo $^{1}$ \\
\hline Reflexão & Superfícies sólidas de planetas e asteroides \\
Espalhamento Rayleigh & Moléculas em atmosferas de estrelas frias e planetas \\
Espalhamento Mie & Poeira interestelar, envelopes de estrelas, nebulosas de reflexão e luz zodiacal \\
Espalhamento Thomson & Elétrons livres em envelopes de estrelas e radiação cósmica de fundo \\
Radiação ciclotrônica & Elétrons espiralando em campos magnéticos de anãs brancas \\
Radiação sincrotrônica & Elétrons espiralando em campos magnéticos de supernovas, radiogaláxias e quasares
\end{tabular}
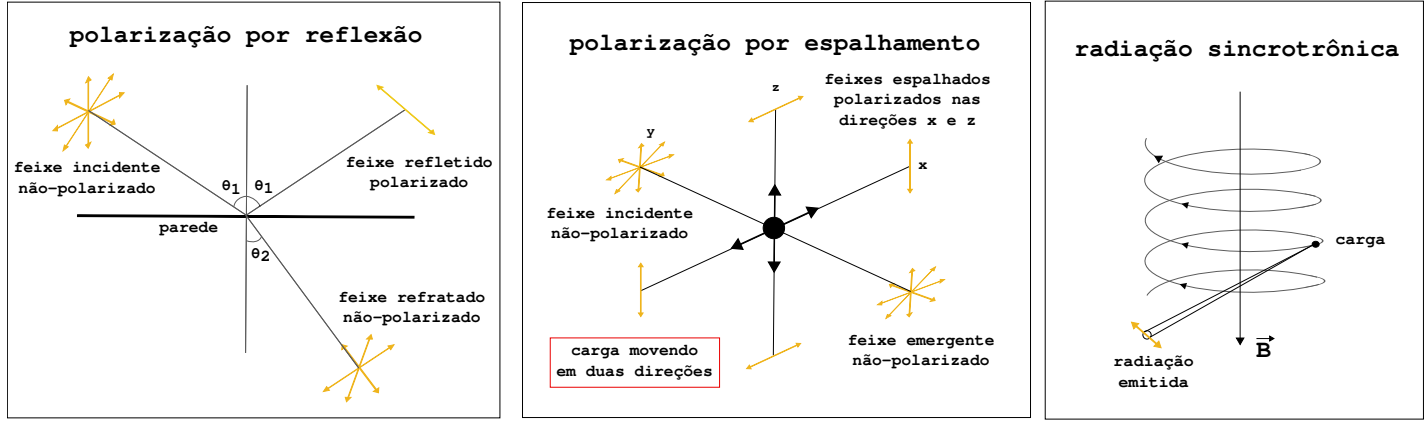

Figura 1.2: Mecanismos físicos capazes de polarizar um feixe de luz e mecanismo que emite luz intrinsecamente polarizada.

\subsubsection{Os Parâmetros de Stokes}

Uma forma de representar o estado de polarização da luz é através dos parâmetros de Stokes, os quais foram definidos por G. G. Stokes em 1852. Supondo a onda plana da equação 1.1, a definição matemática deles é expressa por

$$
\left\{\begin{array}{l}
I=S_{0}=\left\langle E_{o x}^{2}\right\rangle+\left\langle E_{o y}^{2}\right\rangle, \\
Q=S_{1}=\left\langle E_{o x}^{2}\right\rangle-\left\langle E_{o y}^{2}\right\rangle, \\
U=S_{2}=2\left\langle E_{o x} E_{o y} \cos \left(\delta_{x}-\delta_{y}\right)\right\rangle, \\
V=S_{3}=2\left\langle E_{o x} E_{o y} \operatorname{sen}\left(\delta_{x}-\delta_{y}\right)\right\rangle,
\end{array}\right.
$$

\footnotetext{
${ }^{1}$ Exemplos retirados das notas de aula de Magalhães.
} 
onde $I$ representa a intensidade total do feixe; $Q$ e $U$ os parâmetros que definem polarização linear; e $V$ o que define polarização circular. Eles estão ligados pela desigualdade $I^{2} \geq Q^{2}+U^{2}+V^{2}$, a qual ocorre devido ao fato de a luz não precisar ser totalmente polarizada. As médias no tempo são necessárias, pois pode haver alguma variação das grandezas (Jackson, 1998).

Do ponto de vista observacional, a forma mais útil de representar os parâmetros de Stokes é operacionalmente. Supondo filtros que transmitam metade da luz incidente, podemos arranjá-los de forma que seja possível a determinação dos parâmetros de Stokes através da intensidade da luz que cada um deixa passar (Hecht, 2001). A figura 1.3 representa uma possível combinação de filtros. O filtro $I_{1}$ permite, apenas, a passagem da componente polarizada na direção 0 graus; $I_{2}$ da componente polarizada na direção 45 graus; $I_{3}$ da componente polarizada à esquerda; $I_{4}$ da componente polarizada a 90 graus; $I_{5}$ da componente polarizada a 135 graus; e $I_{6}$ da componente polarizada à direita.

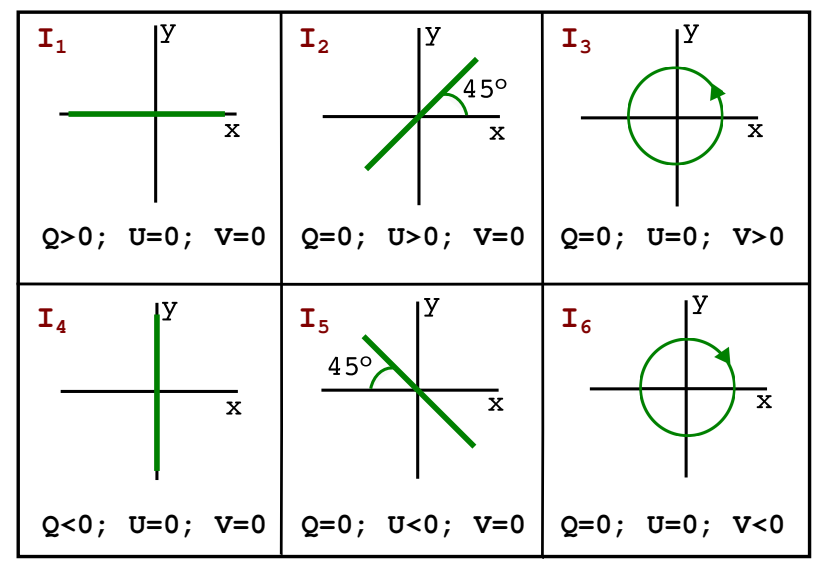

Figura 1.3: Desenho esquemático de filtros que podem ser utilizados para determinar os parâmetros de Stokes. Figura cedida gentilmente por Nadili Louise Ribeiro.

Utilizando estes filtros, a definição operacional dos parâmetros de Stokes é representada por

$$
\left\{\begin{array}{l}
I=I_{0}, \\
Q=\left(2 I_{1}-I_{0}\right)=-\left(2 I_{4}-I_{0}\right)=\left(I_{1}-I_{4}\right), \\
U=\left(2 I_{2}-I_{0}\right)=-\left(2 I_{5}-I_{0}\right)=\left(I_{2}-I_{5}\right), \\
V=\left(2 I_{3}-I_{0}\right)=-\left(2 I_{6}-I_{0}\right)=\left(I_{3}-I_{6}\right),
\end{array}\right.
$$

onde $I_{0}$ é a intensidade total do feixe, que pode ser obtida na ausência de filtros; e $I_{1}$ a $I_{6}$ 
são as intensidades que passam pelos filtros 1 a 6 , respectivamente. Assim, o parâmetro $Q$, que define polarização linear, pode ser obtido pela diferença das intensidades que emergem dos filtros 1 e 4; o parâmetro $U$, que também define polarização linear, pela diferença das intensidades que emergem dos filtros 2 e 5; e o parâmetro $V$, que define polarização circular, pela diferença das intensidades que emergem dos filtros 3 e 6 .

Conhecendo-se os parâmetros de Stokes, a polarização linear de um feixe de luz é dada por

$$
\left\{\begin{array}{l}
P=\sqrt{Q^{2}+U^{2}}, \\
\theta=\frac{1}{2} \operatorname{arctg}\left(\frac{U}{Q}\right),
\end{array}\right.
$$

onde $P$ é o grau de polarização; e $\theta$ é o ângulo de polarização. Para determinar a polarização circular, basta dividir o parâmetro $V$ pela intensidade total $I$.

Uma propriedade interessante e útil dos parâmetros de Stokes é que eles são aditivos. De modo que se diferentes feixes de luz são sobrepostos, os parâmetros de Stokes do feixe resultante são a soma dos parâmetros de cada feixe individual. É importante destacar também que a direção $\theta=0$ graus, eixo $x$ na figura 1.3 , corresponde a direção Norte na Astronomia.

\subsubsection{Polarização pelo Meio Interestelar}

Como foi mencionado na seção 1.1, a polarização interestelar é um dos efeitos observáveis causado pela presença de grãos de poeira no MI. A luz emitida pelas estrelas em geral é não-polarizada, ou seja, o vetor campo elétrico não possui direção preferencial de vibração. Porém, ao atravessar o MI, essa radiação interage com os grãos de poeira presentes nele, e ganhando com isso um certo grau de polarização.

A primeira implicação da polarização linear observada é a de que esses grãos de poeira não podem ser esféricos e isotrópicos. Desse modo, ao atravessá-los, a luz continuaria sendo não-polarizada, já que seria absorvida e reemitida em todas as direções.

A figura 1.4 ilustra como a polarização é causada. Supondo que os grãos de poeira possuam uma forma alongada, a luz ao atravessá-lo será mais absorvida na direção do eixo maior do grão, sendo as outras componentes espalhadas. A luz espalhada é polarizada na direção de rotação do grão. E a luz absorvida age no sentido de aquecer o grão, e depois é reemitida como radiação de corpo negro, com pico no infravermelho distante. 
Essa radiação de corpo negro é polarizada na direção perpendicular ao eixo de rotação do grão.

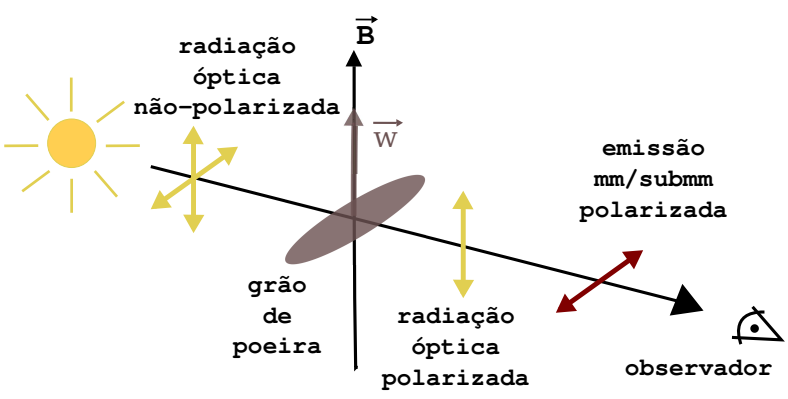

Figura 1.4: Ilustração de como a radiação óptica não-polarizada emitida pelas estrelas se torna linearmente polarizada, ao atravessar grãos de poeira no meio interestelar, e de como a radiação infravermelha emitida pelos grãos pode ser intrinsecamente polarizada. Figura cedida gentilmente por Nadili Louise Ribeiro.

Apesar de supor que estes grãos interestelares sejam alongados, seria de se esperar que a distribuição espacial de seus eixos de rotação fosse aleatória. Assim, o efeito líquido da polarização por absorção dos diversos grãos destruiria a polarização causada por um único grão. Então, além de ser necessário que os grãos sejam alongados, é também essencial algum mecanismo fazer com que pelo menos parte dos grãos possuam seus eixos de rotação orientados numa direção preferencial.

O mecanismo de Davis e Greenstein (1951) foi o primeiro a obter algum sucesso ao tentar explicar esse alinhamento. Ele supõe grãos paramagnéticos, de modo que um campo magnético externo induz um momento magnético nos grãos. As diversas colisões entre os grãos de poeira e o gás acarreta a aquisição de rotação pelos grãos, existindo equipartição entre a energia média de rotação dos grãos e a térmica do gás.

O movimento de rotação da poeira leva a uma constante mudança na direção do momento magnético em função do tempo. Acontece que tal mudança provoca perdas na energia de rotação do grão. Portanto, esse mecanismo supõe que exista dissipação paramagnética. Um torque aplicado no grão age no sentido de tornar o campo magnético e o eixo de rotação dos grãos alinhados, de modo a não existir mais perdas de energia. Pois, nesse caso, a direção do momento magnético deixa de variar com o tempo, resultando então no alinhamento requerido para explicar a polarização observada. Nota-se que o mecanismo também supõe a existência de um campo magnético externo. Isso não foi um problema, já 
que Hiltner e Hall, em 1949, observaram polarização interestelar para a Galáxia, indicando que este campo externo realmente deveria existir.

No mecanismo de Davis e Greenstein, o problema é que o tempo de relaxação paramagnética é muito alto. Assim, a alta taxa de colisões entre os grãos e o gás não deixaria que o torque levasse ao alinhamento dos grãos com o campo magnético. Hoje em dia, o mecanismo mais aceito para explicar tal alinhamento é o de torques radiativos. A interação entre um grão de poeira e a componente anisotrópica do campo de radiação estelar provoca o chamado torque radiativo.

Hoang e Lazarian (2009) mostraram que o torque radiativo, sofrido por grãos com formato helicoidal, primeiramente alinha o eixo menor do grão com seu momento angular, e posteriormente estes dois eixos com o campo magnético. O problema é que para isso ocorrer a energia de rotação do grão deve ser maior do que a energia térmica do gás. Porém, Lazarian e Hoang (2008) mostraram que grãos superparamagnéticos sempre podem satisfazer tal condição. A necessidade de que os grãos sejam helicoidais e superparamagnéticos dificulta a explicação para o alinhamento dos grãos em ambientes onde este tipo de poeira é improvável. Então, apesar de possuir vários avanços, a teoria do alinhamento de grãos ainda não é completamente compreendida.

\subsection{O Método de Chandrasekhar \& Fermi}

Existem diversas técnicas observacionais que podem ser utilizadas para estudar campos magnéticos cósmicos - emissão síncrotron, efeito Zeeman, rotação Faraday e polarização interestelar. O método de Chandrasekhar e Fermi (1953) é um procedimento muito simples que pode ser empregado para determinar a intensidade do campo magnético projetada no plano do céu, valendo-se da polarização interestelar. Ele é baseado na dispersão dos vetores de polarização de um conjunto de estrelas.

Lembrando que a polarização interestelar é aceita como fruto do alinhamento dos grãos de poeira com um campo magnético galáctico, nota-se que, quando medimos os vetores de polarização no óptico de um conjunto de estrelas, estamos mapeando também o campo magnético projetado no plano do céu $\left(\vec{B}_{\text {céu }}\right)$. Desse modo, eles imaginaram que a dispersão na direção dos vetores de polarização deveria ter alguma relação com o campo magnético e com o movimento de turbulência do gás. A teoria de que existe um "congelamento" do 
campo magnético com relação ao gás é bem aceita, ou seja, $\vec{B}$ é carregado pelo gás. Assim, qualquer tipo de turbulência nele deve levar a algum encurvamento nas linhas de campo, e esse encurvamento deve estar relacionado à dispersão dos vetores de polarização.

Sabe-se que pode ocorrer o aparecimento de ondas em fluidos submetidos a pequenas perturbações. Alfvén (1942) mostrou, no caso de fluidos magnetizados, poderem surgir modos que mais tarde ficaram conhecidos como ondas Alfvén. Estas se tratam de uma flutuação de velocidade transversal, tanto a $\vec{k}$ (direção de propagação da perturbação) quanto a $\vec{B}$. Por ser perpendicular ao campo, essa flutuação faz com que as linhas se encurvem. A velocidade de fase deste modo é expressa por

$$
v_{A}=\frac{B}{\sqrt{4 \pi \rho}}
$$

onde $B$ é o campo magnético; e $\rho$ é a densidade da matéria difusa, já que as estrelas não devem interagir de modo significativo com o campo.

Como esse tipo de perturbação pode ser caracterizado por exponenciais imaginárias, a oscilação transversal de uma linha de campo particular pode ser representada por

$$
y=A \cos \left(k x-k v_{A} t\right)
$$

onde $A$ é a amplitude da oscilação; $k$ é o número de onda associado à perturbação; $y$ é o deslocamento lateral da linha de campo; e $x$ é a coordenada paralela à linha de campo.

Então, para descobrir a velocidade lateral com que uma linha de campo se move e o quanto esse deslocamento varia ao longo de $x$, pode-se calcular, respectivamente, as derivadas parciais com relação a $t$ e a $x$

$$
\left\{\begin{array}{l}
\frac{\partial y}{\partial t}=\dot{y}=A k v_{A} \operatorname{sen}\left(k x-k v_{A} t\right) \\
\frac{\partial y}{\partial x}=y^{\prime}=-A k \operatorname{sen}\left(k x-k v_{A} t\right) .
\end{array}\right.
$$

Tomando a média quadrática das duas equações acima e dividindo uma pela outra temos que

$$
\overline{\dot{y}}^{2}=v_{A}^{2} \bar{y}^{\prime 2}
$$

O maior "truque" desse método foi perceber que a dispersão dos vetores de polarização $(\delta \theta)$ pode ser associada à variação média do deslocamento lateral das linhas de campo ao longo dele, ou seja, $\delta \theta^{2}={\overline{y^{\prime}}}^{2}$. 
Outra consideração feita é relacionada à velocidade lateral média com que as linhas de campo se movem. Essa velocidade lateral nada mais é do que a velocidade de turbulência com que o gás se move na direção perpendicular às linhas de campo, no caso a dispersão de velocidades na linha de visada $\left(\delta V_{\mathrm{LOS}}{ }^{2}\right)$, pois a informação que temos é com relação a $\vec{B}_{\text {céu }}$, assim $\overline{\dot{y}}^{2}=\delta V_{\text {LOS }}^{2}{ }^{3}$.

Utilizando essas associações e combinando as equações 1.12 e 1.16, temos que

$$
B_{\text {céu }}=\sqrt{4 \pi \rho} \frac{\delta V_{\mathrm{LOS}}}{\delta \theta} .
$$

A equação 1.17 resume o método de Chandrasekhar e Fermi (método CF). Nota-se que ela está em completa concordância com o raciocínio original. Se $\delta \theta$ é alto, essa equação leva a um valor baixo para a intensidade de $B_{\text {céu }}$, o que é de se esperar, já que, se as linhas de campo estão se encurvando muito, isso deve levar a uma diminuição na magnitude do campo. Isso também significa que a turbulência no meio é alta. Por outro lado, o campo é alto para o caso em que $\delta \theta$ é pequeno, ou seja, a turbulência é baixa e existe pouco encurvamento das linhas de campo.

\subsubsection{Método CF Modificado}

A equação 1.17 é válida apenas para casos em que $\delta \theta$ é pequeno. Quando foi feita a associação de $\overline{y^{\prime}}=\delta \theta$, no fundo também foi utilizada a aproximação de que $\delta \theta \sim \delta(\operatorname{tg} \theta)$ (ver figura 1.5). Heitsch et al. (2001) realizaram a troca $\delta \theta \rightarrow \delta(\operatorname{tg} \theta)$ no método $\mathrm{CF}$, porém perceberam que esta levava a subestimar os valores do campo em larga escala quando o fluido era superalfvénico ${ }^{4}$, caso em que o campo turbulento pode ser alto. Para evitar esse problema eles introduziram uma correção: calculavam a média geométrica entre os campos magnéticos obtidos pelo método CF padrão e o modificado por eles. Porém, nota-se que essa correção é mais um artifício matemático do que uma solução física.

Dentre as diversas correções propostas para o método CF, uma que parece promissora e conseguiu obter resultados satisfatórios para a intensidade do campo para diversos tipos de fluidos foi proposta por Falceta-Gonçalves et al. (2008). Eles notaram que uma forma

\footnotetext{
${ }^{2}$ LOS $=$ line of sight (linha de visada).

${ }^{3}$ Originalmente Chandrasekhar e Fermi (1953) partiram da hipótese de que a velocidade de turbulência é isotrópica, e então utilizaram $\overline{\dot{y}}^{2}=\frac{1}{3} v^{2}$, onde $v$ é a velocidade de turbulência do fluido.

${ }^{4}$ Um fluido é superalfvénico quando sua velocidade média é maior do que a velocidade Alfvén.
} 


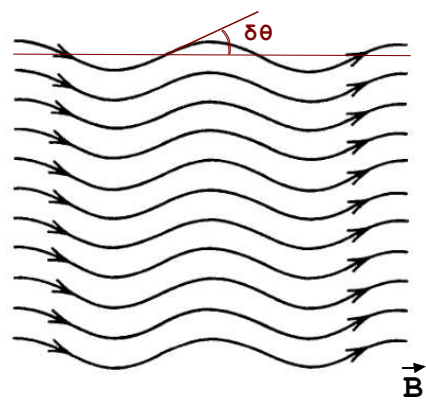

Figura 1.5: Encurvamento das linhas de campo devido ao movimento de turbulência do gás. Adaptada de Jackson (1998).

alternativa de deduzir o método CF é partindo diretamente da suposição de equipartição entre as densidades de energias turbulentas magnética e cinética do gás

$$
\frac{1}{2} \rho \delta V_{\mathrm{LOS}}^{2} \simeq \frac{1}{8 \pi} \delta B^{2}
$$

onde $\delta B$ é a componente turbulenta do campo magnético. A equação acima assume que tanto o campo magnético turbulento quanto o movimento de turbulência do gás são isotrópicos. Essa abordagem é equivalente à suposição de Chandrasekhar e Fermi (1953) de o encurvamento das linhas de campo de $\vec{B}$ estarem associados à turbulência do gás. A aproximação para ângulos pequenos também pode ser escrita como $\delta \theta \sim \delta B / B_{\text {céu }}$ (Zweibel, 1990). Então, substituindo-a na equação 1.18 retomamos a equação 1.17.

Para generalizar essa equação para quando a aproximação de ângulos pequenos não for boa, pode-se aplicar duas correções simples à equação 1.18. A primeira é a de o campo magnético total no plano do céu ser expresso por $B_{\text {céu }}^{\text {tot }}=B_{\text {céu }}+\delta B$, o que novamente supõe o campo turbulento ser isotrópico. A segunda é de que $\operatorname{tg}(\delta \theta) \sim \delta B / B_{\text {céu }}^{\text {tot }}$, assumindo $\delta B / B_{\text {céu }}^{\text {tot }}$ ser global. E então não existe problema em primeiro olhar a dispersão de $\theta$ e depois calcular sua tangente. Assim, substituindo essas correções na equação 1.18, temos

$$
B_{\text {céu }}+\delta B \simeq \sqrt{4 \pi \rho} \frac{\delta V_{\mathrm{LOS}}}{\operatorname{tg}(\delta \theta)} .
$$

A equação 1.19 resume o método CF modificado por Falceta-Gonçalves et al. (2008). Nota-se a partir dela e da aproximação de $\delta B / B_{\text {céu }}^{\text {tot }}$ que para $\delta \theta \rightarrow 0, \delta B \rightarrow 0$, o que já era esperado desde o início; para $\delta \theta \rightarrow \pi / 4$, temos $B_{\text {céu }}^{\text {tot }} \rightarrow \delta B$, o que deve acontecer para o caso no qual o campo tem uma inclinação muito grande com relação ao plano do céu (ou seja, sua componente nele é próxima de zero, restando apenas a componente turbulenta), 
ou quando simplesmente o fluido for superalfvénico, o que indica $\delta B$ alto, podendo até suprimir o campo em larga escala; e para $\delta \theta \rightarrow \pi / 2$, temos $B_{\text {céu }} \rightarrow \delta B$, onde o campo magnético em larga escala é da ordem do campo turbulento.

\subsection{Nuvens de Magalhães}

A Pequena e a Grande Nuvem de Magalhães são galáxias irregulares ricas em gás e que orbitam a Via Láctea (VL), formando um sistema triplo em constante interação. Devido à proximidade com a Galáxia e à alta latitude galáctica, as Nuvens de Magalhães se tornam um ótimo laboratório para vários tipos de estudos. É possível realizar medidas observacionais com alta resolução de diversas estruturas, já que a luz emitida por elas não é muito atenuada por causa da poeira presente no disco Galáctico.

As NMs são galáxias com baixa metalicidade $-0,5 Z_{\odot}^{5}$ e $0,2 Z_{\odot}$ para a GNM e a PNM, respectivamente (Kurt e Dufour, 1998). Devido a isso, o meio interestelar dessas galáxias possui propriedades particularmente diferentes das observadas para a VL. Dentre as maiores diferenças, podemos destacar a alta razão gás/poeira (Meixner et al., 2010; Bot et al., 2004; Stanimirović et al., 2000) e o excesso de emissão no submm (Planck Collaboration, 2011; Bot et al., 2010; Israel et al., 2010; Bernard et al., 2008). O conteúdo do MI das NMs também indica que elas são galáxias relativamente jovens.

As Nuvens de Magalhães são objetos altamente estudados. A maior motivação para tais estudos consiste no fato de que o entendimento delas é fundamental para se compreender, por exemplo, a evolução de galáxias de altos redshifts até hoje em dia; a estrutura de anãs irregulares e do conteúdo do MI das partes externas de galáxias espirais grandes; a formação estelar em ambientes de baixa metalicidade; galáxias anãs em interação; e a relação de galáxias satélites com as suas hospedeiras.

\subsubsection{A Pequena Nuvem de Magalhães}

A Pequena Nuvem de Magalhães possui um MI mais diferente da VL do que a GNM, no entanto ela em geral é menos estudada. Esses são dois dos motivos que encorajou este trabalho a se focar na PNM.

\footnotetext{
${ }^{5} Z_{\odot}$ é a metalicidade do Sol, comumente utilizada como referência para metalicidade de outros objetos.
} 
Esta galáxia possui um diâmetro angular no céu de aproximadamente $5^{\circ}$, e de acordo com medidas de magnitudes aparentes de estrelas gigantes se encontra a uma distância de $(63 \pm 1)$ kpc (Cioni et al., 2000). As duas estruturas em larga escala da PNM que mais se destacam são sua Barra e sua Asa (ver figura 1.6). Abaixo está apresentada uma breve revisão bibliográfica do que se conhece sobre o MI desta galáxia.

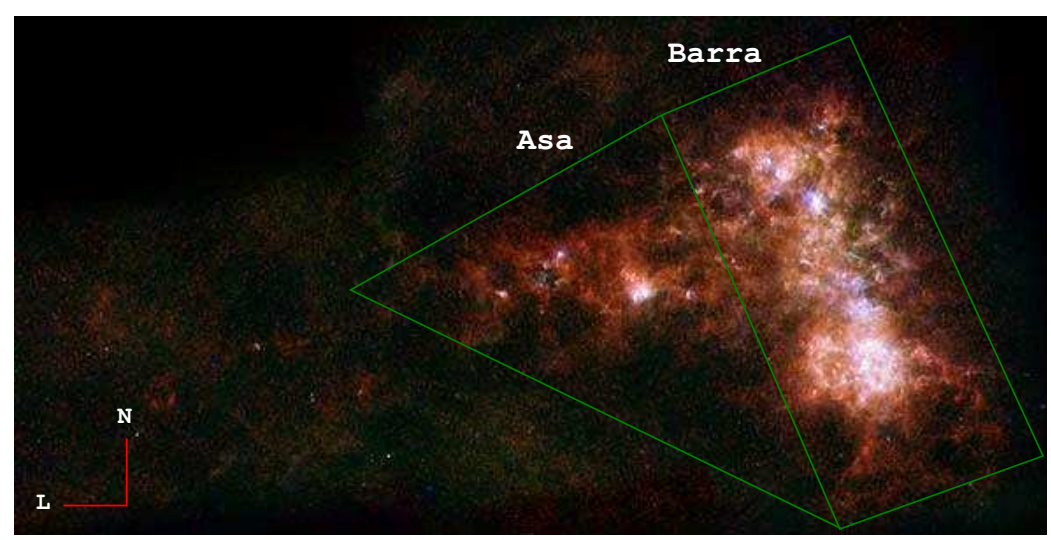

Figura 1.6: Imagem composta da PNM - Herschel-SPIRE, Herschel-PACS e Spitzer-MIPS - $250 \mu \mathrm{m}$ (vermelho); 100 e $160 \mu \mathrm{m}$ (verde); e 24 e $70 \mu \mathrm{m}$ (azul).

\subsubsection{Radiação Ultravioleta}

O campo de radiação no ultravioleta para a PNM é cerca de 4-10 vezes maior do que o da vizinhança solar (Vangioni-Flam et al., 1980). Essa emissão no UV é dada principalmente por estrelas do tipo $\mathrm{O}$ e B, indicando então que a PNM possui alta população estelar jovem. Observações recentes no UV-distante, feitas pelo satélite Far Ultraviolet Spectroscopic Explorer (FUSE), também mostraram que a emissão nessa região do espectro está fortemente correlacionada com a emissão H $\alpha$ de regiões HII (Pradhan et al., 2011), correlação esta já esperada por o campo de radiação estelar ser aquele que geralmente aquece o gás formador das regiões HII.

\subsubsection{Campo Magnético}

O campo magnético na PNM já foi previamente estudado através da aplicação de diversas técnicas - polarização interestelar no óptico, rotação Faraday e emissão síncrotron.

Magalhães et al. (2009) reanalisaram dados fotoelétricos de polarização interestelar no óptico, apresentados em Magalhães et al. (1990), e obtiveram um campo magnético em 
larga escala projetado no plano do céu da ordem de $1,7 \mu \mathrm{G}$ e um campo turbulento de 3,5 $\mu \mathrm{G}$. Mao et al. (2008) utilizaram medidas fotoelétricas para polarização interestelar no óptico de Schmidt $(1976,1970)$ e Mathewson e Ford (1970b,a) e obtiveram um campo magnético projetado no plano do céu de $B_{\text {céu }}=(1,6 \pm 0,4) \mu \mathrm{G}$.

Mao et al. (2008) também estimaram o campo magnético na direção da linha de visada, através da rotação Faraday, com dados provindos do Australia Telescope Compact Array (ATCA). Estimaram uma intensidade de $B_{\mathrm{LOS}}=(0,19 \pm 0,06) \mu \mathrm{G}$ e que o campo está apontando na direção contrária a nós.

Existem também diversos estudos baseados na emissão do contínuo do rádio. A partir da emissão síncrotron, e supondo equipartição entre as energias dos raios cósmicos e do campo magnético, Loiseau et al. (1987) estimaram um campo magnético para a PNM da ordem de $5 \mu \mathrm{G}$.

\subsubsection{Hidrogênio Ionizado}

O meio ionizado da PNM já foi mapeado a partir da observação da emissão $\mathrm{H} \alpha$. O primeiro levantamento foi feito por Henize (1956), que detectou diversas nebulosas de emissão. Posteriormente, Davies et al. (1976) fizeram um levantamento mais completo, e identificaram quais dessas nebulosas podiam ser classificadas como regiões HII. Existem também medidas mais recentes feitas pelo Southern H-Alpha Sky Survey Atlas (SHASSA) (Gaustad et al., 2001), que cobriu toda a PNM. Uma grande parte dessa emissão está associada a regiões HII, comumente conhecidas como berçário de estrelas.

\subsubsection{Hidrogênio Neutro}

O levantamento mais recente e mais completo que se tem sobre o hidrogênio neutro (HI) na PNM foi feito por Stanimirović et al. (1999). Eles combinaram dados dos telescópios Parkes e ATCA, o que possibilitou o conhecimento da distribuição espacial do HI para escalas entre 30 pc e 4 kpc. Também realizaram a construção do espectro de potência espacial do HI, e observaram que ele obedece razoavelmente bem a relação $P(k) \propto k^{-3,04 \pm 0,02}$. E essa potência é compatível com o valor obtido para a Galáxia, demonstrando os MIs de diferentes galáxias poderem ter um mesmo mecanismo de formação de estruturas. A interpretação que eles deram para esse espectro de potência é de que o MI da PNM tem na- 
tureza fractal e consiste de uma hierarquia de nuvens de HI as quais podem ter se formado, por exemplo, por turbulência homogênea. O estudo da cinemática do HI possibilitou a deteç̧ão de três "camadas gigantes" (supergiants shells) que possuem diâmetros de até 1,8 kpc, e parecem ser as mesmas previamente identificadas por Hindman (1967).

Estudos mais detalhados da cinemática do HI, feitos por Stanimirović et al. (2004), mostraram existir um grande gradiente de velocidades para o HI. O mapa do campo de velocidades sugeriu haver rotação diferencial, e a construção da sua curva de rotação mostrou que ela possui um pico para $v_{\text {rot }}=60 \mathrm{~km} / \mathrm{s}$, alcançado para um raio de apenas $\sim 3 \mathrm{kpc}$.

\subsubsection{Gás Molecular}

O gás molecular da PNM foi mapeado pela primeira vez por Rubio et al. (1991), que utilizaram dados obtidos pelo Columbia $1.2 \mathrm{~m}$ Millimeter-Wave Telescope para transição rotacional do ${ }^{12} \mathrm{CO}(\mathrm{J}=1 \rightarrow 0)$. Mais tarde, Mizuno et al. (2001) realizaram medidas mais precisas utilizando o NANTEN Millimeter-Wave Telescope. Ambos notaram que a maior parte da emissão de CO se concentra nas regiões Sudoeste e Nordeste da Barra da PNM, e está fortemente associada à formação estelar recente. Foram obtidas temperaturas de antena para as nuvens densas de CO menores do que as observadas na Galáxia, indicando que elas possuem tamanhos substancialmente menores.

O fator de conversão para a emissão de $\mathrm{CO}$ em densidade de coluna de $\mathrm{H}_{2}$ obtido é relativamente maior do que o da Galáxia ( $~ 10$ vezes), indicando que a PNM possui fraca emissão daquela molécula. A interpretação que Mizuno et al. (2001) deram é de que essa diferença pode ser consequência da baixa abundância dos átomos de $\mathrm{C}$ e $\mathrm{O}$. A baixa abundância de poeira também pode possibilitar que a radiação UV penetre as partes mais internas das nuvens moleculares, o que causa uma maior fotodissociação do CO. Já a molécula $\mathrm{H}_{2}$ consegue sobreviver até raios maiores. Assim, o $\mathrm{CO}$ não se mostra um bom traçador para essa espécie, podendo levar à subestimação da sua massa.

Posteriormente, Bot et al. (2007) estudaram a abundância do gás molecular utilizando a emissão da poeira no submm como traçadora. Foram feitas observações com o Swedish ESO Submilimeter Telescope (SEST), utilizando o instrumento SEST Imaging Bolometer Array (SIMBA). As massas derivadas para as nuvens moleculares foram sistematicamente maiores (da ordem de 2 vezes) do que as obtidas empregando o teorema do Virial e observações da 
emissão de CO. Porém, essa diferença não pode ser explicada por nenhuma mudança nas propriedades ópticas da poeira na PNM. Ela parece ser devida à pressão magnética que ajuda no suporte das nuvens.

\subsubsection{Poeira}

A poeira presente no MI da PNM também é altamente estudada. O primeiro mapeamento completo no infravermelho (IV) distante foi feito pelo satélite Infrared Astronomical Satellite (IRAS), nos comprimentos de onda de 12, 25, 60 e $100 \mu \mathrm{m}$. Stanimirović et al. (2000) estudaram os dados do IRAS, e a partir da construção do espectro integrado perceberam que a emissão no IV-distante não podia ser devida a grãos muito pequenos ou moléculas $P A H s^{6}$. Eles estimaram que a temperatura dos grãos deve estar no intervalo entre 23-45 K, ou seja, os grãos na PNM são em geral mais quentes do que os da Galáxia, os quais possuem temperaturas entre $17-30 \mathrm{~K}$.

A razão gás/poeira média obtida foi 30 vezes maior do que o valor para a Galáxia. O espectro de potência espacial para a emissão dos grãos em 60 e $100 \mu$ m obedece à relação $P(k) \propto k^{-3,2 \pm 0,1}$, compatível com a lei de potência obtida para os dados de HI. Isso indica que as nuvens de poeira possuem o mesmo comportamento que as de HI. O espectro de coerência espacial para a poeira e o HI mostrou ambos estarem bem correlacionados para escalas maiores do que 50 pc, com o coeficiente de correlação maior do que $60 \%$.

Bot et al. (2004) também estudaram a emissão dos grãos no IV-distante somando aos dados do IRAS outros obtidos para $170 \mu \mathrm{m}$, com o satélite Infrared Space Observatory (ISO) utilizando o instrumento ISO Imaging Photopolarimeter (ISOPHOT). A razão gás/poeira obtida com esse estudo foi a mesma que a obtida por Stanimirović et al. (2000), que é maior por um fator 3 do que o esperado pela diferença em metalicidade com a Galáxia. Eles obtiveram uma temperatura de equilíbrio para os grãos no meio difuso de (22 \pm 2$) \mathrm{K}$. O modelo empregado para ajustar a distribuição espectral de energia não favoreceu moléculas PAHs, grãos muito pequenos e nem muito grandes, e também apresentou um excesso de emissão para $60 \mu \mathrm{m}$ que eles não conseguiram explicar.

Planck Collaboration (2011) estudou o excesso no submm apresentado na distribuição espectral de energia, somando dados dos satélites IRAS, Wilkinson Microwave Anisotropy

\footnotetext{
${ }^{6} \mathrm{PAHs}=$ polycyclic aromatic hydrocarbons (hidrocarbonetos aromáticos policíclicos).
} 
Probe (WMAP) e Planck. Eles tentaram explicar o excesso de emissão no submm a partir de flutuações da radiação cósmica de fundo, e obtiveram sucesso para o caso da GNM (que também apresentava esse excesso), porém não para a PNM. Eles sugeriram que talvez o excesso seja devido a grãos grandes de natureza amórfica somados a grãos com movimento de rotação rápida.

\section{Curva de Extinção}

As curvas de extinção conhecidas para a PNM, diferentemente da Galáxia, possuem um aumento acentuado para o UV-distante, e em geral não apresentam a "corcova" para $2175 \AA$ A. Uma possível explicação é que a abundância de carbono na forma sólida é baixa ou inexistente ou que a distribuição de tamanho dos grãos é diferente da obtida para a VL (Rodrigues et al., 1997).

Gordon et al. (2003) estudaram todas as curvas de extinção conhecidas para a PNM. Eles atribuíram as diferenças apresentadas entre as curvas da PNM e as da Galáxia como fruto da amostra de estrelas que foram utilizadas para a construção delas. Diferente do caso da VL, para a PNM as estrelas estão sempre associadas a regiões ativas e possuem um viés para estrelas OB supergigantes.

Eles dividiram as curvas de extinção da PNM em dois grupos, um com a curva de extinção média para a Barra da PNM, construída utilizando uma amostra de 4 estrelas (AzV 18, AzV 23, AzV 214 e AzV 398). O outro com a curva para a Asa da PNM, que foi construída utilizando apenas a estrela $\mathrm{AzV}$ 456, única que possui a "corcova" para $2175 \AA$ e não apresenta aumento acentuado para o UV. As curvas médias obtidas estão apresentadas abaixo e os seus gráficos na figura 1.7.

\section{- Barra da PNM:}

$$
\left\{\begin{array}{l}
A_{V}=(2,74 \pm 0,13) E(B-V), \\
N(H I)=(13,18 \pm 1,02) \times 10^{21} A_{V} .
\end{array}\right.
$$

- Asa da PNM:

$$
\left\{\begin{array}{l}
A_{V}=(2,05 \pm 0,17) E(B-V) \\
N(H I)=(7,40 \pm 1,20) \times 10^{21} A_{V}
\end{array}\right.
$$


Onde $A_{V}$ é a extinção na banda $\mathrm{V}$ medida em mag; $E(B-V)$ é o excesso de cor medido em mag; e $N(H I)$ é a densidade de coluna do HI medida em átomos.cm ${ }^{-2}$.
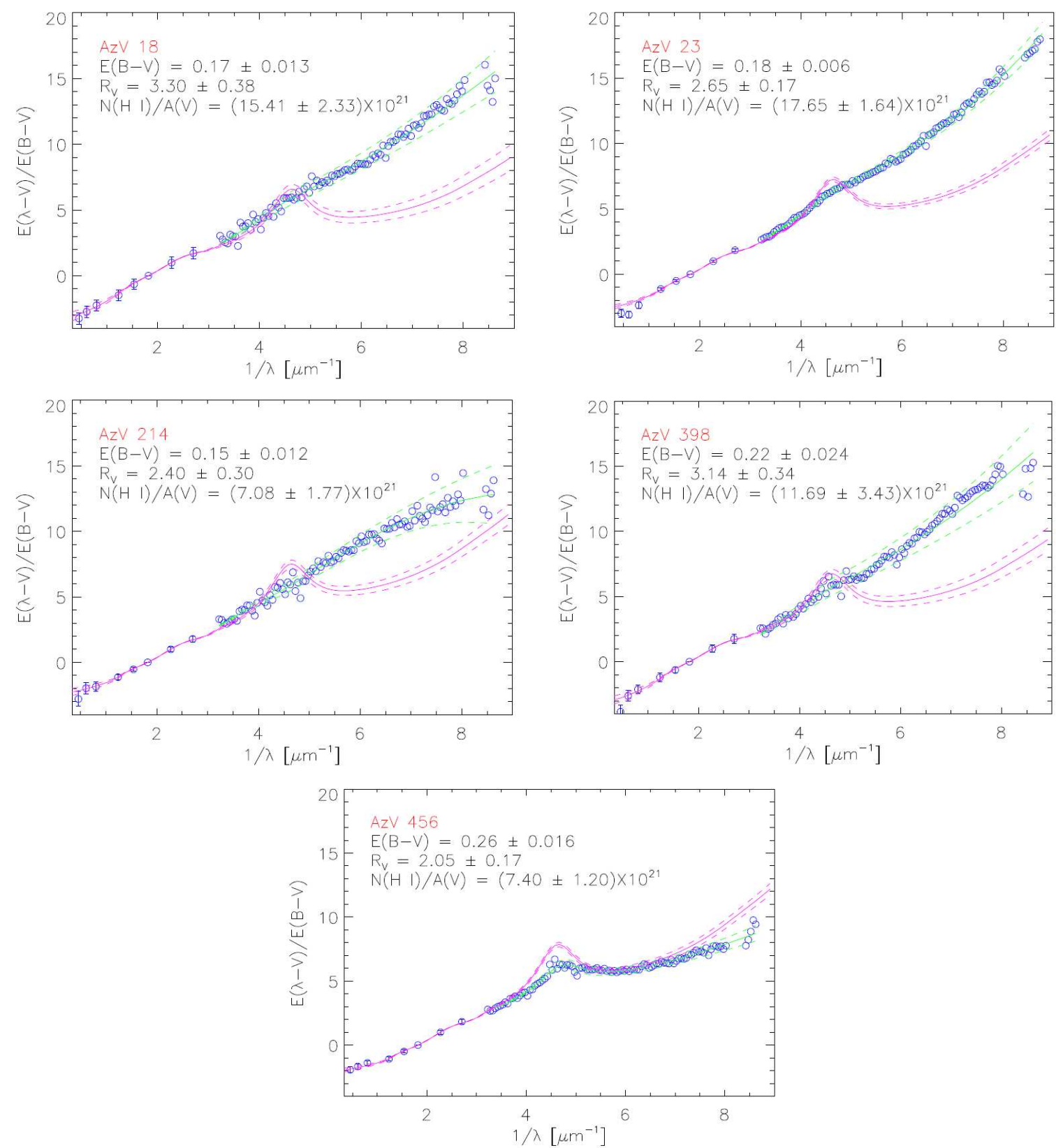

Figura 1.7: Curvas de extinção para a PNM retiradas de Gordon et al. (2003). A linha sólida verde é o melhor ajuste do modelo de Fitzpatrick e Massa (1990), e as linhas tracejadas verdes são as incertezas. A linha sólida rosa é a curva do modelo de Cardelli et al. (1989) para os valores de $R_{V}$ medidos, e as linhas tracejadas rosas são as incertezas. Os círculos azuis são os pontos observados. 


\subsubsection{A Ponte pan-Magelânica}

A evolução dinâmica do sistema PNM-GNM-VL parece ter gerado duas estruturas particularmente interessantes: a "Corrente Magelânica" (Magellanic Stream) e a "Ponte pan-Magelânica" (Magellanic Bridge).

A Corrente Magelânica é uma cauda de gás, e argumentam que esse gás pode ter sido arrancado da PNM devido a soma de dois efeitos - pressão de arraste e interação de maré das Nuvens de Magalhães com a Via Láctea. Já a Ponte pan-Magelânica parece ter sido produto dos mesmos efeitos só que entre a PNM e a GNM (Bekki, 2009), e além de ser formada de HI (Muller et al., 2004; Hindman et al., 1963), também possui nebulosidades em $\mathrm{H} \alpha$ (Sofue, 1994) e população estelar jovem (Irwin et al., 1985), que pode ter nascido já na Ponte e não ter sido arrancada das Nuvens.

Não se sabe com exatidão se as Nuvens de Magalhães se formaram como um sistema binário ou se elas se acoplaram dinamicamente há aproximadamente 4 Ganos atrás (Bekki e Chiba, 2005). A reconstrução da história cinemática das Nuvens sugere que a última aproximação entre elas ocorreu por volta de 0,2 Ganos atrás, na qual elas chegaram a uma distância entre $2-7 \mathrm{kpc}$, e a Ponte pan-Magelânica pode ter sido fruto dessa última "colisão" (Westerlund, 1991). Também acredita-se que a PNM pode ter sido esticada nas secções Nordeste e da Asa em direção à GNM depois desta última "colisão". Devido a isso, este trabalho se concentra no estudo dessas regiões.

Medidas fotoelétricas de Magalhães et al. (2009, 1990), Schmidt (1976, 1970) e Mathewson e Ford (1970b,a) sugeriram um campo magnético efetivamente alinhado com a Ponte pan-Magelânica. A emissão síncrotron polarizada, apesar de fraca, também confirma a existência de tal alinhamento (Haynes et al., 1990). Desse modo, o campo magnético pode ter tido um papel importante para a formação da Ponte. Esta hipótese é esperada, já que se observa material ionizado tanto nas Nuvens quanto na Ponte, e já foi provado que o campo magnético interestelar de galáxias pode ser forte o suficiente para influenciar a dinâmica do seu gás (Zweibel e Heiles, 1997). Assim, este trabalho tem também como objetivo estudar uma possível correlação entre o campo magnético e o gás para as regiões observadas. 
Capítulo 2

\section{Dados Observacionais}

Neste capítulo estão descritos a técnica observacional utilizada para determinar a polarização interestelar no óptico, o modo como foram realizadas as observações, os dados, e o modo como foi feita a redução destes.

\subsection{Polarimetria de Imagem Óptica}

A luz emitida por uma fonte qualquer pode ter natureza polarizada ou não. Na seção 1.2, já foram apresentados mecanismos físicos capazes de polarizar a luz, e também foram mencionados alguns objetos astrofísicos que emitem luz intrinsecamente polarizada. Porém, independente de como a luz se tornou linearmente polarizada, o objetivo dessa seção é discutir uma técnica capaz de medir essa polarização. No caso, os parâmetros de Stokes Q e U de um feixe de luz.

Como o propósito é medir a polarização, é necessária a utilização de um analisador que seja capaz de separar um feixe de luz em componentes de diferentes polarizações. Desse modo, pode-se utilizar um material que seja polarizador. Existem diversos tipos de materiais que se comportam como tal, e para tanto eles precisam ser dicroicos, refletores, espalhadores ou birrefringentes (Hecht, 2001). Um cristal que se enquadra em uma dessas categorias é a calcita, ou carbonato de cálcio $\left(\mathrm{CaCO}_{3}\right)$, que é um material birrefringente, ou seja, possui dois índices de refração, que separam a luz incidente nele em duas componentes - uma paralela e outra perpendicular ao seu plano de polarização, gerando duas imagens (ordinária e extraordinária). A vantagem de utilizar a calcita é que, devido ao fato dela duplicar a imagem da luz que a atravessa, a contribuição do fundo do céu em cada imagem deve ser aproximadamente a mesma, de modo à polarização do fundo do céu acabar se 
cancelando.

Para medir os parâmetros Q e U de um feixe, é necessário rotacionar a calcita de, pelo menos, quatro ângulos convenientes, como foi mencionado na seção 1.2.1. No entanto, do ponto de vista instrumental, é mais conveniente que a calcita seja mantida fixa, e que seja utilizado algum outro material capaz de mudar a direção de propagação da onda incidente e de maior flexibilidade para ser rotacionado. Um material que se enquadra nesses requisitos é um retardador. Como o interesse é medir luz linearmente polarizada, uma escolha adequada é a utilização de uma lâmina de meia-onda, um retardador que introduz uma fase de $180^{\circ}$ na luz incidente, ou seja, não destrói uma possível polarização linear. A utilização desse retardador faz com que a luz incidente fique rotacionada de um ângulo 2 $\theta$, como pode ser visto na figura 2.1, devido ao diferente índice de refração para as componentes ordinária e extraordinária da luz.

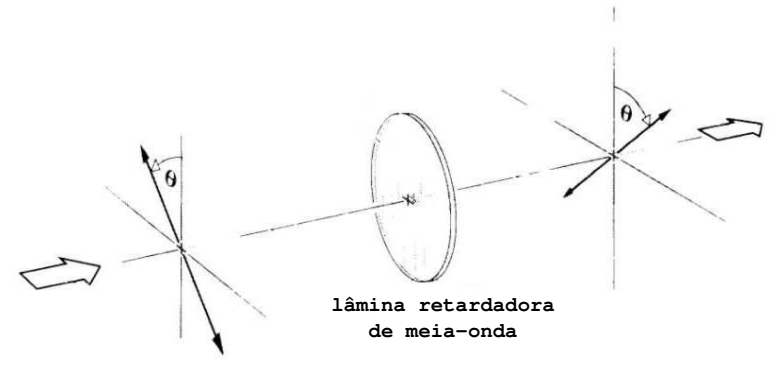

Figura 2.1: Lâmina retardadora de meia-onda rotaciona luz linearmente polarizada de um

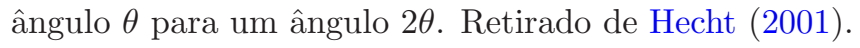

Assim sendo, um aparato instrumental capaz de medir o grau de polarização linear da luz é uma lâmina de meia-onda seguida de uma calcita. Como o interesse é obter a polarização no óptico, mais especificamente na banda V centrada em 540 nm, é necessário colocar um filtro V após a calcita, e para coletar a luz pode-se utilizar um CCD. A figura 2.2 é uma representação esquemática dessa configuração instrumental.

Utilizando tal aparato instrumental a intensidade da luz que chega ao detector é expressa por (Serkowski, 1974)

$$
I(\phi)=\frac{1}{2} I_{o}\{1 \pm[Q \cos (4 \phi)+U \operatorname{sen}(4 \phi)]\},
$$

onde $I_{o}$ é a intensidade total do feixe; e $\phi$ é o ângulo entre o eixo óptico da lâmina de meia-onda e o eixo óptico da calcita. A partir da equação 2.1, pode-se obter os parâmetros 


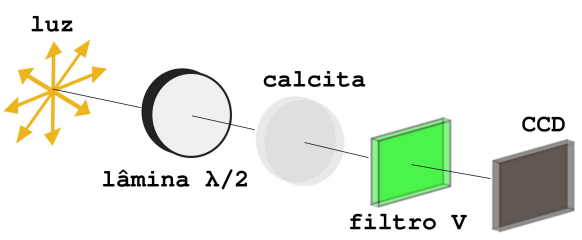

Figura 2.2: Configuração instrumental que pode ser utilizada para medir o grau de polarização linear de um feixe de luz.

de Stokes utilizando ângulos $\phi=n \times 22,5$ graus, sendo $n$ um número inteiro entre 0 e 15 .

\subsection{Observações}

Os dados foram obtidos pelo observador Antônio Mário Magalhães, nas noites entre 13 e 17 de novembro de 1992, no Cerro Tololo Inter-American Observatory (CTIO), empregando a técnica de polarimetria de imagem óptica no telescópio de 1,5 m. Foi utilizado um detector CCD Tek1K-1 de 1k x 1k, com escala de placa de 0,434 "/pixel, ruído de leitura de 8,0602 ADUs e ganho de 9,005 e-/ADU. Maiores detalhes sobre o aparato instrumental podem ser obtidos em Magalhães et al. (1996). Durante a missão foram utilizadas três seções do CCD, as quais estão listadas a seguir:

$$
\begin{aligned}
\text { Inteira } & \Rightarrow[1: 1064,1: 1024] \\
\text { Média } & \Rightarrow[1: 764,1: 300] \\
\text { Pequena } & \Rightarrow[1: 400,1: 300]
\end{aligned}
$$

Foram tomadas imagens de bias e flat-field para posterior eliminação dos ruídos instrumentais. A tabela 2.1 apresenta os parâmetros importantes para cada uma dessas imagens.

Tabela 2.1 - Parâmetros das imagens utilizadas para eliminação do ruído instrumental.

\begin{tabular}{ccccc}
\hline \hline Imagem & Seção & Noites & \# de imagens & TI (s) \\
\hline Bias & Inteira & 1 e 4 & 18 & Mínimo \\
Bias & Pequena & 2 e 4 & 29 & Mínimo \\
Bias & Média & 5 & 20 & Mínimo \\
Flat-field & Inteira & 1 & 10 & 20,0 \\
& & & & \\
\hline
\end{tabular}

\footnotetext{
7 TI é o tempo de integração.
} 
Foi observada uma estrela padrão não-polarizada para verificar se havia polarização instrumental, e estrelas padrões polarizadas para realizar a conversão dos ângulos de polarização para o sistema equatorial. A tabela 2.2 apresenta as informações relevantes e os parâmetros de observação para essas estrelas.

Tabela 2.2 - Informações relevantes e parâmetros de observação para as estrelas padrões.

\begin{tabular}{ccccccccc}
\hline \hline HD & AR $(\mathrm{J} 2000)$ & DEC $(\mathrm{J} 2000)$ & $m_{V}(\mathrm{mag})$ & Noite & Seção & $\mathrm{N}^{8}$ & TI $(\mathrm{s})^{7}$ & Padrão \\
\hline \multirow{2}{*}{9540} & $01: 33: 15,81$ & $-24: 10: 40,64$ & 7,0 & 1 & Pequena & $1 \times 16$ & 20,0 & Não-polarizada \\
283812 & $04: 44: 24,92$ & $+25: 31: 42,72$ & 9,6 & 1 & Pequena & $1 \times 16$ & 8,0 & Polarizada \\
23512 & $03: 46: 34,20$ & $+23: 37: 26,50$ & 8,2 & 2 & Pequena & $1 \times 16$ & 3,0 & Polarizada \\
23512 & $03: 46: 34,20$ & $+23: 37: 26,50$ & 8,2 & 3 & Pequena & $1 \times 4$ & 3,0 & Polarizada \\
23512 & $03: 46: 34,20$ & $+23: 37: 26,50$ & 8,2 & 4 & Média & $1 \times 4$ & 3,0 & Polarizada \\
23512 & $03: 46: 34,20$ & $+23: 37: 26,50$ & 8,2 & 5 & Média & $1 \times 4$ & 4,0 & Polarizada \\
298383 & $09: 22: 29,77$ & $-52: 28: 57,28$ & 9,8 & 5 & Média & $1 \times 4$ & 6,0 & Polarizada \\
& & & & & & & &
\end{tabular}

Foram observados 28 campos, com 8 × 8 minutos de arco, distribuídos nas secções Nordeste e da Asa da PNM e no início da Ponte pan-Magelânica. A tabela 2.3 apresenta as coordenadas deles e as noites em que foram observados, e a figura 2.3 apresenta suas localizações. Foi utilizada a seção inteira do CCD, tempo de integração de 300 segundos, e foi realizada uma exposição para quatro posições de lâmina $\left(\phi=0^{\circ}, 22,5^{\circ}, 45^{\circ}\right.$ e $\left.67,5^{\circ}\right)$.

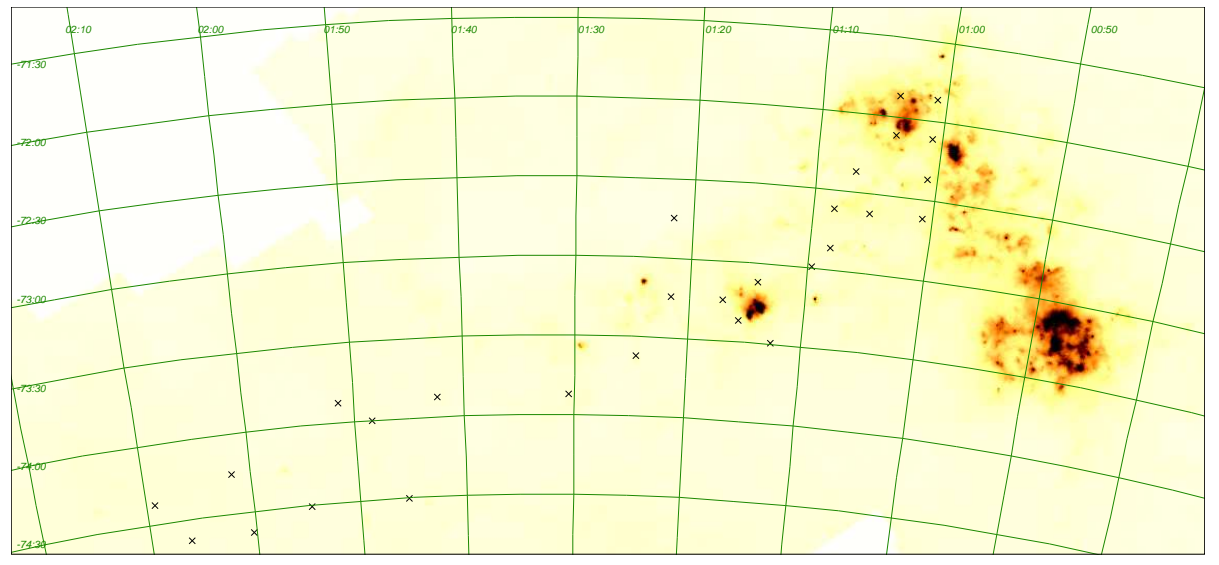

Figura 2.3: Campos observados sobrepostos a uma imagem do Spitzer/MIPS em $160 \mu \mathrm{m}$.

\footnotetext{
${ }^{8} \mathrm{~N}=$ \# de imagens $\mathrm{x}$ \# de posições da lâmina; 16 posições de lâmina significa todas as possíveis, ou seja, $\phi=22,5^{\circ} \times n$, com $n=(0,1, \ldots, 15) ; 4$ posições de lâmina significa $\phi=0^{\circ}, 22,5^{\circ}, 45^{\circ}$ e $67,5^{\circ}$.
} 
Tabela 2.3 - Coordenadas dos campos e noite em que foram observados.

\begin{tabular}{llll|llll}
\hline \hline Campo & AR $(\mathrm{J} 2000)$ & DEC $(\mathrm{J} 2000)$ & Noite & Campo & AR $(\mathrm{J} 2000)$ & DEC $(\mathrm{J} 2000)$ & Noite \\
\hline PNM01 & $01: 00: 50,18$ & $-71: 51: 55,21$ & 1 & PNM15 & $01: 17: 10,21$ & $-73: 14: 35,79$ & 2 \\
PNM02 & $01: 00: 49,78$ & $-72: 06: 56,21$ & 1 & PNM16 & $01: 21: 39,35$ & $-72: 44: 39,12$ & 5 \\
PNM03 & $01: 00: 49,37$ & $-72: 21: 55,20$ & 1 & PNM17 & $01: 21: 38,16$ & $-73: 14: 40,11$ & 3 \\
PNM04 & $01: 00: 48,95$ & $-72: 36: 56,20$ & 1 & PNM18 & $01: 24: 35,83$ & $-73: 37: 11,41$ & 4 \\
PNM05 & $01: 03: 48,90$ & $-71: 51: 56,95$ & 1 & PNM19 & $01: 30: 32,35$ & $-73: 52: 17,27$ & 3 \\
PNM06 & $01: 03: 48,48$ & $-72: 06: 57,95$ & 1 & PNM20 & $01: 42: 26,82$ & $-73: 52: 26,92$ & 3 \\
PNM07 & $01: 05: 16,92$ & $-72: 37: 27,85$ & 2 & PNM21 & $01: 45: 23,27$ & $-74: 30: 00,76$ & 3 \\
PNM08 & $01: 06: 46,73$ & $-72: 21: 59,77$ & 2 & PNM22 & $01: 48: 23,67$ & $-74: 00: 03,71$ & 3 \\
PNM09 & $01: 08: 15,60$ & $-72: 36: 59,72$ & 2 & PNM23 & $01: 51: 22,76$ & $-73: 52: 36,72$ & 4 \\
PNM10 & $01: 08: 15,11$ & $-72: 52: 00,71$ & 2 & PNM24 & $02: 01: 46,65$ & $-74: 15: 17,83$ & 3 \\
PNM11 & $01: 09: 44,18$ & $-72: 59: 31,67$ & 2 & PNM25 & $02: 00: 15,79$ & $-74: 37: 45,17$ & 5 \\
PNM12 & $01: 12: 41,72$ & $-73: 29: 33,66$ & 2 & PNM26 & $02: 06: 13,05$ & $-74: 37: 52,86$ & 3 \\
PNM13 & $01: 14: 11,86$ & $-73: 07: 03,69$ & 2 & PNM27 & $02: 09: 12,80$ & $-74: 22: 54,33$ & 3 \\
PNM14 & $01: 15: 40,61$ & $-73: 22: 04,73$ & 2 & PNM28 & $01: 54: 37,91$ & $-74: 30: 20,12$ & 3 \\
& & & & & & \\
\hline
\end{tabular}

\subsection{Redução dos Dados}

Os dados observacionais foram reduzidos pela autora deste trabalho, utilizando o software IRAF. Primeiramente as imagens foram tratadas para a eliminação dos ruídos instrumentais, em seguida os parâmetros polarimétricos foram obtidos, e por fim foi feita a calibração de magnitudes e os catálogos foram construídos. A seguir estão descritos os passos realizados.

\subsubsection{Tratamento das Imagens}

Inicialmente as imagens foram tratadas para correção de overscan, subtração do ruído de leitura e correção para variação de contagens pixel-a-pixel.

Se um CCD tivesse a mesma resposta para todos os pixeis, ao construir um gráfico das contagens em função da coluna (mantendo uma linha fixa), para uma imagem de flat-field, o padrão observado seria o de uma função degrau. Nele as colunas com maior contagem corresponde à área do CCD que pode ser considerada como confiável para redução e as colunas com menor contagem à faixa de overscan do CCD. Partindo desse princípio, foi 
utilizada uma imagem de flat-field e a tarefa implot, do pacote plot do IRAF, para determinar a área do CCD a ser utilizada para redução (trimsec) e a área de overscan (biassec), que é uma área do CCD que não fica exposta à luz e é utilizada como um patamar mínimo para que não existam contagens negativas. A tabela 2.4 apresenta a trimsec e biassec para cada seção do CCD utilizada.

Tabela 2.4 - Trimsec e biassec para cada seção do CCD utilizada.

\begin{tabular}{ccc}
\hline \hline Seção & Trimsec & Biassec \\
\hline Inteira & {$[2: 1024,2: 1023]$} & {$[1028: 1063,2: 1023]$} \\
Média & {$[5: 724,2: 300]$} & {$[741: 761,2: 300]$} \\
Pequena & {$[5: 360,2: 300]$} & {$[377: 397,2: 300]$} \\
& & \\
\hline
\end{tabular}

Após determinar essas seções, as imagens de bias foram corrigidas por overscan e trimsec, a partir da tarefa ccdproc do pacote noao/imred/ccdred. Essas imagens foram tomadas com o obturador do telescópio fechado e são importantes para a subtração do ruído de leitura do CCD. Foram feitas imagens considerando três seções diferentes: inteira, média e pequena, pois foram as seções utilizadas para a observação dos campos e estrelas padrões. Logo, as correções de overscan e trimsec dependeram da seção utilizada. As imagens de bias para cada caso foram combinadas empregando a tarefa zerocombine, também do pacote mencionado anteriormente. As imagens puderam ser combinadas para toda a missão, já que não foi observada nenhuma variação importante das contagens médias entre as noites.

O próximo passo foi corrigir as imagens de flat-field por overscan, trimsec e bias. O mesmo foi feito por meio da tarefa ccdproc. Então, as imagens de flat-field foram combinadas a partir da tarefa flatcombine, também do pacote anterior. Foi utilizada a seção inteira do CCD, e as imagens foram tomadas apenas na primeira noite, porque não era de se esperar grande variação no número médio de contagens e nem no padrão do flatfield. Essas imagens foram feitas iluminando uma tela branca com uma luz, assim espera-se que cada pixel esteja recebendo aproximadamente o mesmo número de fótons por unidade de tempo. Assim, pode-se utilizar essas imagens para corrigir a variação pixel-a-pixel, ou seja, normalizar a resposta de cada pixel dividindo as imagens dos objetos observados pelo 
flat-field.

Por fim, as imagens dos campos e das estrelas padrões foram corrigidas por overscan, trimsec, bias e flat-field, novamente por meio da tarefa ccdproc. As correções de trimsec, overscan e bias dependeram da seção do CCD que foi empregada. No caso da correção de flat-field, a tarefa ccdproc considerava automaticamente a seção certa a ser corrigida.

\subsubsection{Parâmetros Polarimétricos}

Após a eliminação dos ruídos instrumentais, as imagens estavam preparadas para a obtenção dos parâmetros polarimétricos, o que foi realizado por meio da tarefa quickpol do pacote pccdpack, desenvolvido pelo Grupo de Polarimetria do IAG/USP (Pereyra, 2000).

A tarefa quickpol executa consecutivamente todas as tarefas necessárias para que seja possível o cálculo dos parâmetros polarimétricos e pode ser utilizada nos modos para objeto individual ou campo denso. Os passos que a tarefa realiza são:

1. Daoedit/Daofind: No caso de objeto individual a tarefa daoedit é utilizada para que seja indicada a estrela a ser reduzida. Para campo denso a tarefa daofind é utilizada, a qual procura por todos os objetos do campo que possuem perfil estelar.

2. Ordem: Esta tarefa é executada apenas no modo de campo denso, e é necessária para ordenar em pares as estrelas encontradas pelo daofind. Vale lembrar que, por causa da calcita, a imagem de cada estrela se apresenta duplicada.

3. Daoedit/Daofind e ordem: No modo de campo denso existe a opção de reexecutar estas tarefas apenas para as estrelas brilhantes do campo, e então apenas essas estrelas são utilizadas para executar a tarefa seguinte.

4. Imalign: Alinha as imagens de todas as posições da lâmina com relação à primeira imagem. Este passo é necessário, pois o movimento do telescópio durante a observação pode causar algum deslocamento entre as imagens.

5. Coorshift: Obtém os deslocamentos entre as imagens e cria arquivos com as novas coordenadas para os objetos em cada imagem baseando-se nesses deslocamentos.

6. Phot: Realiza fotometria de abertura para todas as estrelas encontradas. Esta tarefa é executada separadamente para cada posição da lâmina. 
7. Txdump: Organiza os parâmetros necessários para polarimetria, obtidos pela fotometria, para cada imagem em arquivos.

8. Pccd: Calcula os parâmetros polarimétricos $\left(\mathrm{Q}, \mathrm{U}, \sigma_{Q, U, P}, \mathrm{P}\right.$ e $\left.\theta\right)$ para cada abertura utilizada pelo phot.

9. Macrol: Obtém a abertura que minimiza o erro polarimétrico.

10. Select: Neste caso utilizada para organizar os parâmetros importantes de cada estrela em um arquivo (coordenadas, informação polarimétrica e abertura utilizada).

Para executar a tarefa quickpol é necessário entrar com uma série de parâmetros, os quais estão listados na tabela 2.5. Além deles, também é imprescindível especificar se será empregado o modo de objeto individual ou campo denso, no caso de campo denso se serão utilizadas apenas estrelas brilhantes para executar a tarefa imalign; entrar com a imagem de referência; e a lista de imagens para todas as posições da lâmina.

Tabela 2.5 - Lista de parâmetros de entrada para a tarefa quickpol.

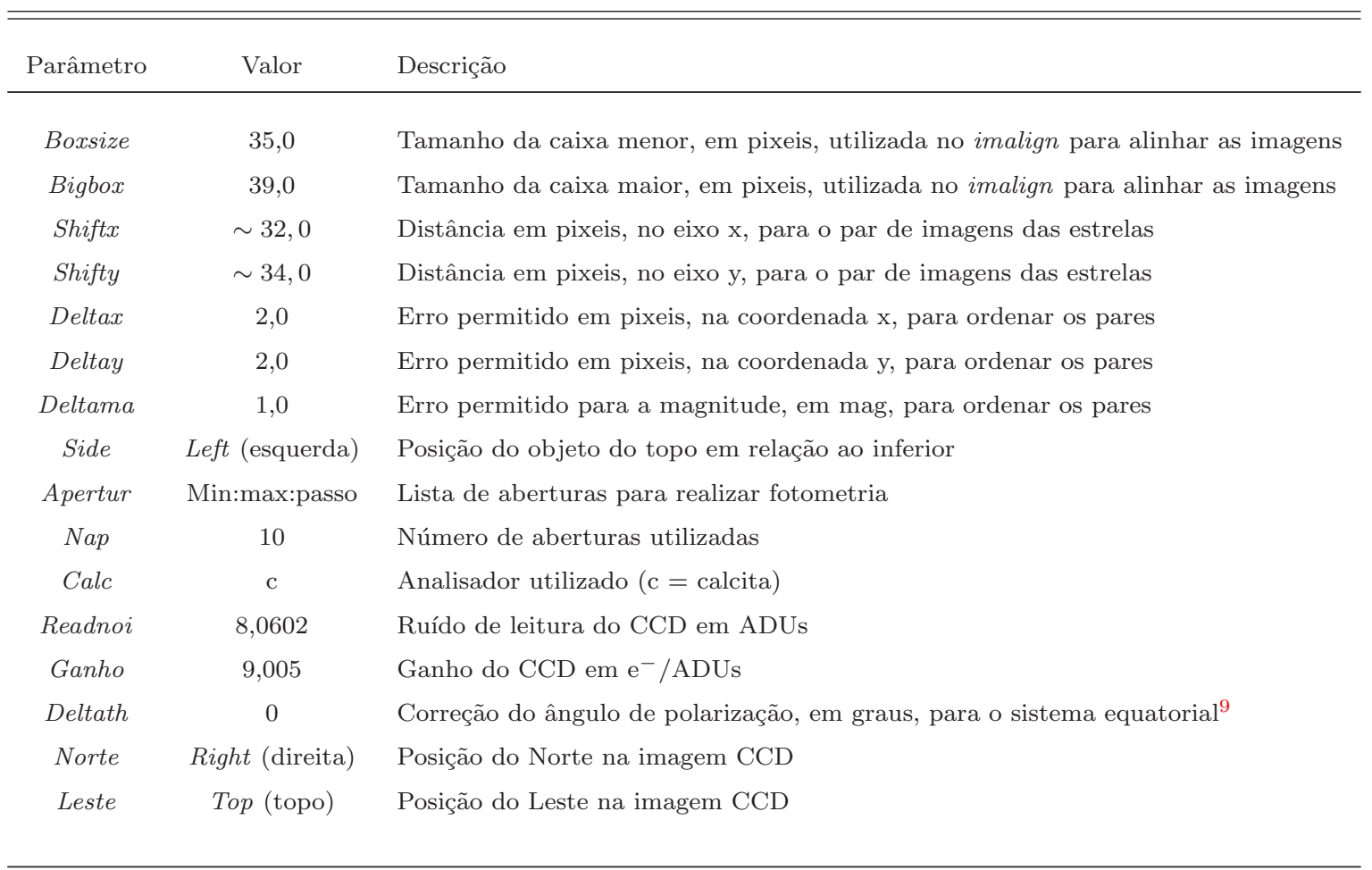

\footnotetext{
${ }^{9}$ A correção dos ângulos para o sistema equatorial foi realizada mais tarde.
} 
A boxsize e bigbox foram definidas como 35,0 e 39,0, respectivamente, porque notou-se ser um valor grande o suficiente para que não haja problema em encontrar as estrelas para realizar o alinhamento das imagens, porém pequeno o suficiente para que não seja feito um alinhamento pouco preciso.

Os parâmetros shiftx e shifty variaram um pouco de um campo para outro. Porém, eles foram aproximadamente os valores apresentados na tabela. Para estimar esses dois parâmetros para cada campo, utilizava-se a tarefa daoedit na imagem da primeira posição da lâmina (imagem de referência). Com essa tarefa era possível obter as coordenadas centrais dos pixeis para um par de imagens de uma estrela, e então os deslocamentos eram obtidos fazendo a diferença entre ambas. Para estimar esses valores em cada campo eram utilizadas três estrelas, que não fossem nem muito brilhantes e nem muito fracas, e então fazia-se uma média entre os deslocamentos obtidos para cada estrela.

Os erros permitidos de 2,0 pixeis para deltax e deltay e 1,0 mag para deltama foram escolhidos de forma a tentar minimizar a chance de um par ser mal definido, o que pode acontecer principalmente para uma região com muitas estrelas próximas.

Para estimar o parâmetro apertur era necessário conhecer a FWHM ${ }^{10}$ média dos perfis radiais das estrelas do campo. Para tanto também empregava-se a tarefa daoedit e as mesmas três estrelas citadas anteriormente. O valor mínimo (min) era definido partindo do princípio de que para estrelas fracas a abertura ótima em geral está em torno de 0,75×FWHM (Howell, 1992). Em seguida, escolhia-se como mínimo o maior valor inteiro igual a $0,75 \times \mathrm{FWHM}$, para conseguir uma boa abertura para os objetos fracos. O valor máximo era definido como $\max =\min +9$, e o passo utilizado era de 1 , de modo a aproveitar o número máximo possível de aberturas igual a 10.

A tarefa daoedit também mostrava o número de contagens do céu (sky) e a dispersão dela $\left(\sigma_{s k y}\right)$ em torno das estrelas. Isso era importante, porque os valores da $\sigma_{s k y}$ e também da FWHM eram solicitados pelas tarefas daofind e phot conforme o quickpol era executado.

Durante a execução da tarefa phot não era realizada recentragem dos objetos, pois notou-se que ela tendia a confundir os pares das estrelas em regiões com muitos objetos próximos. Estimava-se o raio interno do anel do céu como sendo $\sim 6 \times \mathrm{FWHM}$, visto que para objetos brilhantes, é necessário integrar até cerca de $5-6 \times$ FWHM do perfil radial

\footnotetext{
${ }^{10} \mathrm{FWHM}=$ full width at half maximun (largura a meia altura).
} 
para obter toda a luz emitida pela estrela (Howell, 1990). Escolhendo esse valor garante-se que o raio interno do céu não incluirá contagens do objeto, no caso de estrelas brilhantes. Já a largura do anel do céu era estimada como $\sim 2,5 \times \mathrm{FWHM}$, para que a contribuição de contagens devido a algum objeto que possa estar próximo não afete muito o valor do céu (Howell, 1990). Utilizava-se a moda das contagens dos pixeis, nesse anel, para definir o valor do céu. Não eram fixados valores mínimos e máximos de contagens que uma estrela pode ter para realizar a fotometria, pois não era desejado cortar nenhum objeto nesta parte da redução.

\subsubsection{Estrelas Padrões}

Para obter os parâmetros polarimétricos das estrelas padrões foi empregado o modo de objeto individual do quickpol. Anteriormente foi explicado como é realizada a execução dessa tarefa e como são definidos os parâmetros de que ela necessita.

Primeiramente foi reduzida a estrela padrão não-polarizada HD 9540. Como foi obtida polarização compatível dentro de $1 \sigma$ com valor publicado (Heiles, 2000), a correção de polarização instrumental não se fez necessária. Em seguida, foi realizada a redução das estrelas padrões polarizadas, a fim de obter a correção $\Delta \theta$ para os ângulos de polarização ao sistema equatorial. A tabela 2.6 apresenta os resultados obtidos para essas estrelas e valores publicados.

Tabela 2.6 - Parâmetros polarimétricos obtidos $\left(P_{o b s}\right.$ e $\left.\theta_{o b s}\right)$ e valores publicados $\left(P_{p u b}\right.$ e $\left.\theta_{p u b}\right)$ para as estrelas padrões, e correção $\Delta \theta$ do ângulo de polarização ao sistema equatorial.

\begin{tabular}{cc|cc|cc|c}
\hline \hline HD & Noite & $P_{\text {obs }}(\%)$ & $\theta_{\text {obs }}$ (graus) & $P_{\text {pub }}(\%)$ & $\theta_{\text {pub }}$ (graus) & $\Delta \theta$ (graus) \\
\hline \multirow{2}{*}{9540} & 1 & $(0,035 \pm 0,027)$ & 145,5 & $(0,030 \pm 0,019)$ & 37,4 & - \\
283812 & 1 & $(6,29 \pm 0,11)$ & 120,6 & $(6,091 \pm 0,023)$ & 31,8 & $(-88,80 \pm 0,52)$ \\
23512 & 2 & $(2,18 \pm 0,12)$ & 121,3 & $(2,198 \pm 0,061)$ & 30,1 & $(-91,2 \pm 1,8)$ \\
23512 & 3 & $(2,253 \pm 0,037)$ & 119,1 & $(2,198 \pm 0,061)$ & 30,1 & $(-89,00 \pm 0,93)$ \\
23512 & 4 & $(2,271 \pm 0,050)$ & 118,3 & $(2,198 \pm 0,061)$ & 30,1 & $(-88,2 \pm 1,0)$ \\
23512 & 5 & $(2,377 \pm 0,029)$ & 117,2 & $(2,198 \pm 0,061)$ & 30,1 & $(-87,10 \pm 0,87)$ \\
298383 & 5 & $(5,012 \pm 0,065)$ & 54,7 & $(5,2330 \pm 0,0090)$ & 148,61 & $(-86,09 \pm 0,37)$ \\
& & & & & &
\end{tabular}

É importante comentar que foi escolhido como erro para Q, U e P o valor máximo entre 
o erro calculado e o erro teórico.

Utilizou-se como referência o valor publicado por HPOL para a estrela padrão polarizada HD 283812, o publicado por Tapia (1988) para a HD 298383 e os valores publicados por Bastien et al. (1988) e Hsu e Breger (1982) para a HD 23512. No caso desta última estrela, inicialmente obtiveram-se as duas estimativas para os parâmetros Q e U a partir dos diferentes P e $\theta$ publicados. Então, fez-se uma média ponderada pelas incertezas para Q e U. Por fim, foram calculados $\langle P\rangle$ e $\langle\theta\rangle$ a partir dos valores de $\langle Q\rangle$ e $\langle U\rangle$. O erro para $\langle P\rangle$ foi definido como o desvio padrão da média para os valores de $\langle Q\rangle$ e $\langle U\rangle$, pois o que é obtido pela média ponderada pelas incertezas costuma levar a um erro subestimado. Vale lembrar que o erro para $\theta$ pode ser calculado por $\sigma_{\theta}=28^{\circ}, 65 \times \sigma_{P} / P$ (Serkowski, 1974).

Nota-se que todas as estrelas possuem polarizações compatíveis com o valor publicado dentro de $3 \sigma$, com exceção da HD 298383. No caso dessa estrela, ela é compatível apenas dentro de $\sim 3,4 \sigma$. Isso acontece provavelmente devido à incerteza para o valor publicado ser muito baixa. Mas, ainda assim, a diferença está dentro de um limite aceitável. Portanto, têm-se uma boa indicação de que o instrumento mediu as polarizações de forma acurada.

Todavia, o objetivo principal da observação das padrões polarizadas é determinar a correção $\Delta \theta$ para os ângulos de polarização ao sistema equatorial. Isso é feito calculandose $\Delta \theta=\theta_{p u b}-\theta_{o b s}$ ou $\Delta \theta=\theta_{p u b}-\theta_{o b s}-180^{\circ}$ para cada uma das padrões polarizadas observadas. Essa segunda opção é considerada no caso da obtenção de um valor de $\Delta \theta>0$, o que aconteceu para a estrela HD 298383. As incertezas foram estimadas por propagação de incertezas, quer dizer, $\sigma_{\Delta \theta}=\sqrt{\sigma_{\theta_{p u b}}^{2}+\sigma_{\theta_{o b s}}^{2}}$.

Por fim, foi feita uma média ponderada pelas incertezas para os valores obtidos para $\Delta \theta$, e a incerteza foi estimada como sendo o desvio padrão da média, novamente para evitar a obtenção de um erro subestimado. A correção para os ângulos de polarização ao sistema equatorial obtida foi de $\Delta \theta=(-87,33 \pm 0,72)$ graus.

\subsubsection{Campos na PNM}

Os parâmetros polarimétricos das estrelas presentes nos 28 campos da PNM foram estimados com o modo de campo denso do quickpol. Como foi mencionado anteriormente, a tarefa daofind busca por todos os objetos do campo que possuem perfil estelar. Foi definido que os objetos deveriam ter uma contagem mínima de $s k y+6 \times \sigma_{s k y}$ para que ele 
fosse considerado, e uma contagem máxima de 25.000 ADUs, que é um valor um pouco abaixo da saturação do CCD. Para saber o valor da saturação, foram analisados os perfis radiais de estrelas brilhantes em alguns campos.

Foi escolhido executar o alinhamento das imagens de cada posição da lâmina utilizando apenas as estrelas brilhantes do campo. Para tanto, definiu-se que uma estrela brilhante deve ter uma contagem de no mínimo 5.000 ADUs e no máximo 25.000 ADUs (ou seja, abaixo do nível de saturação). Também foi definido que um par de imagens de uma estrela deve estar há pelo menos 30 pixeis de distância da borda do CCD para evitar que alguma das imagens não esteja totalmente incluída no campo.

Assim, utilizando a saída das tarefas ordem e macrol executava-se a tarefa select, porém desta vez escolhendo incluir apenas as estrelas que possuem $P / \sigma_{P}>3$, e adicionando a correção $\Delta \theta$ para que os ângulos de polarização das estrelas ficassem no sistema equatorial.

Por fim, era realizado um primeiro teste dos resultados. Através da saída do select e um script escrito em IDL, procurava-se por todas as estrelas que possuíam $P \geq 5 \%$ ou coordenadas muito próximas, sendo definido como coordenadas muito próximas

$$
\left\{\begin{array}{l}
\Delta x=\left|x_{\star 1}-x_{\star 2}\right| \leq 5 \text { pixeis } \\
\Delta y=\left|y_{\star 1}-y_{\star 2}\right| \leq 5 \text { pixeis }
\end{array}\right.
$$

onde $\left(x_{\star 1}, y_{\star 1}\right)$ são as coordenadas da estrela $1 ;\left(x_{\star 2}, y_{\star 2}\right)$ as coordenadas da estrela 2 ; e as duas condições deveriam ser satisfeitas simultaneamente.

Este script gerava uma saída contendo as coordenadas, polarização, erro da polarização, abertura e número da estrela para as estrelas que satisfaziam a algum dos dois casos acima (P alta ou coordenadas próximas). Em seguida, utilizava-se a tarefa checkcen do pccdpack para conferir se essas estrelas apresentavam algum problema. Sendo consideradas como estrelas problemáticas:

- Objetos que possuíam coordenadas mal centradas. O que acontecia principalmente em regiões com muitas estrelas. Assim, a polarização obtida era equivocada, pois não considerava-se toda a luz emitida por um ou ambos pares, ou então somava-se a algum dos pares a luz emitida por algum outro objeto muito próximo.

- Estrelas brilhantes para as quais a abertura com menor erro era muito pequena. Isso pode ser impróprio, pois não era considerada toda a luz emitida pela estrela. Objetos 
desse caso em geral eram contados mais de uma vez, então esse problema podia ser identificado para casos em que as coordenadas eram muito próximas.

- Estrelas que simplesmente "não existiam". Na verdade notava-se que apesar delas terem sido contadas os seus sinais eram muito fracos. Esse era o caso que em geral levava a polarizações muito altas.

Por fim, executava-se novamente o select utilizando $P / \sigma_{P}>3$, corrigindo os ângulos de polarização por $\Delta \theta$ e retirando as estrelas que satisfaziam alguma das condições anteriores e realmente apresentavam problemas. Com isso, os parâmetros polarimétricos das estrelas de cada campo ficavam determinados oferecendo certa segurança de que os resultados eram acurados.

\subsubsection{Catálogos Polarimétricos}

Tendo em mãos os parâmetros polarimétricos, o próximo passo foi a obtenção dos catálogos, os quais contêm posições, polarização e seu erro, ângulo de polarização e magnitude na banda $\mathrm{V}$ e seu erro.

\subsubsection{Calibração das Posições}

Primeiramente foi necessário realizar a calibração das posições das estrelas, para tanto foi empregada a tarefa refer do pccdpack. Lembrando que devido à calcita as imagens de cada estrela se apresentam duplicadas, se torna necessário fazer a conversão das posições das estrelas na imagem CCD em relação a alguma imagem de referência. No caso foram utilizadas imagens do Digital Sky Server (DSS), que possuíam o mesmo centro das imagens CCD e com 8 × 8 minutos de arco.

A tabela 2.7 apresenta a lista de parâmetros fixos que eram necessários para executar o refer. Além deles, também era preciso dar como entrada a saída da tarefa select, a qual apresenta os parâmetros polarimétricos e posições do par superior das estrelas de cada campo; informar as coordenadas em pixeis de uma estrela de referência nas imagens CCD e $D S S$, para tanto sempre tentava-se utilizar uma estrela próxima do centro, e para obter as coordenadas desta em cada imagem empregava-se a tarefa daoedit; e informar o arquivo fits da imagem de referência. 
Tabela 2.7 - Lista dos parâmetros fixos dados como entrada para a tarefa refer.

\begin{tabular}{ccl}
\hline \hline Parâmetro & Valor & Descrição \\
\hline Epimg & 0,468 & Escala de placa da imagem DSS em "/pixel \\
Epccd & 0,434 & Escala de placa da imagem CCD em "/pixel \\
Ximagem & 1024 & Tamanho da imagem DSS na direção x, em pixeis \\
Yimagem & 1024 & Tamanho da imagem DSS na direção y, em pixeis \\
Xside & 1023 & Tamanho da imagem CCD na direção x, em pixeis \\
Yside & 1022 & Tamanho da imagem CCD na direção y, em pixeis \\
Norte & Right (direita) & Posição do Norte na imagem CCD \\
Leste & Top (topo) & Posição do Leste na imagem CCD \\
Incli & 0 & Angulo em graus da imagem CCD com respeito ao sistema equatorial \\
Recen & Yes (sim) & Parâmetro para ativar ou desativar a recentragem das posições das estrelas \\
Cbox & 15 & Tamanho em pixeis da caixa utilizada para recentragem \\
\end{tabular}

Foi realizada a recentragem das posições das estrelas na imagem de referência, pois notou-se que esse procedimento melhorava consideravelmente a precisão de suas posições. Porém, fazia com que algumas estrelas ficassem com coordenadas iguais, o que acontecia no caso de uma estrela brilhante e uma mais fraca muito próximas, ou alguma região com muitas estrelas próximas.

Essa tarefa partia da coordenada inicial obtida pela transformação que leva as coordenadas dos objetos na imagem CCD para o mesmo objeto na imagem $D S S$, para realizar a recentragem. Então, ela procurava pelo pixel que possuía maior contagem dentro de uma caixa (cbox), que era centrada nessa coordenada inicial. Por fim, a nova coordenada era a do pixel com maior contagem, supondo que ela seja equivalente à posição do pico do perfil radial da estrela. Verificou-se que a utilização de $c b o x=15$ era o melhor valor para recentrar as coordenadas evitando ao máximo que a nova posição coincidisse com alguma outra estrela.

Em seguida, empregava-se a tarefa correfer do pccdpack para tentar corrigir as coordenadas que apresentavam-se duplicadas, para os campos que possuíam estrelas com esse problema. Eram dados como entrada as saídas das tarefas select e refer e permitia-se uma distância máxima de 0,5 pixeis entre as coordenadas de cada estrela. Ou seja, essa tarefa tentava corrigir coordenadas com uma diferença menor ou igual a 0,5 pixeis. Verificou-se que a utilização de $c b o x=10$ era o melhor valor para corrigir o maior número possível de 
coordenadas iguais sem aumentar muito o erro nas posições. Contudo, ainda assim restaram estrelas com problema, e diminuir ainda mais o tamanho da caixa levava a posições pouco precisas.

\subsubsection{Obtenção das Magnitudes e Catálogos}

O próximo passo foi a obtenção e calibração das magnitudes. Para tanto, primeiramente era obtido o catálogo polarimétrico contendo as magnitudes instrumentais, o que era feito por meio das tarefas magnit e fintab do pccdpack.

A tarefa magnit calcula as magnitudes e os seus erros utilizando como entrada as saídas das tarefas phot e select. A segunda serve para obter as coordenadas de cada estrela e a abertura empregada para calcular os parâmetros polarimétricos, e a primeira, para verificar as contagens da estrela e do céu para dada abertura. Era utilizada a saída do phot para a primeira posição da lâmina. Desse modo, a magnitude $\left(m_{V}\right)$ e erro $\left(\sigma_{m_{V}}\right)$ para cada estrela podiam ser obtidas através das equações 2.4 e 2.5. A saída dessa tarefa guarda as magnitudes e seus erros e os parâmetros polarimétricos de cada estrela (contidos na saída do select).

$$
\begin{aligned}
m_{V} & =\operatorname{zmag}-2,5 \times \log _{10}(S-A \times m s k y)+2,5 \times \log _{10}(T I), \\
\sigma_{m_{V}} & =1,0857 \times \frac{\sqrt{\frac{(S-A \times m s k y)}{\text { ganho }}+A \times \sigma^{2}+\frac{A^{2} \times \sigma^{2}}{n s k y}}}{(S-A \times m s k y)},
\end{aligned}
$$

onde zmag é o ponto zero da escala de magnitudes; $S$ é a soma das contagens das imagens ordinária e extraordinária; $A$ é a área da abertura empregada; msky é a magnitude do céu em ADU/pixel, calculada através da média para as imagens ordinária e extraordinária; TI é o tempo de integração utilizado; ganho é o ganho do CCD em e ${ }^{-} / \mathrm{ADU} ; \sigma$ é o erro da magnitude do céu, obtido por propagação de incertezas considerando os erros para imagem ordinária e extraordinária; nsky é a média do número de pixeis usados para o cálculo do céu para as imagens ordinária e extraordinária. A equação 2.4 provém da definição de magnitude, e a equação 2.5 da propagação de incertezas considerando os erros envolvidos nas variáveis da equação $2.4^{11}$.

A tarefa fintab servia para a construção do catálogo, a partir das saídas do correfer (ou refer) e magnit e da imagem de referência do DSS. A saída do correfer (ou refer) e o

\footnotetext{
${ }^{11}$ Essa explicação está contida no help da tarefa magnit.
} 
cabeçalho da imagem $D S S$ eram utilizados para obter a AR e a DEC de cada estrela, já a saída do magnit para capturar as magnitudes e seus erros e os parâmetros polarimétricos. Assim, o catálogo gerado contém: AR, DEC, P, $\sigma_{P}, \theta, m_{V}$ e $\sigma_{m_{V}}$.

Em seguida, para calibrar as magnitudes das estrelas, utilizou-se catálogos que continham magnitudes na banda $\mathrm{V}$ para estrelas pertencentes aos campos considerados. A tabela 2.8 apresenta a referência dos catálogos e o número de estrelas utilizadas para a realização dessa calibração para cada campo.

Tabela 2.8 - Número de estrelas utilizadas e referências dos catálogos considerados para calibrar as magnitudes na banda $\mathrm{V}$ para cada campo.

\begin{tabular}{ccl}
\hline \hline PNM & \# estrelas & Referência \\
\hline $01-19$ & 4 & Massey (2002) \\
$20-21$ & 1 & Ardeberg e Maurice (1977), Isserstedt (1978) e Lasker et al. (2007) \\
22 & 1 & Isserstedt (1978) e Ardeberg (1980) \\
23 & 2 & Lasker et al. (2007) e Sanduleak (1989) \\
24 & 1 & Lasker et al. (2007) e Perie et al. (1991) \\
25 & 1 & Lasker et al. (2007) \\
$26-27$ & 3 & Demers e Irwin (1991) \\
28 & 2 & Lasker et al. (2007) \\
& & \\
\hline
\end{tabular}

Desse modo, a correção para magnitude e seu erro eram obtidos através de

$$
\begin{aligned}
\Delta m_{V} & =m_{V_{p u b}}-m_{V_{i n s}}, \\
\sigma_{\Delta m_{V}} & =\sqrt{\sigma_{m_{V_{p u b}}}^{2}+\sigma_{m_{V_{i n s}}}^{2}},
\end{aligned}
$$

onde $m_{V_{p u b}}$ e $\sigma_{m_{V_{p u b}}}$ são a magnitude na banda $\mathrm{V}$ e seu erro publicados; $m_{V_{\text {ins }}}$ e $\sigma_{m_{V_{i n s}}}$ são a magnitude na banda $\mathrm{V}$ e seu erro instrumentais. $\mathrm{O}$ valor de $\Delta m_{V}$ final, para cada campo, foi estimado como a média entre os $\Delta m_{V}$ de cada estrela de um campo, ou a média entre as diferentes estimativas de $\Delta m_{V}$ para uma única estrela. O erro foi definido como sendo o desvio padrão. Não foi utilizado o desvio padrão da média, pois nesse caso o valor do erro estaria sendo muito subestimado, já que o uso de um número pequeno de estrelas para estimativa dessa correção pode levar a uma calibração pouco precisa.

O emprego de correções diferentes para cada campo, ao invés de uma global, foi importante, pois notou-se que essas calibrações variaram um pouco de um campo para outro, 
como pode ser visto no gráfico da figura 2.4. Essa diferença ocorre provavelmente devido a mudanças nas condições fotométricas do céu durante a noite, entre as noites, ou para diferentes regiões do céu.

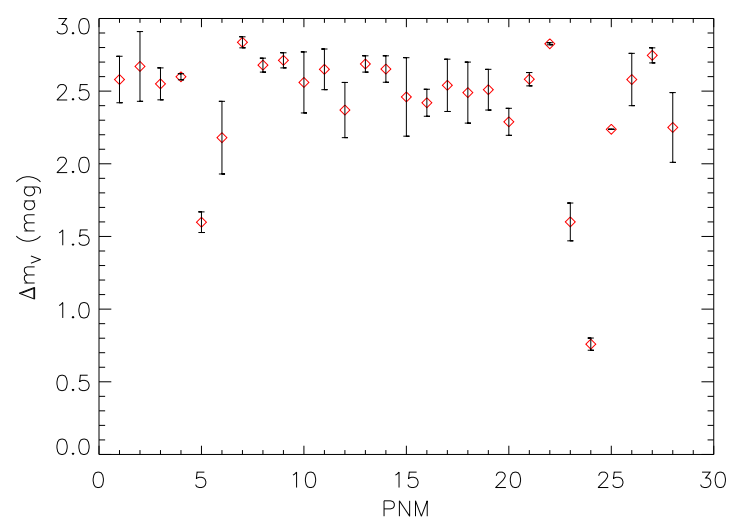

Figura 2.4: Correções $\Delta m_{V}$ para magnitude para cada um dos campos.

Por fim, para obter um catálogo com magnitudes na banda $\mathrm{V}$ no sistema padrão, executava-se novamente as tarefas magnit e fintab, porém dessa vez entrando com a correção para a magnitude, $\Delta m_{V}$ e sua incerteza, ao executar o magnit. O erro médio final para as magnitudes considerando todas as estrelas dos 28 campos foi de $\sigma_{m_{V}}=0,13$ mag.

\subsubsection{Estimativa dos Erros para Ascensão Reta e Declinação}

A estimativa dos erros para AR e DEC foi realizada utilizando como referência as coordenadas das estrelas consideradas para calibração das magnitudes, a partir da seguinte expressão

$$
\begin{aligned}
\sigma_{\mathrm{AR}} & =\frac{1}{N} \sum_{i=1}^{N}\left|\mathrm{AR}_{p u b, i}-\mathrm{AR}_{r e d, i}\right|, \\
\sigma_{\mathrm{DEC}} & =\frac{1}{N} \sum_{i=1}^{N}\left|\mathrm{DEC}_{p u b, i}-\mathrm{DEC}_{r e d, i}\right|,
\end{aligned}
$$

onde $\mathrm{AR}_{p u b, i}$ e $\mathrm{DEC}_{p u b, i}$ são as coordenadas publicadas da estrela $i$; $\mathrm{AR}_{r e d, i}$ e $\mathrm{DEC}_{r e d, i}$ são as coordenadas obtidas pela redução para estrela $i$; N é o número de estrelas consideradas ou as diferentes estimativas de AR e DEC publicadas para uma mesma estrela.

A partir do gráfico da figura 2.5, pode-se perceber a variação dos erros nas coordenadas para cada campo. A média do erro obtida para ascensão reta foi de $\sigma_{\mathrm{AR}}=0,25 \mathrm{~s}$, e para declinação de $\sigma_{\mathrm{DEC}}=1,0 "$. 

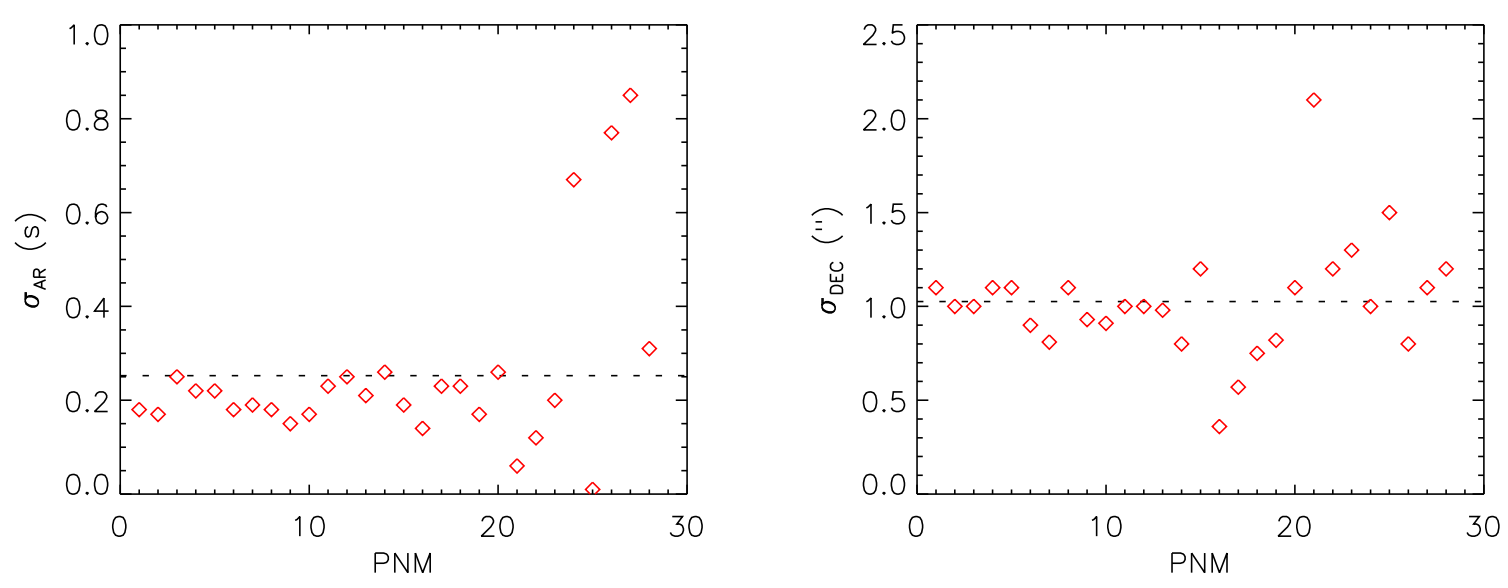

Figura 2.5: Erros nas coordenadas para cada campo. Esquerda - AR, direita - DEC.

O catálogo final, considerando os 28 campos, contém 7.207 estrelas com $P / \sigma_{P}>3$. Apesar de todo o esforço para tentar determinar as posições com precisão, $12 \%$ das estrelas do catálogo apresentam coordenadas repetidas. Verificou-se que esse problema ocorreu para casos em que existia uma estrela brilhante e uma fraca muito próximas, ou regiões onde a densidade de estrelas era alta. Esse catálogo está disponibilizado no endereço http://www . astro.iag.usp.br/ polarimetria/survey/. 
Capítulo 3

\section{Estrutura do Campo Magnético na PNM}

Neste capítulo está descrito como foi determinada e eliminada a polarização de foreground, é feita uma análise do catálogo polarimétrico, está apresentado como foram construídos os mapas dos padrões principais de polarização e como foi determinada a intensidade do campo magnético.

\subsection{Polarização de Foreground}

Antes de iniciar a análise do catálogo polarimétrico e da estrutura do campo magnético na PNM, se fez necessário realizar a subtração da polarização de foreground para as estrelas dos catálogos gerados. Essa correção é requerida, pois a luz das estrelas, ao sair da PNM, ainda percorre uma camada de poeira presente na nossa Galáxia. Assim, para obter a polarização intrínseca das estrelas devida apenas à poeira presente na PNM, deve-se subtrair a parte da polarização gerada na VL.

Schmidt (1976) utilizou medidas fotoelétricas de estrelas de foreground para estimar essa polarização. Ele dividiu a PNM em cinco regiões - quatro pertencentes ao corpo da PNM e uma no início da Ponte pan-Magelânica, e então realizou estimativas diferentes para cada uma dessas regiões. A figura 3.1 apresenta essa divisão.

Mais tarde, Rodrigues et al. (1997) avaliaram novamente essa polarização, também através de medidas fotoelétricas para estrelas de foreground e considerando a mesma divisão feita por Schmidt (1976), e obtiveram uma estimativa mais precisa dela.

Antes de subtrair a polarização de foreground para as estrelas dos catálogos, o primeiro passo foi determinar a qual região cada uma pertence. Para tanto, foi escrito um script em IDL que, a partir das coordenadas de cada estrela, verifica a qual das 5 regiões ela 


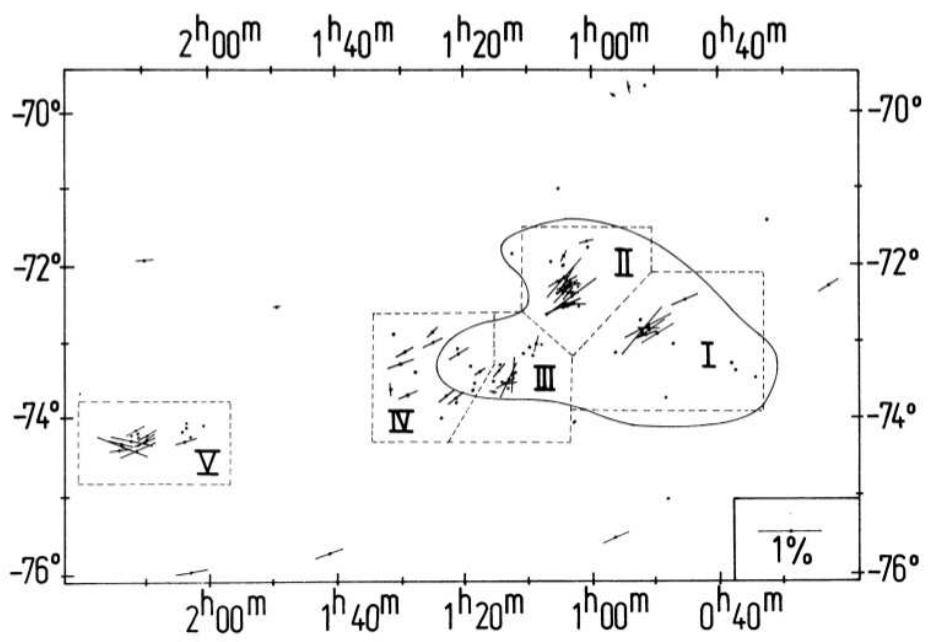

Figura 3.1: Divisão da PNM feita por Schmidt (1976) para realizar estimativas locais para polarização de foreground. Os vetores representam a polarização e seu ângulo para cada estrela considerada. Coordenadas precessionadas para 1975.

pertence. Primeiramente a coordenada era precessionada para 1975, e em seguida baseado nos limites de AR e DEC de cada região, era determinado em qual delas a estrela se encaixava. A tabela 3.1 apresenta a qual região a coordenada central de cada campo pertence. É necessário citar que para campos próximos de fronteiras aconteceu de existirem estrelas que pertenciam a diferentes regiões. Verificou-se que as estrelas de 5 campos não se encaixavam em nenhuma das regiões, os quais estavam entre as regiões IV e V, a partir de agora denominados como região IV-V. Assim, foi necessário pensar numa maneira alternativa para que fosse possível a determinação do foreground para ela.

Tabela 3.1 - Regiões de Schmidt (1976) às quais cada campo pertence.

\begin{tabular}{cc}
\hline \hline Região & PNM \\
\hline I & Nenhum \\
II & $01-10$ \\
III & $11-14$ \\
IV & $15-19$ \\
IV-V & $20-23$ e 28 \\
V & $24-27$ \\
& \\
\hline
\end{tabular}




\subsubsection{Determinação da Polarização de Foreground}

Para que fosse possível a subtração do foreground para as estrelas pertencentes à região IV-V, foi necessário pensar numa forma de estimar a polarização de foreground para essa região. Percebemos que isso poderia ser feito a partir dos nossos dados, o que acabou sendo útil para uma nova estimativa dessa polarização para as outras regiões também.

Levando em conta que os objetos presentes em cada um dos campos inclui não apenas estrelas pertencentes à PNM, mas também estrelas de foreground, quer dizer, localizadas entre a PNM e nós, observa-se que uma forma de estimar essa polarização a partir dos nossos dados é filtrar quais são as estrelas de foreground, e depois valendo-se delas para estimá-la.

Para realizar essa seleção partimos da definição do módulo de distância

$$
m_{V}-M_{V}=5 \log d-5+A_{V}
$$

onde $m_{V}$ é a magnitude aparente na banda $\mathrm{V} ; M_{V}$ é a magnitude absoluta na banda $\mathrm{V} ; d$ é a distância em pc; e $A_{V}$ é o avermelhamento na banda $\mathrm{V}$.

A distância até a PNM é conhecida, $d=(63 \pm 1)$ kpc (Cioni et al., 2000). Para estimar o avermelhamento médio, podemos utilizar o excesso de cor médio de foreground, $E(B-V) \simeq 0,05 \mathrm{mag}$ (Bessell, 1991), juntamente com a lei de extinção média para VL, $A_{V} \simeq 3,2 E(B-V)$ (Seaton, 1979), o que resulta em um avermelhamento médio de foreground de $A_{V} \simeq 0,2$ mag. Desse modo, o módulo de distância para a PNM é de $m_{V}-M_{V} \simeq 19,2$.

Ao atentarmos para o diagrama HR (ver figura 3.2), nota-se que apenas as estrelas gigantes e supergigantes mais brilhantes possuem $M_{V}<-5$ mag. Para que estrelas desse tipo possam pertencer à PNM, elas devem apresentar $m_{V} \lesssim 14,2$ mag, considerando o módulo de distância obtido anteriormente. O catálogo de Massey (2002) teve como objetivo calibrar com precisão as magnitudes de estrelas brilhantes na PNM, para complementar outros catálogos concentrados em estrelas mais fracas. Ele possui 84.995 estrelas com $m_{V} \lesssim 18 \mathrm{mag}$, das quais apenas 3,5\% apresentam $m_{V}<14,2 \mathrm{mag}$. Assim, a fração de estrelas super brilhantes deve ser bem pequena quando comparada ao resto.

Levando em consideração o que foi discutido até aqui e o fato de que todas as estrelas da VL utilizadas por Schmidt (1976) e Rodrigues et al. (1997) para determinar a polarização 
de foreground possuem $m_{V}<14,2$ mag, parece uma condição razoável a imposição de $m_{V}<14,2$ mag para classificar uma estrela como candidata a ser de foreground. Com esse limite possivelmente estaríamos excluindo apenas as estrelas frias e anãs pertencentes à VL. Procurando "driblar" o problema em classificar estrelas pertencentes à PNM como sendo de foreground, pode-se empregar um segundo filtro que exclui estrelas com polarizações muito diferentes das médias. Com isso, ainda que estrelas da PNM sejam consideradas como da VL, serão aquelas com polarização interestelar nula ou muito pequena. Esse segundo filtro também consegue eliminar possíveis estrelas que provavelmente pertençam à VL e sejam dotadas de polarização intrínseca e não apenas interestelar.

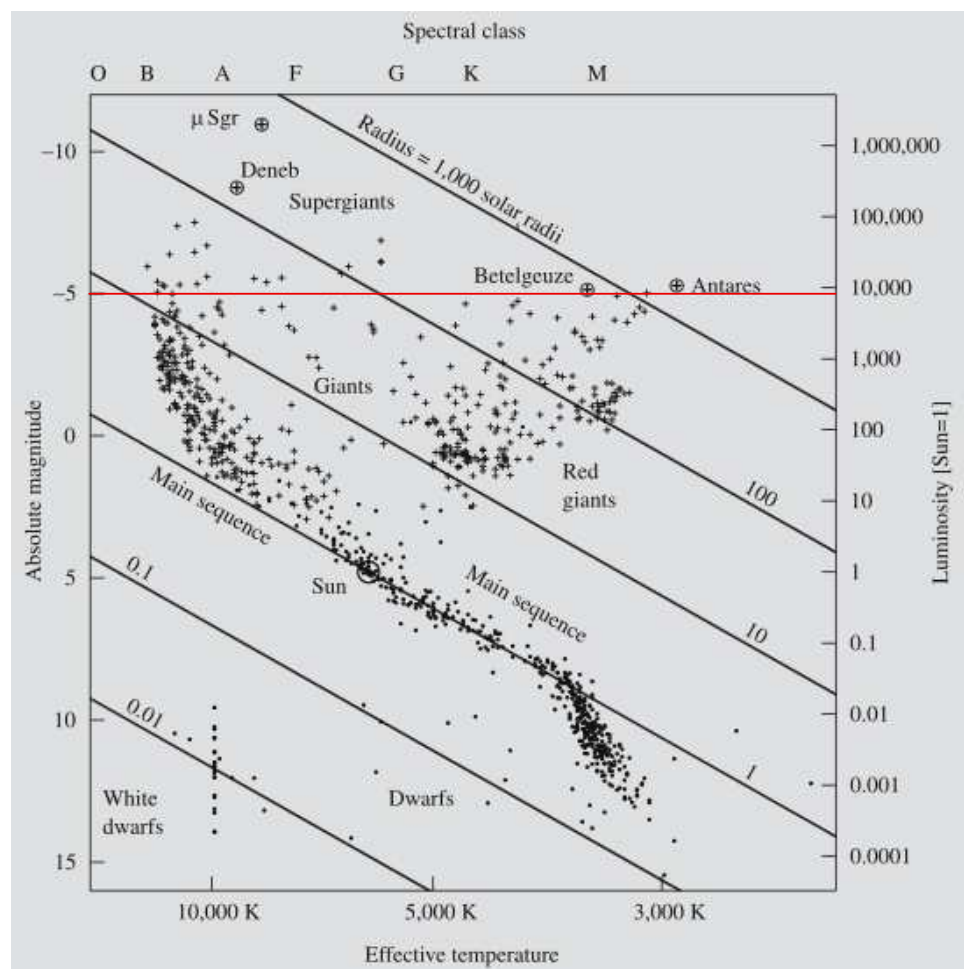

Figura 3.2: Diagrama HR retirado de Karttunen et al. (2007). A linha em vermelho separa as estrelas que possuem magnitudes absolutas maiores e menores do que -5 mag.

Com o critério de seleção definido, o próximo passo foi separar para cada campo quais são as estrelas candidatas a serem de foreground. Em seguida obtendo a polarização média e seu ângulo para cada região utilizando todas as estrelas pertencentes a ela. Para esses passos, foi escrito um script em IDL que realiza o seguinte procedimento:

1. Verifica as estrelas de cada campo que têm $m_{V}<14,2$ mag e $P<1,0 \%$. A polarização não pode ser maior do que $1 \%$ para evitar a consideração de estrelas que 
possam ter polarização intrínseca e não apenas interestelar.

2. Obtém e guarda os parâmetros de Stokes e seu erro para cada estrela selecionada

$$
\left\{\begin{array}{l}
Q=P \cos (2 \theta), \\
U=P \cos (2 \theta), \\
\sigma_{Q, U}=\sigma_{P}{ }^{12} .
\end{array}\right.
$$

3. Após verificar todas as estrelas de uma região, obtém os parâmetros de Stokes médios calculando uma média ponderada pela incerteza. Depois, confere quais estrelas possuem parâmetros Q e U dentro de $2 \sigma$ de distância da média calculada. Então, utilizando as $N$ estrelas que satisfazem esse critério, calcula novamente uma média ponderada pela incerteza

$$
\left\{\begin{array}{l}
\langle Q\rangle=\sum_{n=1}^{N}\left(\frac{Q_{n} / \sigma_{Q_{n}}^{2}}{1 / \sigma_{Q_{n}}^{2}}\right), \\
\langle U\rangle=\sum_{n=1}^{N}\left(\frac{U_{n} / \sigma_{U_{n}}^{2}}{1 / \sigma_{U_{n}}^{2}}\right), \\
\sigma_{\langle Q\rangle,\langle U\rangle}=\frac{\left[\sigma\left(Q_{n}\right)+\sigma\left(U_{n}\right)\right]}{2 \sqrt{N}} .
\end{array}\right.
$$

Considera-se a incerteza como o desvio padrão da média médio para os parâmetros Q e U. Não é considerada a incerteza da média ponderada pela incerteza, pois ela costuma ser subestimada.

4. Por fim, calcula a polarização de foreground média e seu ângulo para cada campo

$$
\left\{\begin{array}{l}
P_{\text {for, },}=\sqrt{\langle Q\rangle^{2}+\langle U\rangle^{2}} \\
\theta_{\text {for, } g}=\frac{1}{2} \operatorname{arctg}\left(\frac{\langle U\rangle}{\langle Q\rangle}\right) \\
\sigma_{P_{\text {for }, \mathrm{g}}}=\sigma_{\langle Q\rangle,\langle U\rangle}
\end{array}\right.
$$

Os gráficos das figuras 3.3 e 3.4 apresentam os parâmetros de Stokes de cada estrela selecionada como candidata a ser de foreground para cada região. A linha em vermelho

\footnotetext{
12 Pode-se mostrar, a partir da propagação de incertezas, que a incerteza da polarização é igual à incerteza dos parâmetros de Stokes, para o caso em que $\sigma_{Q}=\sigma_{U}$.
} 
equivale à média ponderada pela incerteza considerando todas essas estrelas, e as linhas em azul a $\pm 2 \sigma$. Assim, é possível observar que a utilização desse corte de fato exclui estrelas com parâmetros de Stokes que difiram muito dos valores médios. Esse segundo filtro é uma forma de cortar estrelas com provável polarização intrínseca ou que possam ser pertencentes à PNM e estar sofrendo polarização interestelar.
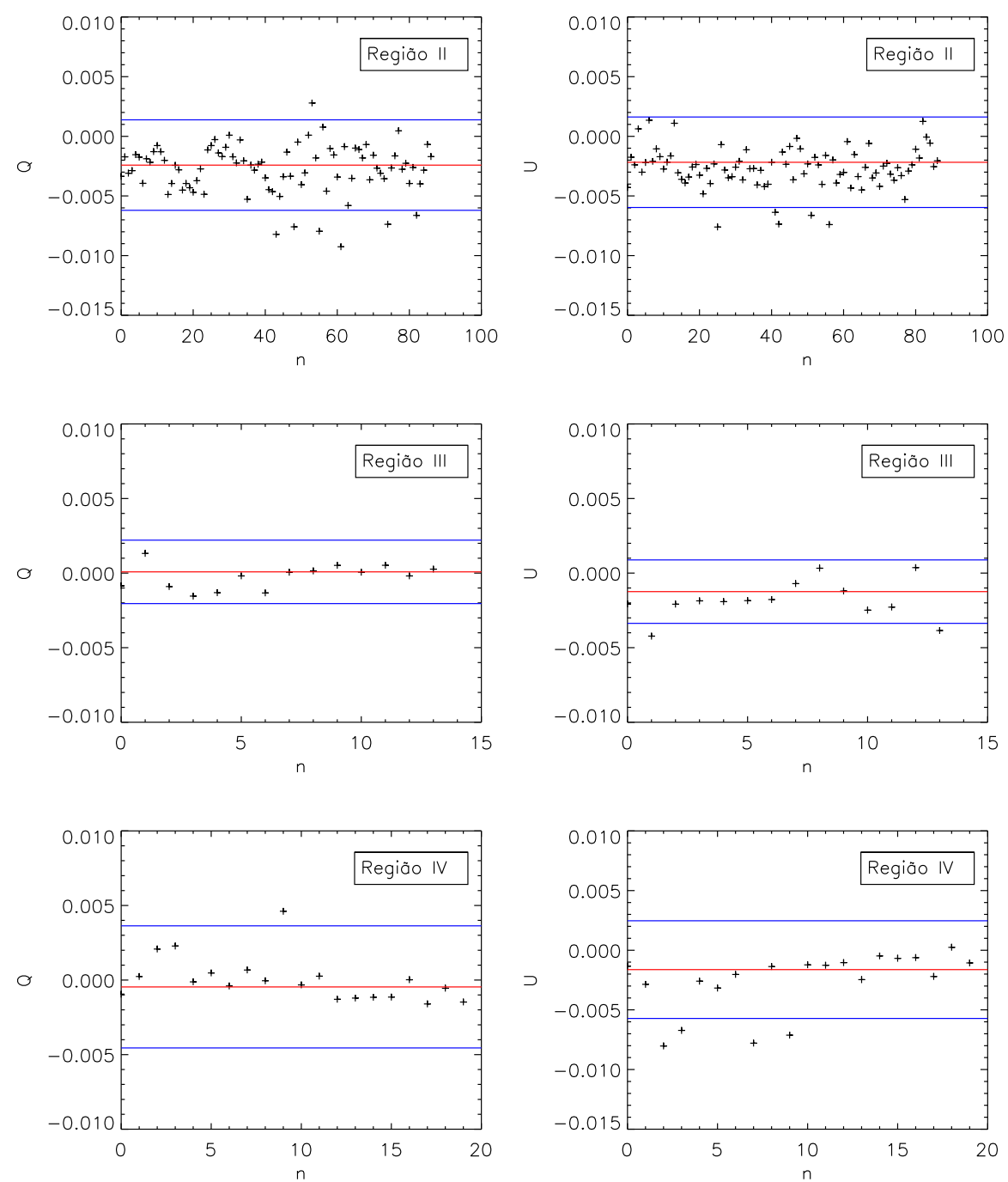

Figura 3.3: Gráficos por região dos parâmetros de Stokes Q e U das estrelas selecionadas como candidatas a serem de foreground. A linha em vermelho apresenta a média ponderada pela incerteza de cada parâmetro, e as linhas em azul $\pm 2 \sigma$.

A tabela 3.2 apresenta as polarizações de foreground e seus ângulos, obtidas a partir desse método e as estimadas por Rodrigues et al. (1997) e Schmidt (1976). 

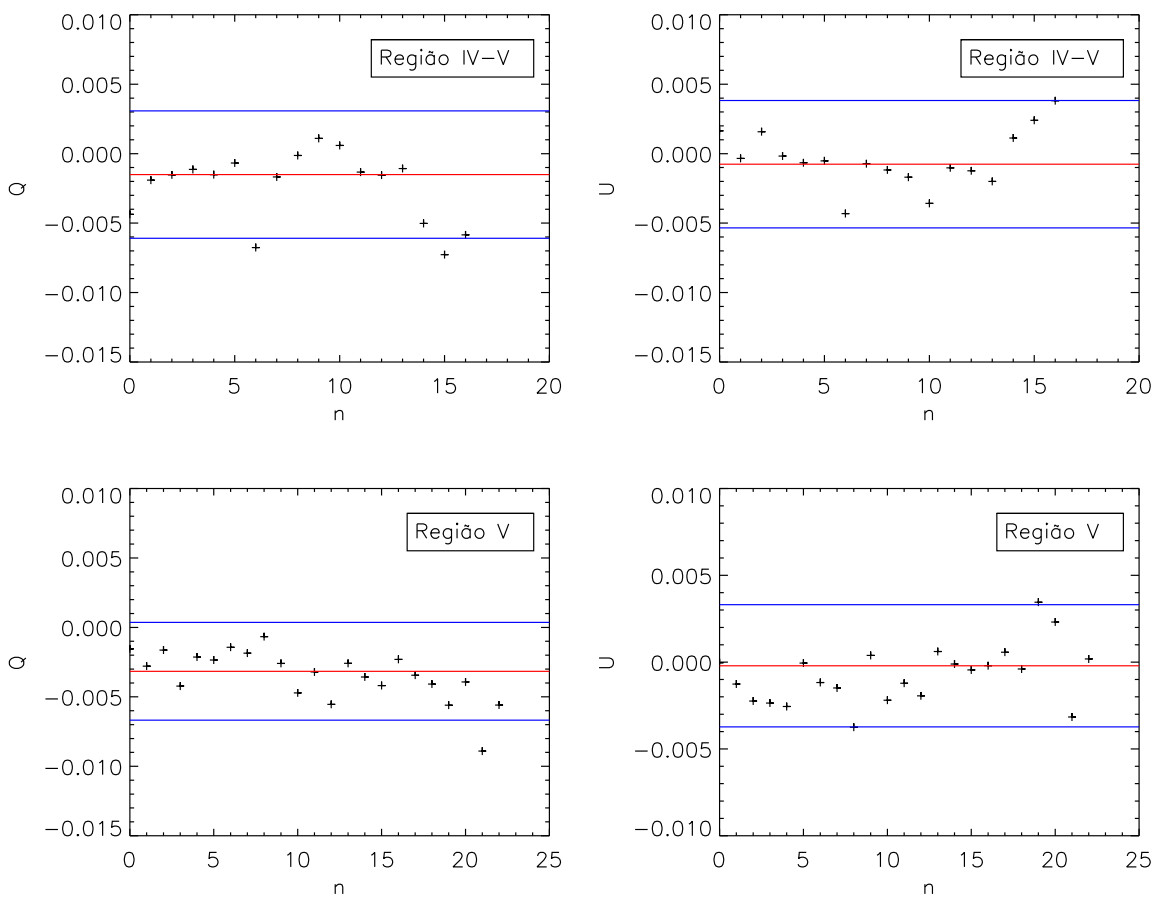

Figura 3.4: Gráficos por região dos parâmetros de Stokes Q e U das estrelas selecionadas como candidatas a serem de foreground. A linha em vermelho apresenta a média ponderada pela incerteza de cada parâmetro, e as linhas em azul $\pm 2 \sigma$ (continuação).

Tabela 3.2 - Estimativas da polarização de foreground para cada região da PNM. ( $P_{\text {for,s }}$ e $\left.\theta_{\text {for,s }}\right)$ são as polarizações obtidas por Schmidt (1976), ( $P_{\text {for,r }}$ e $\left.\theta_{\text {for,r }}\right)$ as obtidas por Rodrigues et al. (1997) e $\left(P_{\text {for,g }}, \theta_{\text {for }, \mathrm{g}}\right.$ e $\left.N_{\text {for }, g}\right)$ as obtidas neste trabalho e o número de estrelas utilizadas para estimá-la.

\begin{tabular}{|c|c|c|c|c|c|c|c|}
\hline Região & $P_{\text {for }, \mathrm{s}}(\%)$ & $\theta_{\text {for,s }}$ (graus) & $P_{\text {for }, \mathrm{r}}(\%)$ & $\theta_{\text {for,r }}$ (graus) & $P_{\text {for,g }}(\%)$ & $\theta_{\text {for,g }}$ (graus) & $N_{\text {for }, g}$ \\
\hline I & $(0,37 \pm 0,15)$ & 111 & $(0,47 \pm 0,09)$ & 113,6 & - & - & - \\
\hline II & $(0,27 \pm 0,15)$ & 123 & $(0,30 \pm 0,08)$ & 124,1 & $(0,316 \pm 0,016)$ & 111,6 & 75 \\
\hline III & $(0,06 \pm 0,09)$ & 139 & $(0,17 \pm 0,05)$ & 145,0 & $(0,124 \pm 0,024)$ & 136,8 & 12 \\
\hline IV & $(0,14 \pm 0,12)$ & 125 & $(0,22 \pm 0,05)$ & 124,2 & $(0,162 \pm 0,020)$ & 125,6 & 16 \\
\hline IV-V & - & - & - & - & $(0,138 \pm 0,048)$ & 102,7 & 15 \\
\hline V & $(0,16 \pm 0,12)$ & 93 & $(0,27 \pm 0,05)$ & 95,6 & $(0,301 \pm 0,028)$ & 91,1 & 20 \\
\hline
\end{tabular}

Ao comparar os valores obtidos por esse método e os previamente estimados por Rodrigues et al. (1997) e Schmidt (1976), nota-se que eles são compatíveis. Portanto, o método se mostrou satisfatório, e essas novas estimativas serão utilizadas para a subtração dessa polarização Galáctica. 


\subsubsection{Subtração da Polarização de Foreground}

Uma vez que determinamos a polarização de foreground para todas as regiões, o próximo passo foi eliminá-la para as estrelas dos catálogos. Para tanto, foi levado em consideração o fato de os parâmetros de Stokes serem aditivos. Assim, para eliminar essa polarização basta subtrair os parâmetros Q e U de foreground dos parâmetros observados, como a seguir

$$
\left\{\begin{array}{l}
Q_{c o r}=Q_{o b s}-Q_{\mathrm{for}}, \\
U_{c o r}=U_{o b s}-U_{\mathrm{for}}, \\
\sigma_{Q_{c o r}, U_{c o r}}=\sqrt{\sigma_{Q_{o b s}, U_{o b s}}^{2}+\sigma_{Q_{\mathrm{for}}, U_{\mathrm{for}}}^{2}},
\end{array}\right.
$$

onde $Q_{c o r}$ e $U_{c o r}$ são os parâmetros de Stokes corrigidos; $Q_{o b s}$ e $U_{o b s}$ são os parâmetros observados; $Q_{\text {for }}$ e $U_{\text {for }}$ são os parâmetros de foreground; $\sigma_{Q_{c o r}, U_{c o r}}$ é a incerteza para os parâmetros corrigidos; $\sigma_{Q_{o b s}, U_{o b s}}$ é a incerteza para os parâmetros observados; e $\sigma_{Q_{\text {for }}, U_{\text {for }}}$ é a incerteza para os parâmetros de foreground.

Essa correção foi realizada considerando as três diferentes estimativas de foreground este trabalho, Rodrigues et al. (1997) e Schmidt (1976). Para o caso das duas últimas, os campos que não se encaixavam em nenhuma região foram desprezados. Foi escrito um script em IDL para realizar essa subtração, o qual levava em conta a qual região cada estrela pertencia. Ele também desconsiderava as estrelas que foram selecionadas como sendo de foreground.

Após realizar a subtração, foram gerados cinco catálogos finais - o primeiro com as estrelas dos 28 campos e polarizações observadas; o segundo com as polarizações corrigidas das estrelas que são pertencentes à PNM, e utilizando a correção de foreground deste trabalho; o terceiro com as polarizações corrigidas das estrelas que são pertencentes à PNM, que se encaixavam em alguma região, e utilizando a correção de foreground de Rodrigues et al. (1997); o quarto com as polarizações corrigidas das estrelas que são pertencentes à PNM, que se encaixavam em alguma região, e utilizando a correção de foreground de Schmidt (1976); e o quinto com as estrelas candidatas a serem de foreground. Esses catálogos podem ser encontrados no endereço http://www. astro.iag. usp.br/ polarimetria/survey/.

A fim de analisar o efeito que a subtração do foreground causa nos dados, construímos histogramas para $P_{c o r} / P_{o b s}$ e $\theta_{c o r}-\theta_{o b s}$. Estes gráficos estão apresentados na figura 3.5. 

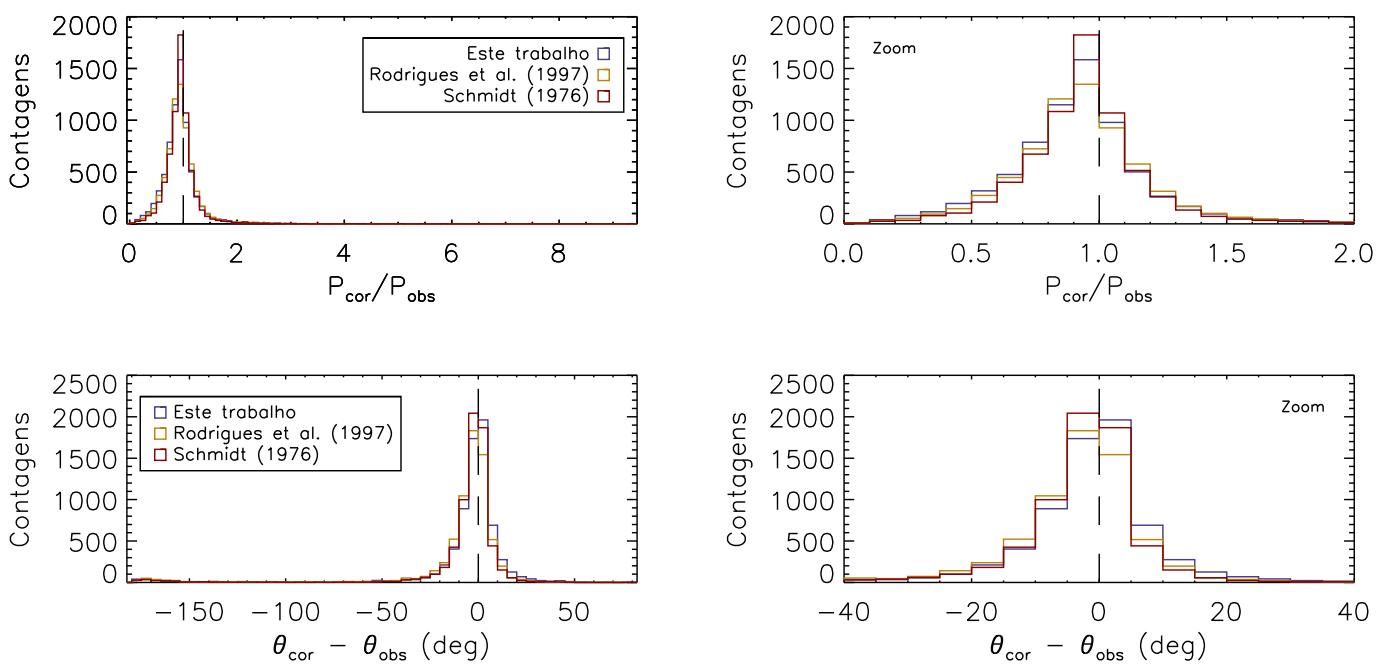

Figura 3.5: Histogramas para razão entre as polarizações corrigidas e observadas $\left(P_{c o r} / P_{o b s}\right)$ e diferença entre os ângulos de polarização corrigidos e observados $\left(\theta_{\text {cor }}-\theta_{\text {obs }}\right)$. Os histogramas em azul representam os dados com a correção de foreground obtida neste trabalho, em laranja a correção de Rodrigues et al. (1997) e em vermelho a correção de Schmidt (1976). As linhas pontilhadas representam o caso hipotético onde a correção de foreground é nula. Os histogramas da direita correspondem a um zoom para melhor visualização dos resultados.

Nota-se, pela análise dos histogramas, que a subtração da polarização Galáctica modifica o grau de polarização em até cerca de $50 \%$ do valor observado. Como era de se esperar, para a maior parte das estrelas essa subtração reduz a intensidade da polarização. Porém, para alguns casos, ela aumenta a sua intensidade. Esse fato parece estranho, mas pode ser explicado pelo caráter vetorial da polarização. Para casos específicos, por exemplo vetores em direções opostas, a soma da componente da polarização de foreground pode diminuir os parâmetros de Stokes, levando a um decréscimo para a polarização observada. Com relação ao ângulo de polarização, percebe-se que ele se modifica em até aproximadamente 20 graus, para aumento ou diminuição.

De forma global, não se verifica grande diferença entre as distintas correções de foreground utilizadas. Os histogramas para cada caso são bastante semelhantes. Apenas constata-se que a correção de Schmidt (1976) possui picos mais intensos em torno do caso em que a correção seria nula. Por outro lado, a correção deste trabalho possui picos menos intensos nessa região. Portanto, a correção deste trabalho tende a modificar mais a geometria observada para a polarização. 


\subsection{Análise do Catálogo Polarimétrico}

Possuindo o catálogo polarimétrico, com magnitudes calibradas e polarizações corrigidas, o próximo passo de interesse foi analisar a distribuição de magnitudes e estudar se existe alguma relação entre polarização e magnitude.

\subsubsection{Magnitudes na Banda $V$}

Para estudar a distribuição de magnitudes foi feito um histograma desta, considerando todas as estrelas do catálogo, o qual pode ser visualizado na figura 3.6. A fração de estrelas de foreground é baixa quando comparada ao número total de estrelas do catálogo, apenas 2,3\% das estrelas são candidatas a pertencerem à Galáxia. Com relação às estrelas pertencentes à PNM, o pico do histograma acontece para $m_{V}=18$ mag, porém existe um número razoável de estrelas que pertencem à faixa de 19 mag. Logo, pode-se dizer que as observações foram capazes de alcançar estrelas de magnitudes aparentes na banda $\mathrm{V}$ de até aproximadamente 19 mag.

Foram construídos histogramas para magnitude separando as estrelas por regiões, os quais também podem ser visualizados na figura 3.6. De modo geral a distribuição de magnitudes por região são semelhantes. Contudo, percebe-se que a região II é a mais rica em estrelas, ela está localizada na Barra da PNM.

Em seguida, as regiões III e IV formam a Asa da PNM. Nota-se que o número de estrelas vai diminuindo conforme se afasta da Barra, e o número de estrelas com magnitudes próximas de $m_{V}=19$ mag vai crescendo com relação a $m_{V}=18 \mathrm{mag}$, e na região IV o pico do histograma modifica-se para aquela. O número de estrelas que pertencem à faixa $m_{V}=20$ mag também vai crescendo com relação aos demais conforme se afasta da Barra.

Nas regiões IV-V e V o número de estrelas diminui ainda mais. E esse é o início da Ponte pan-Magelânica. Na região $\mathrm{V}$, o número de estrelas com magnitude $m_{V}=17$ mag se torna comparável ao de magnitudes 18 e 19. A população estelar da Ponte aparenta ser mais rica em estrelas brilhantes, o que indica uma população mais jovem. 

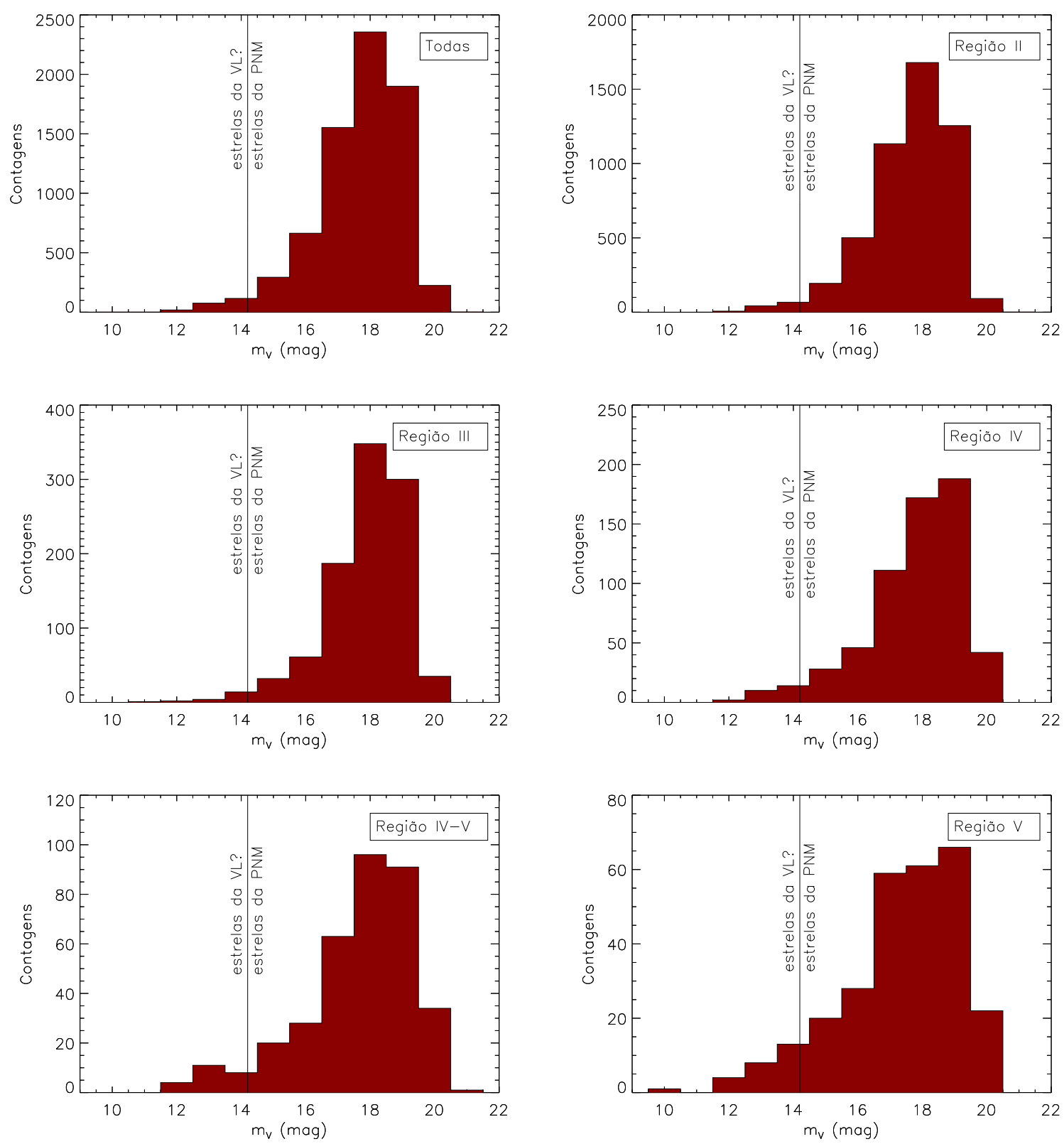

Figura 3.6: Histogramas para as magnitudes na banda V, considerando todas as estrelas e separando por regiões. A linha vertical, em $m_{V}=14,2$ mag, separa as estrelas candidatas a serem de foreground das que são admitidas como pertencentes à PNM. 


\subsubsection{Polarização versus Magnitude}

Uma segunda análise de interesse, no caso entre parâmetros das estrelas dos catálogos, é verificar se existe alguma relação entre a intensidade da polarização e a magnitude aparente na banda V. Para tanto, foram construídos gráficos de $P$ vs $m_{V}$ (figura 3.7), um utilizando todas as estrelas consideradas como pertencentes à PNM e outros separando essas estrelas por regiões para examinar se havia alguma mudança de comportamento. Vale ressaltar que foi considerada a polarização corrigida por foreground. Como essa correção levou ao aumento do erro nas polarizações medidas, foram utilizadas apenas estrelas com $P / \sigma_{P}>3$ para construção dos gráficos.

Pela comparação dos gráficos, nota-se que não houve mudança na relação entre polarização e magnitude para as diferentes regiões consideradas. Em todos os casos constata-se um aumento de $P$ com $m_{V}$. Se levarmos em conta que, em média, as estrelas com magnitudes aparentes altas se encontram mais distantes, esse aumento da polarização pode ser facilmente justificado. A luz das estrelas mais distantes precisa atravessar uma camada maior de poeira, podendo então sofrer uma maior polarização interestelar.

Apesar desse aumento ser facilmente visível, não é possível dizer qual a forma da relação, a dispersão dos pontos é bem alta, e também nota-se um acúmulo de estrelas com polarização baixa para toda a faixa de magnitudes. Esse comportamento pode acontecer devido a uma distribuição homogênea de tipos espectrais com a distância. Pode haver estrelas fracas (frias e anãs) e próximas, e portanto com polarização em média baixa, levando ao acúmulo observado na parte inferior dos gráficos. Por outro lado, podem existir estrelas brilhantes (quentes) e distantes, possuindo em média polarizações mais altas. Por isso, são observadas estrelas com polarizações um pouco altas para baixos valores de $m_{V}$. Mais do que isso, deve haver objetos com polarização intrínseca, a qual depois é somada à polarização interestelar, atrapalhando o estudo da relação do aumento de $P$ com a distância. 

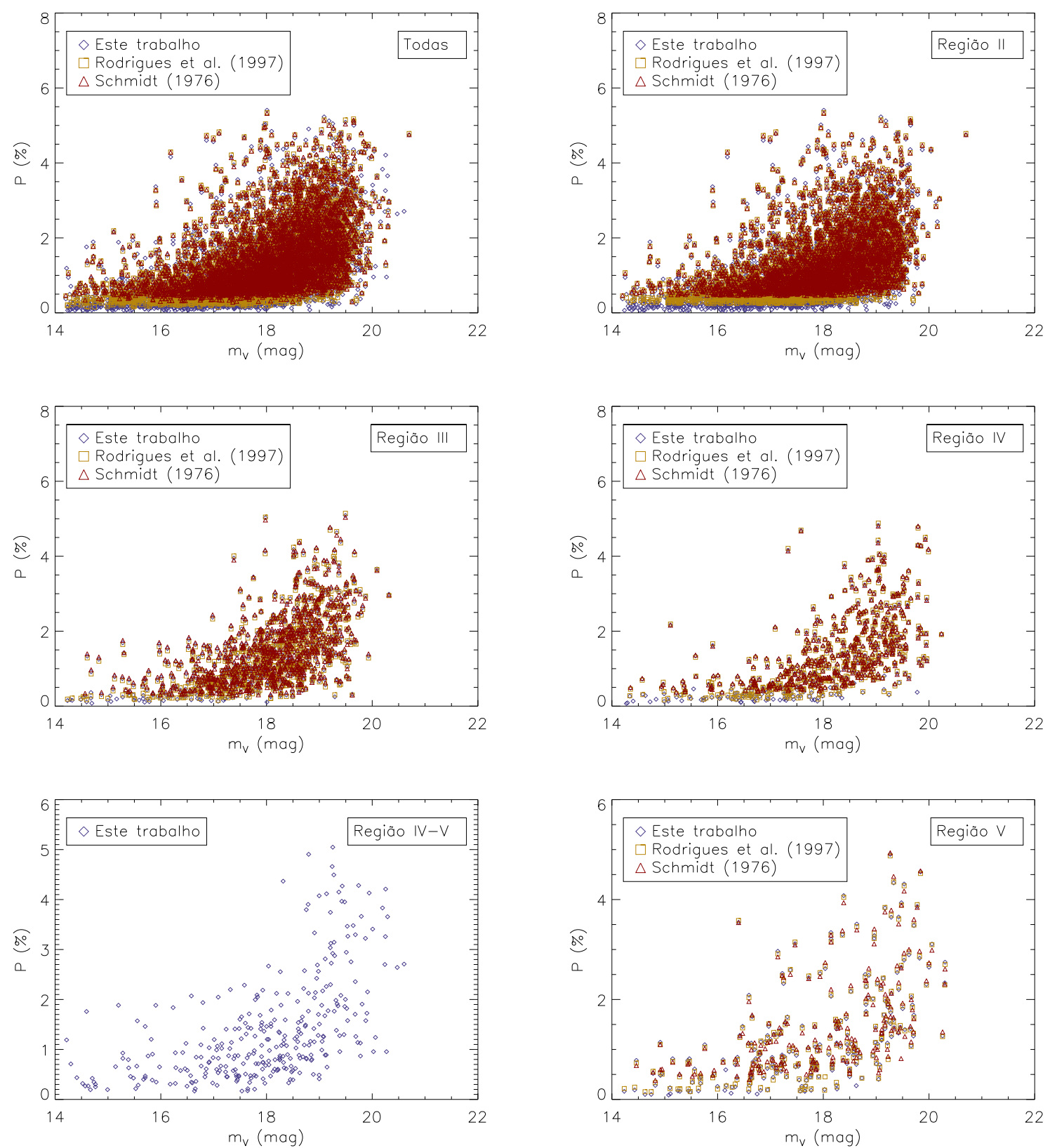

Figura 3.7: Gráficos da polarização versus magnitude, considerando todas as estrelas e separando por regiões. Os diamantes azuis são os dados com polarização corrigida por foreground estimado neste trabalho, os quadrados laranjas considerando a correção de Rodrigues et al. (1997) e os triângulos vermelhos considerando a correção de Schmidt (1976). 


\subsection{Mapa de Polarização}

O próximo passo foi analisar os padrões de polarização para cada campo. Para tanto, construímos histogramas para o ângulo de polarização $(\theta)$. Novamente, utilizamos apenas as estrelas que ficaram com $P / \sigma_{P}>3$ após a subtração do foreground, e foi considerado o catálogo que empregou a correção de foreground estimada neste trabalho. A fim de obter o pico para o padrão de $\theta$ e o seu desvio padrão, ajustamos gaussianas para os histogramas.

Para conhecer a distribuição da intensidade da polarização $(P)$ para cada padrão, construímos histogramas para $P$, apenas com as estrelas que possuíam ângulos de polarização dentro de $3 \sigma$ do valor médio de $\theta$. Por fim, utilizamos a mediana dos valores de $P$ dessas estrelas para caracterizar como polarização do padrão principal. Todo esse procedimento foi automatizado em IDL.

Notamos distribuições distintas para $\theta$ considerando os 28 campos observados. Assim, se fez necessário avaliar cada campo separadamente, com o intuito de obter uma melhor caracterização dos seus padrões. Abaixo está descrito como tratamos cada caso e quais campos se encaixam em cada um.

\subsubsection{Campos com Um Padrão}

Oito campos apresentaram apenas um padrão para o ângulo de polarização - PNM 03, 06, 09, 11, 15, 18, 21 e 28. Desse modo, ajustamos apenas uma gaussiana para esses histogramas. Como exemplo, três campos que se encaixam nessa categoria são expostos na figura 3.8.

O campo PNM06 apresenta um desvio padrão bastante alto. Mesmo assim, decidimos ajustar a gaussiana para ele, pois não podíamos assumir que a distribuição era aleatória. O campo PNM09 possui um padrão bastante acentuado, e quase é possível dizer que existe um segundo padrão. Porém, como ele não tem uma contagem de estrelas com um valor razoável, acima do "fundo aleatório", decidiu-se não ajustar uma segunda gaussiana nele. Finalmente, o campo PNM28 é um exemplo de um campo que apresenta apenas um padrão e aparenta ser único. Os gráficos para os outros campos podem ser visualizados no apêndice A. 

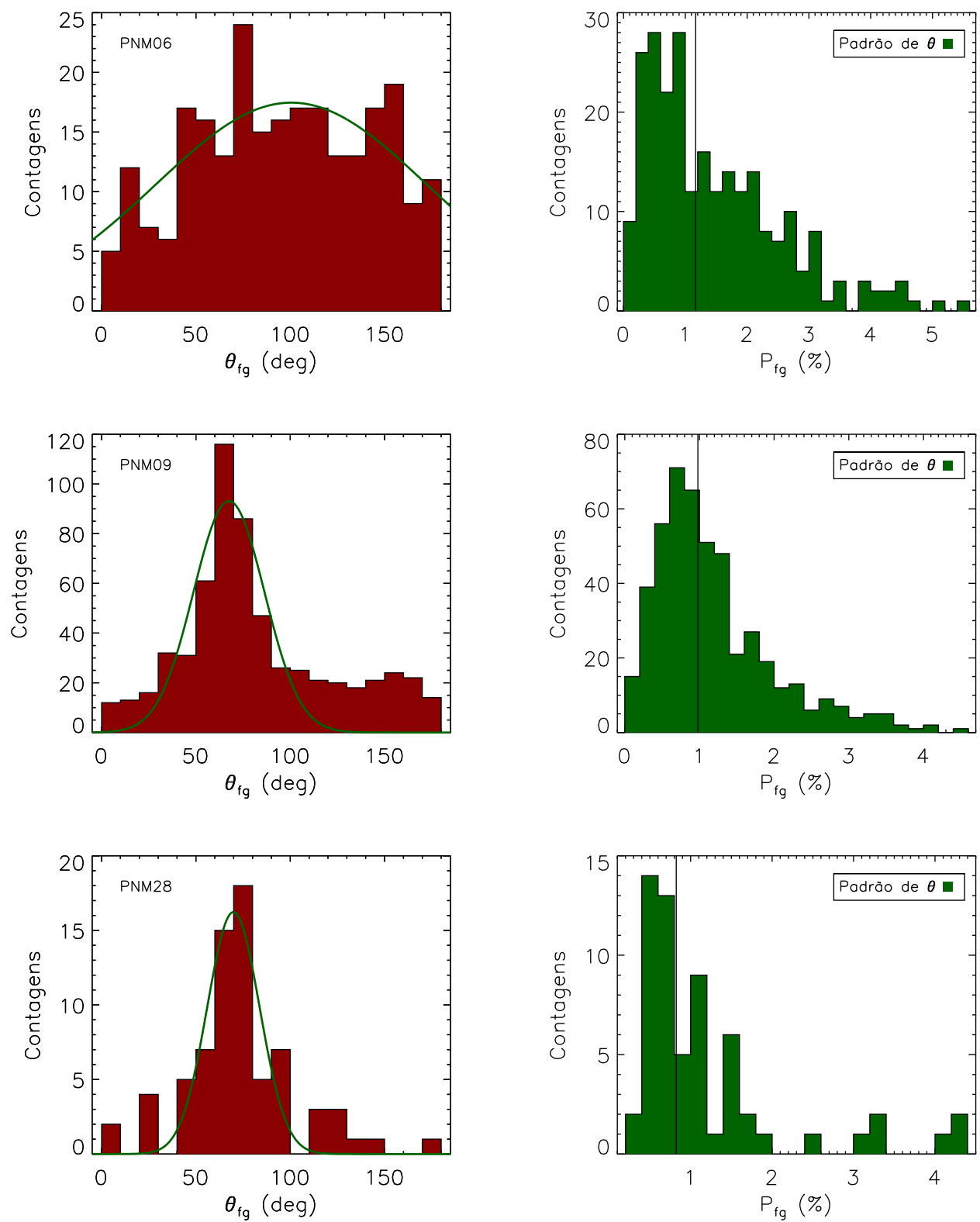

Figura 3.8: Exemplos de campos com apenas um padrão para $\theta$. Histogramas para o ângulo de polarização e a sua intensidade. A linha vertical no histograma de $P$ marca a sua mediana. 


\subsubsection{Campos com Dois Padrões}

Oito campos apresentaram dois padrões para $\theta$ - PNM 01, 02, 04, 07, 08, 10, 22 e 27. Desse modo, ajustamos duas gaussianas aos seus histogramas. Para verificar a distribuição da polarização de cada padrão, utilizou-se as estrelas com ângulos dentro de $3 \sigma$ do valor médio de $\theta$ de cada padrão. Assim, é possível que a soma das contagens dos dois histogramas de $P$ seja superior ao número total de estrelas no campo, já que podem haver estrelas pertencentes aos dois padrões.

O gráfico da figura 3.9 expõe como exemplo o caso do campo PNM01. Ao redor de $\theta_{f g} \sim 0$ graus, há uma concentração de estrelas que parece indicar um terceiro padrão. Em outros campos a existência de um terceiro padrão podia ser esperada. Contudo, decidimos ajustar apenas padrões que se sobressaiam do "fundo aleatório", e que de fato aparentavam estar separados, quer dizer, seus picos possuíam um número razoável de bins de distância. Os gráficos para os demais campos podem ser visualizados no apêndice A.
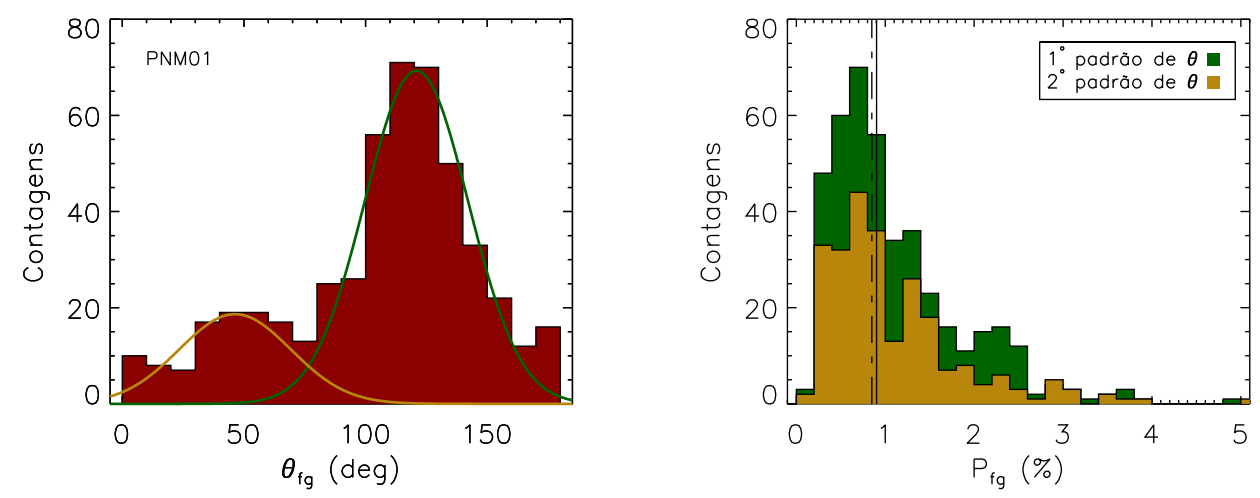

Figura 3.9: Exemplo de campo com dois padrões para $\theta$. Histogramas para o ângulo de polarização e a sua intensidade. As linhas verticais no histograma de $P$ marcam as medianas para o primeiro (linha sólida) e segundo padrão (linha sólida-pontilhada).

\subsubsection{Campos com Nenhum Padrão}

Quatro campos não apresentaram nenhum padrão para $\theta$ - PNM 05, 14, 19 e 25. Desse modo, logicamente não foi ajustada nenhuma gaussiana. Os gráficos da figura 3.10 mostram os histogramas para os campos PNM 05 e 14. Para o primeiro, pode-se notar quatro picos distintos, porém o número de estrelas não é suficiente para que se faça uma estatística confiável. Por isso foi considerado que ele não possui nenhum padrão. Em todo 
caso, é possível que haja um padrão nessa região com pico próximo de $\theta_{f g} \sim 100$ graus. O caso do segundo exemplo é mais extremo. De fato para essa região a distribuição dos ângulos parece aleatória. Os histogramas de polarização foram construídos com as mesmas estrelas dos histogramas de $\theta$. Os gráficos dos demais campos podem ser visualizados no apêndice A.
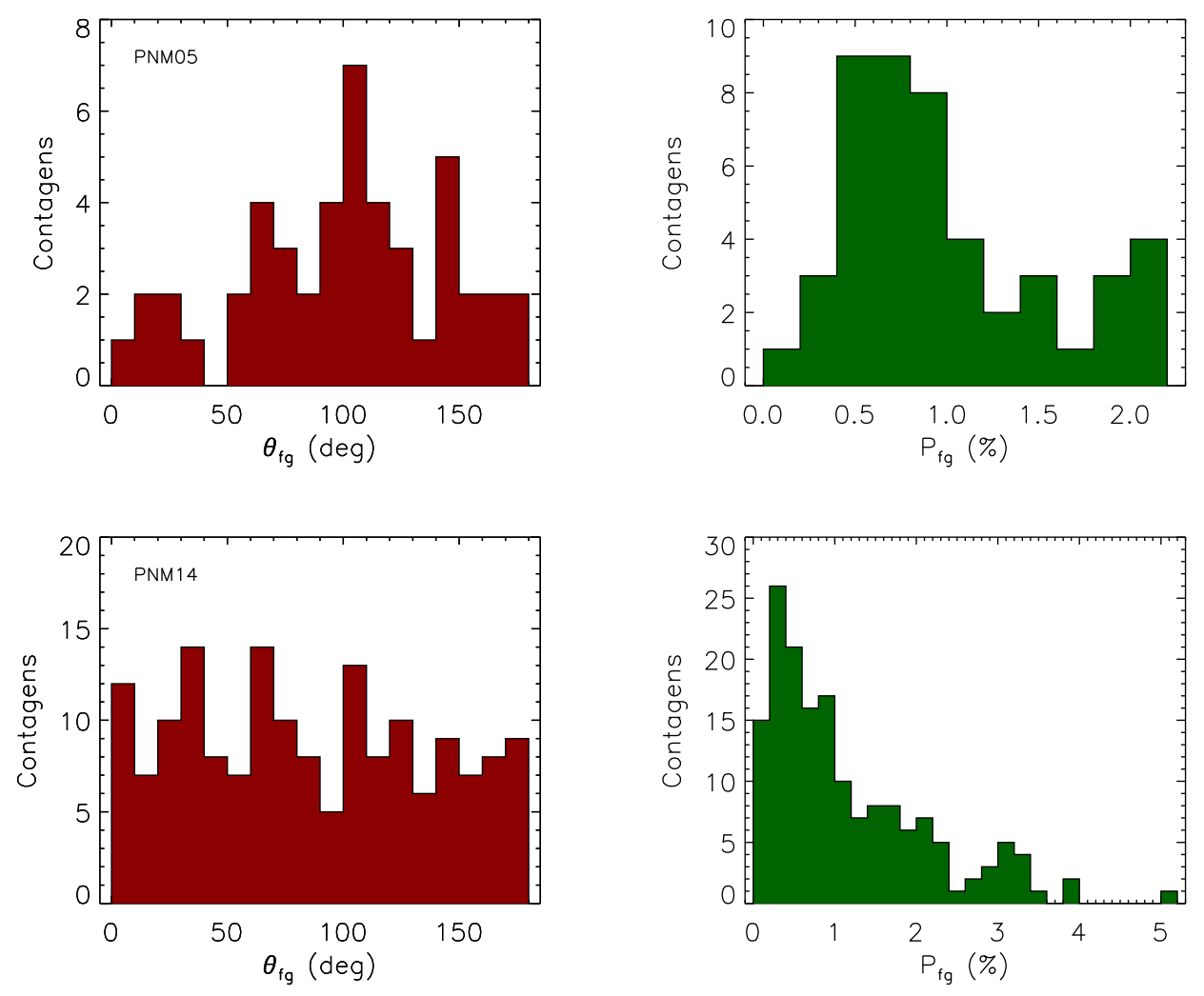

Figura 3.10: Exemplos de campos com nenhum padrão para $\theta$. Histogramas para o ângulo de polarização e a sua intensidade.

\subsubsection{Campos Filtrados por $m_{V}$}

Quatro campos apresentaram um fundo aleatório bastante acentuado - PNM 12, 13, 20 e 23, contudo era possível perceber a existência de um padrão. Devido a esse fundo, o ajuste das gaussianas era fortemente afetado. Verificou-se que, apesar de ser possível obter o valor do pico, os desvios padrões não pareciam realísticos. Porém, é importante que os desvios padrões sejam bem determinados para posterior estimativa da intensidade do campo magnético. 
Para tentar melhorar os ajustes nesses campos, construímos gráficos de $\theta$ em função de $m_{V}$, com o objetivo de examinar se esse fundo poderia ser separado do padrão observado. Os gráficos da figura 3.11 apresentam os histogramas para $\theta$ e $P$, assim como o gráfico de $\theta$ vs $m_{V}$ para o caso do campo PNM12. Nesse caso específico, notou-se que o padrão aleatório estava concentrado para as estrelas com $m_{V}>17,5$ mag. Então, foram utilizadas apenas as estrelas com magnitudes menores do que esse valor para a realização do ajuste. Esse procedimento melhorou consideravelmente os ajustes para esses campos. Os gráficos para os outros campos estão expostos no apêndice A.

Lembrando que as magnitudes aparentes podem ser utilizadas como indicadoras de distância. Para justificar a filtragem em $m_{V}$, podemos dizer que o padrão aleatório está separado em distância dos demais.
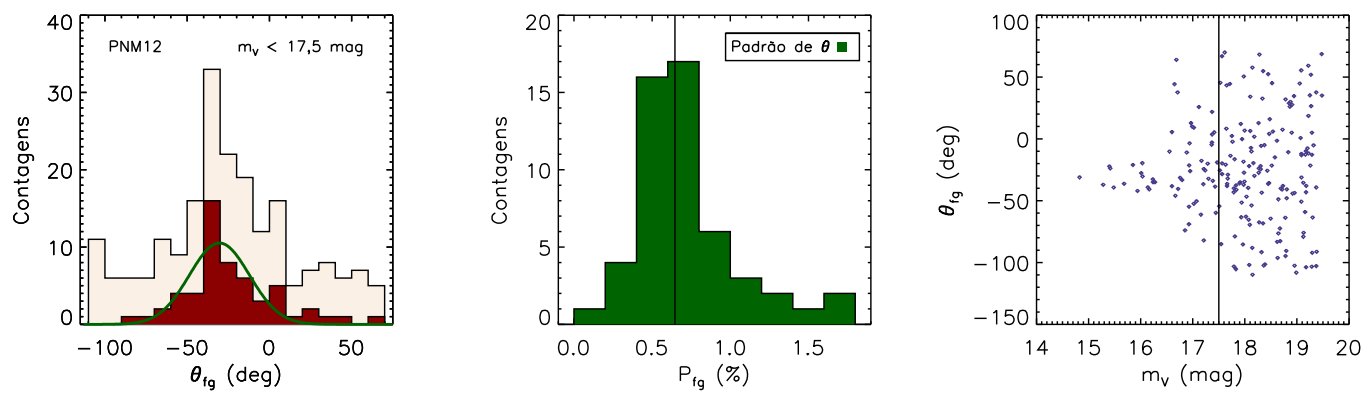

Figura 3.11: Exemplo de campo filtrado por $m_{V}$ para visualização do padrão de $\theta$. Histogramas para o ângulo de polarização e a sua intensidade, e gráfico de $\theta$ vs $m_{V}$. A linha vertical no histograma de $P$ marca a sua mediana, e no gráfico de $\theta$ vs $m_{V}$ destaca a magnitude de corte. O histograma em rosa apresenta as estrelas para toda a faixa de magnitudes.

\subsubsection{Campos com Estrelas Excluídas}

Um último caso que observamos foi o de quatro campos nos quais visualmente notavase que o ajuste não parecia razoável - PNM 16, 17, 24 e 26. No caso do campo PNM24, a partir do gráfico de $\theta$ vs $m_{V}$, percebeu-se a existência de dois padrões distintos para $\theta$. Um deles era mais acentuado, porém o segundo com uma baixa contagem de estrelas, de modo que o ajuste de duas gaussianas não seria confiável. Então, decidiu-se cortar as estrelas deste segundo padrão para efetuação do ajuste. Os gráficos correspondentes a esse campo podem ser visualizados na figura 3.12. Ela também apresenta os gráficos para o campo PNM16. Nesse caso e nos demais, não foi observado nenhum comportamento 
diferente para a faixa de magnitudes. Entretanto, para que fosse possível a realização do ajuste, decidimos retirar as estrelas correspondentes aos bins que pareciam influenciá-lo e dificultavam que o programa convergisse para uma solução razoável.
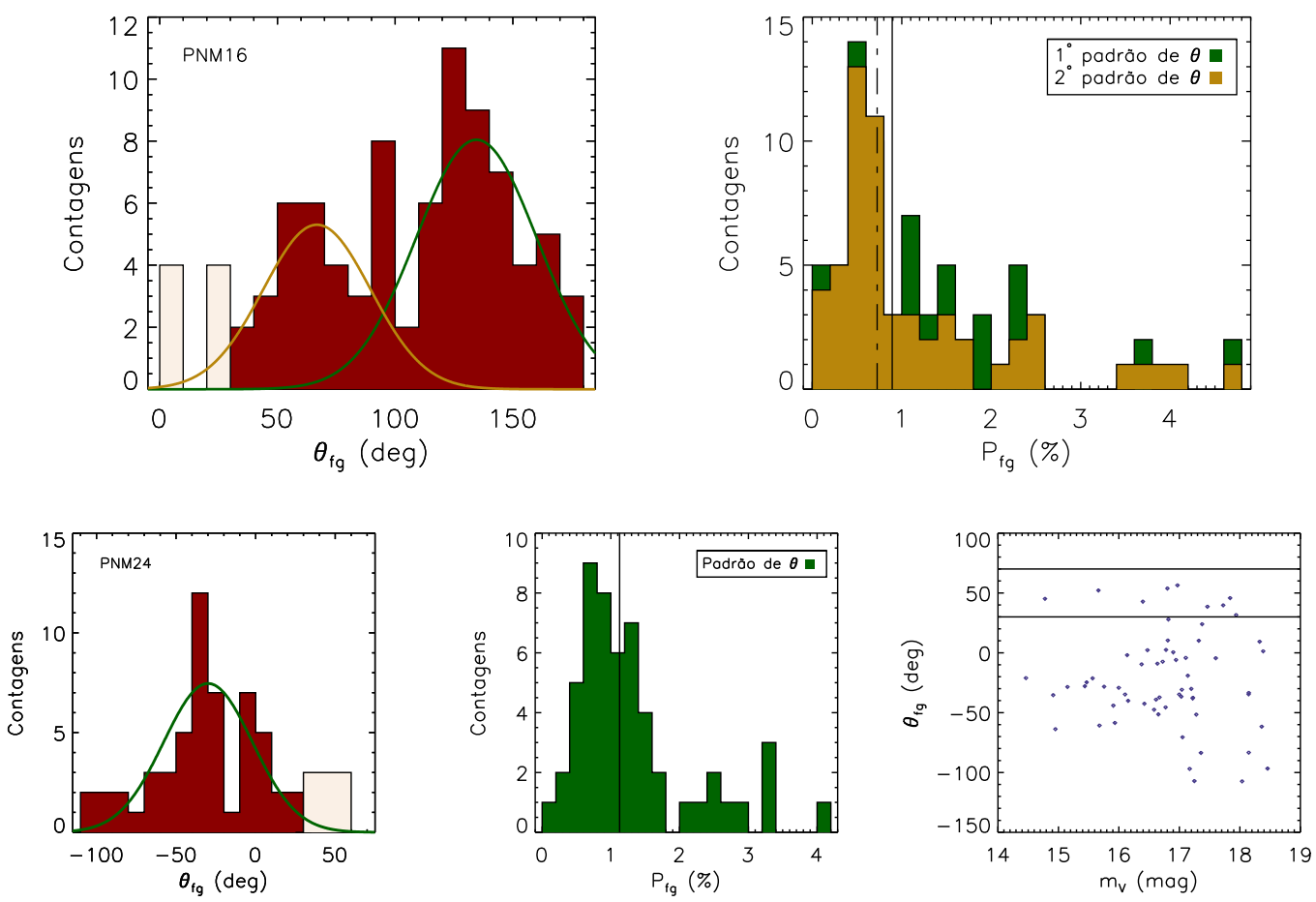

Figura 3.12: Exemplos de campos com estrelas excluídas para ajuste do padrão de $\theta$. Histogramas para o ângulo de polarização e a sua intensidade. As linhas verticais no histograma de $P$ marcam as medianas para o primeiro (linha sólida) e segundo padrão (linha sólidapontilhada). Os bins em rosa claro, no histograma de $\theta$, correspondem a estrelas que não foram consideradas no ajuste. Para o campo PNM24, também está apresentado o gráfico de $\theta$ vs $m_{V}$. As estrelas entre as linhas horizontais foram excluídas para efetuação do ajuste.

\subsubsection{Mapa de Polarização}

Construímos mapas utilizando os picos de $\theta$ e medianas de $P$. Como os vetores de polarização traçam o campo magnético no plano do céu, esse mapa pode ser interpretado como um mapa do campo magnético nessa direção.

Foram construídos dois mapas de polarização (figuras 3.13 e 3.14). Para o caso dos campos que possuem dois padrões de polarização, o primeiro mapa mostra o padrão com maior intensidade de polarização, e o segundo com menor. No apêndice A, tabela A.1, estão apresentados os valores dos picos de $\theta$ e medianas de $P$ que foram utilizados para a 
construção dos mapas.

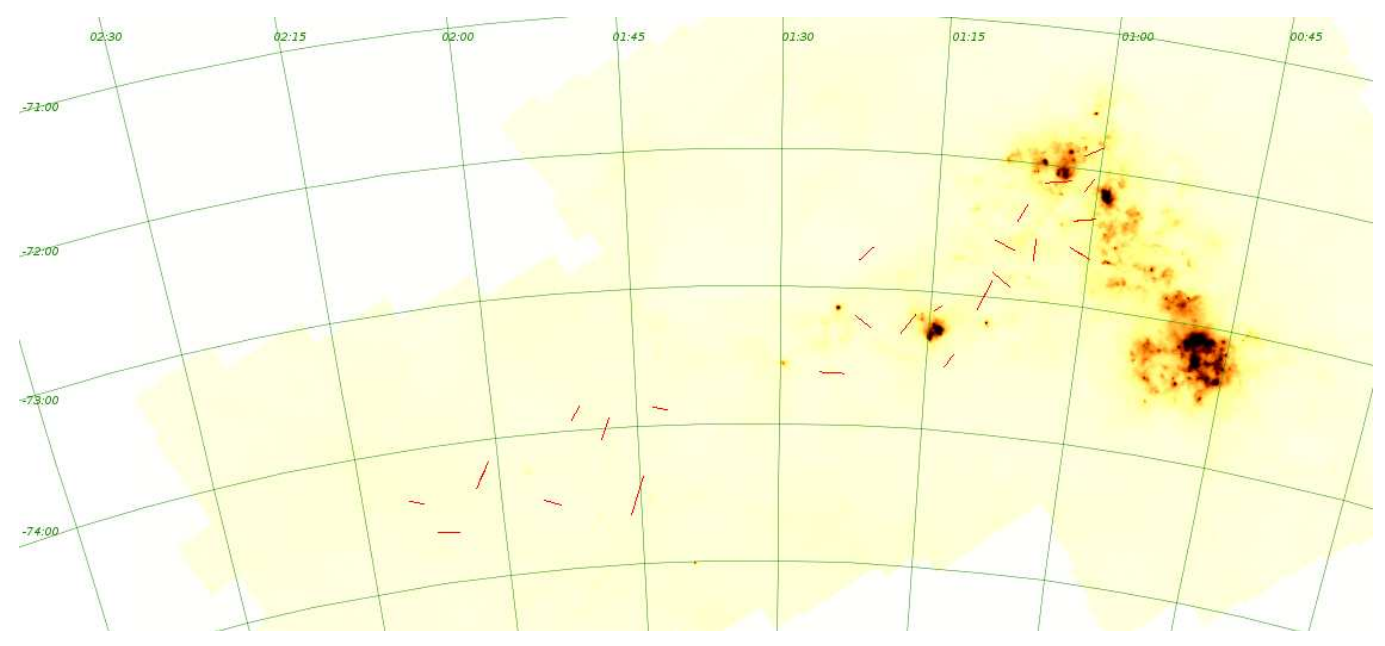

Figura 3.13: Mapa dos padrões de polarização. No caso de campos com dois padrões, está apresentado o padrão que corresponde à maior intensidade de polarização. Os vetores estão sobrepostos a uma imagem do Spitzer-MIPS em $160 \mu \mathrm{m}$.

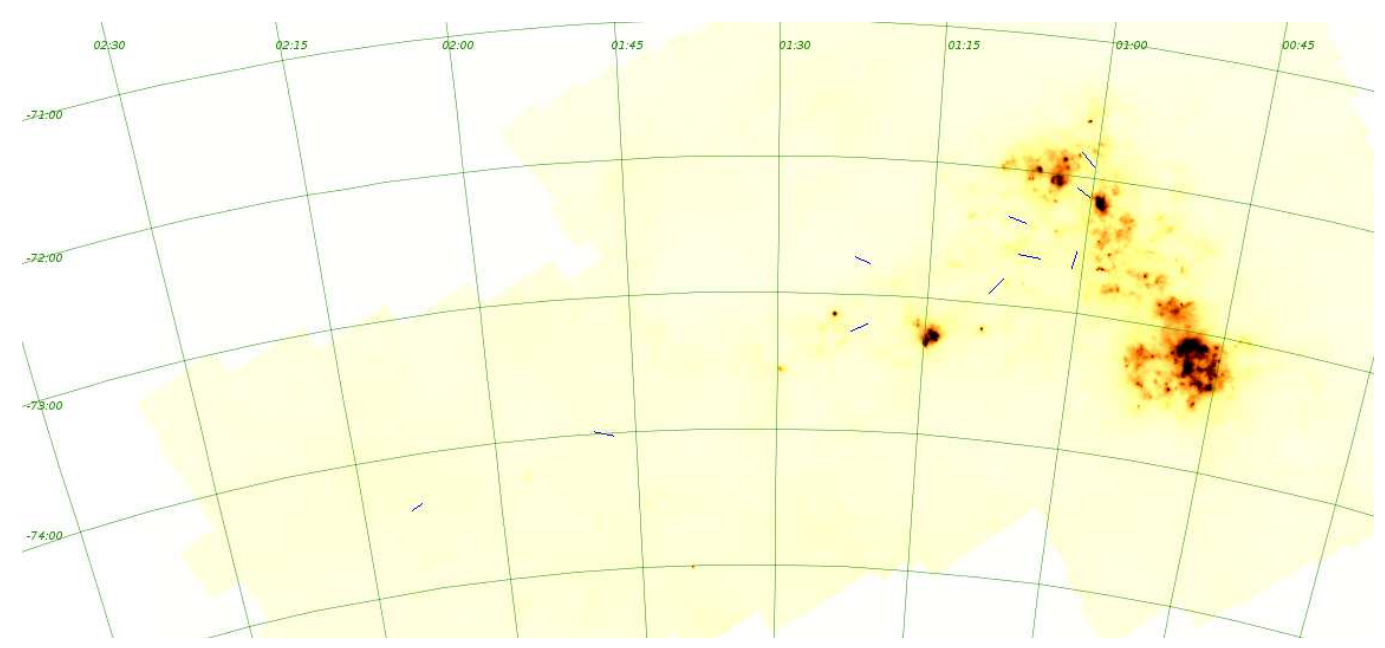

Figura 3.14: Mapa dos padrões que possuem menor intensidade de polarização, para os campos que apresentaram dois padrões. Os vetores estão sobrepostos a uma imagem do Spitzer-MIPS em $160 \mu \mathrm{m}$.

O mapa da figura 3.13 demonstra uma configuração bastante irregular para os vetores. Alguns desses padrões podem estar associados a estruturas específicas do meio interestelar da PNM, o que pode causar essa distribuição desordenada para os vetores. Na seção 4.1 procuramos associar esses padrões a objetos observados no MI da PNM.

O mapa da figura 3.14 possui um número menor de vetores, porém podemos supor a existência de dois padrões em larga escala - alinhado com a Barra (três vetores na 
região Nordeste) e alinhado com a direção da Ponte pan-Magelânica (cinco vetores na Asa e Ponte). Esse alinhamento, do campo magnético em larga escala da PNM com a Ponte, foi proposto nos anos 70 por Schmidt $(1976,1970)$ e Mathewson e Ford (1970b,a). Atualmente ele foi reestudado e confirmado por Magalhães et al. (2009, 1990) e Mao et al. (2008). No entanto, em todos os casos foi utilizada uma amostra pequena de estrelas e cujas polarizações foram determinadas através de dados fotoelétricos, ou seja, as precisões eram bem menores do que as obtidas com os dados CCD deste trabalho. Assim, agora podemos fazer uma análise mais acurada para esta suposição.

A fim de analisar esse possível alinhamento, primeiramente calculamos o ângulo $\left(\theta_{M}\right)$ entre os vetores de polarização de cada estrela e a direção do centro da Grande Nuvem de Magalhães, que também indica a direção da Ponte. Foi utilizado como centro da GNM a posição com $\mathrm{AR}(\mathrm{J} 2000)=05^{h} 23^{m} 34.6^{s}$ e $\mathrm{DEC}(\mathrm{J} 2000)=-69^{\circ} 45^{\prime} 22^{\prime}$. Posteriormente, construímos histogramas para os ângulos $\theta_{M}$ para quatro casos - polarização observada $\left(\theta_{M, o b s}\right)$, polarização corrigida por foreground de Schmidt (1976) $\left(\theta_{M, f s}\right)$, corrigida por foreground de Rodrigues et al. (1997) $\left(\theta_{M, f r}\right)$ e corrigida por foreground deste trabalho $\left(\theta_{M, f g}\right)$. Esses gráficos podem ser visualizados na figura 3.15.

Nota-se que o histograma para polarização observada apresenta um pico bem acentuado em 0 graus, o que indicaria campo magnético alinhado com a Ponte. Porém, em todos os casos em que a polarização de foreground é subtraída, esse pico some. Os histogramas para polarizações corrigidas por foreground de Rodrigues et al. (1997) e Schmidt (1976) possuem um pico não muito acentuado em -40 graus. Mas, ao utilizar a correção deste trabalho, o histograma apresenta uma distribuição mais próxima de aleatória. Esse resultado leva a crer que o alinhamento proposto, entre o campo magnético e a Ponte, pode ser um efeito da polarização Galáctica.

Esses histogramas incluem todas as estrelas do catálogo. Desse modo, também pode ser que o suposto alinhamento tenha sido mascarado por outros padrões ou pela componente aleatória. Mais do que isso, o mapa da figura 3.14 ainda pode ajudar para a confirmação de que existe esse alinhamento. Aliás, também podemos começar a supor a existência de um campo em larga escala que seja alinhado com a Barra, o que foi pela primeira vez proposto por Magalhães et al. (2009). Assim, talvez o mapa da figura 3.13 represente melhor a configuração do campo para estruturas mais locais. Por outro lado, o mapa da 
figura 3.14 pode representar uma configuração mais global.
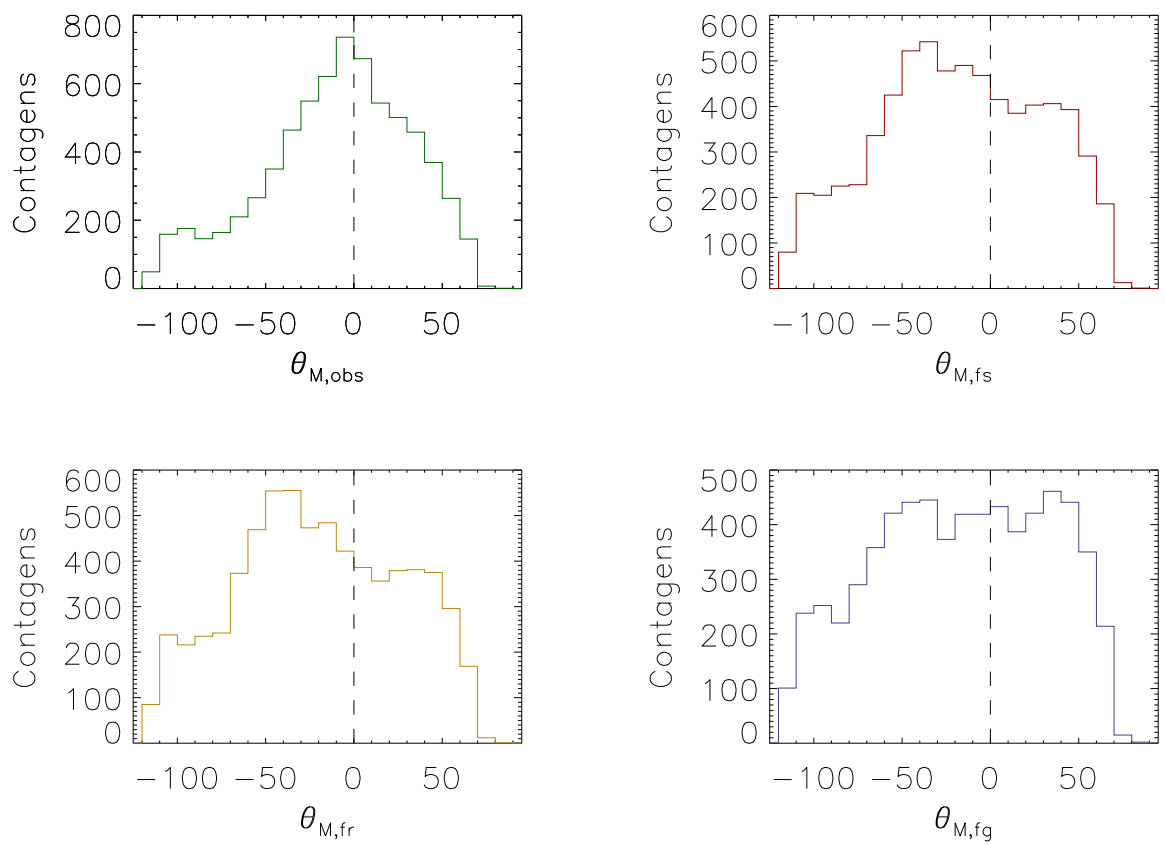

Figura 3.15: Histogramas para $\theta_{M}$, ângulo entre os vetores de polarização e a direção da GNM. O gráfico superior à esquerda apresenta os ângulos utilizando a polarização observada; superior à direita polarização corrigida por foreground de Schmidt (1976); inferior à esquerda polarização corrigida por foreground de Rodrigues et al. (1997); e inferior à direita polarização corrigida por foreground deste trabalho.

A origem de um campo coerente em larga escala para a PNM foi discutida por Mao et al. (2008). Eles chegaram à conclusão de que o melhor mecanismo para explicar a existência desse campo é um dínamo acionado por raios cósmicos (cosmic-ray driven dynamo). E incluindo modificações devido à força de maré, ele pode explicar que exista um campo alinhado com a Ponte pan-Magelânica. Contudo, para confirmar esta hipótese, em primeiro lugar é preciso que sejam feitas simulações MHD utilizando os parâmetros da PNM. Em segundo lugar, são necessárias mais medidas de rotação Faraday para entender melhor a configuração do campo na linha de visada. Por fim, para o campo no plano do céu, necessita-se de maior certeza de que a polarização de foreground esteja sendo subtraída corretamente para que os mapas de polarização de fato representem a geometria do campo no plano do céu. 


\subsection{Determinação da Intensidade do Campo Magnético}

Após conhecer a direção do campo magnético no plano do céu, o próximo passo foi determinar a sua intensidade. Para tanto, foi utilizado o método CF modificado por Falceta-Gonçalves et al. (2008), o qual já foi explicado na seção 1.3.

Para o emprego desse método, é necessário conhecer a dispersão dos vetores de polarização $(\delta \theta)$, dispersão de velocidade do gás na linha de visada $\left(\delta V_{L O S}\right)$ e densidade de massa do gás $(\rho)$. As dispersões dos vetores de polarização foram estimadas pelos ajustes das gaussianas nos histogramas de $\theta$. Para obter os outros dois elementos necessários para aplicação do método, foram utilizados mapas de dispersão de velocidade e densidade de coluna de HI (Stanimirović et al., 2004, 1999). A determinação dessas grandezas para cada campo está descrita a seguir.

\subsubsection{Dispersão de Velocidade do HI}

Para obter a dispersão de velocidade para cada campo, foi utilizado um mapa de $\delta V_{L O S}$ do HI, derivado através da análise do segundo-momento para imagens de HI combinadas, obtidas pelo ATCA e Parkes (Stanimirović et al., 2004). A figura 3.16 apresenta esse mapa.

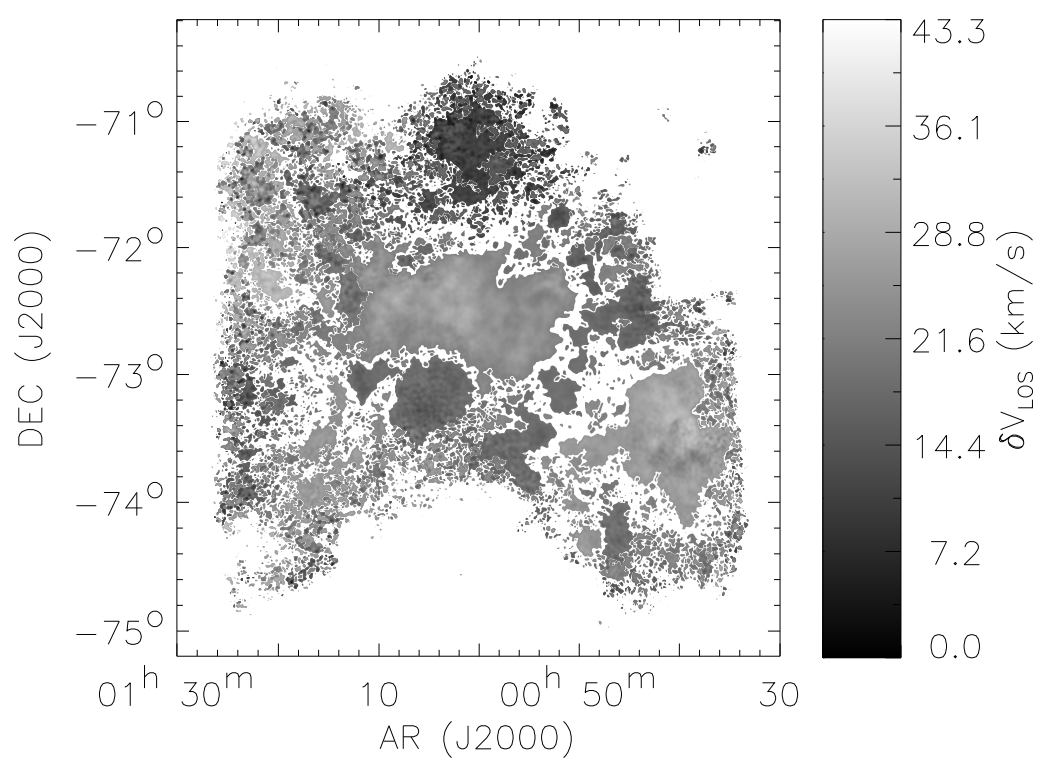

Figura 3.16: Mapa de dispersão de velocidade do HI na linha de visada.

Como o mapa compreende apenas o corpo da PNM, só foi possível a determinação local 
de $\delta V_{L O S}$ para os campos PNM01-19. Para os demais, campos na Ponte pan-Magelânica, foi admitido o valor médio para a Ponte, que é de $20 \mathrm{~km} / \mathrm{s}$ (Brüns et al., 2005).

A determinação local para os 19 campos pertencentes ao corpo da PNM foi feita utilizando o IDL. Recortamos imagens menores, com 8 x 8 minutos de arco, e com as mesmas coordenadas centrais dos campos CCD. Então, para cada imagem menor, calculava-se uma média das contagens dos pixeis e esse valor foi empregado como o $\delta V_{L O S}$ do campo. Estimamos a incerteza como sendo o desvio padrão da média das contagens desses pixeis.

\subsubsection{Densidade de Massa do HI}

Para obter a densidade de massa para cada campo, foi utilizado um mapa de densidade de coluna de HI, obtido pela combinação de imagens do ATCA e Parkes (Stanimirović et al., 1999), e integrando os perfis de HI em velocidade. A figura 3.17 apresenta o mapa.

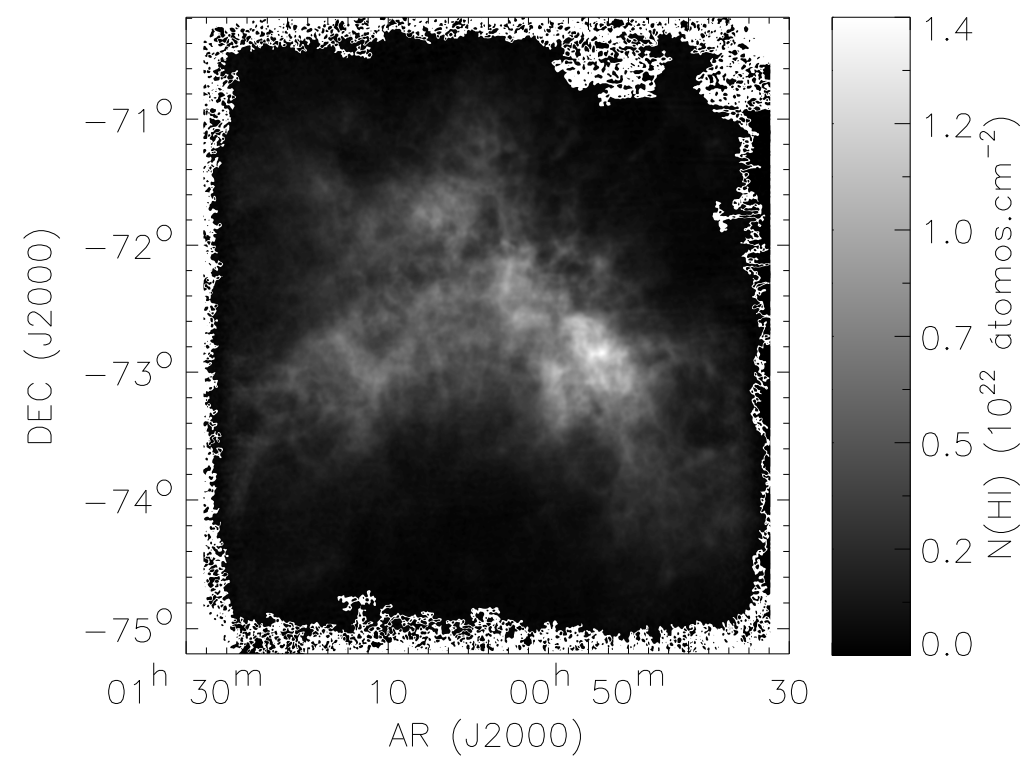

Figura 3.17: Mapa de densidade de coluna de HI.

Primeiramente, estimamos o $N(H I)$ local para cada campo. Mais uma vez, como o mapa compreende apenas o corpo da PNM, apenas os campos PNM01-19 puderam receber estimativas locais de $N(H I)$. Para os demais campos, localizados na Ponte, utilizamos o valor médio para a Ponte pan-Magelânica, que é de $5 \times 10^{20}$ átomos.cm $^{-2}$ (Brüns et al., 2005).

A determinação local foi feita do mesmo modo que no caso da obtenção de $\delta V_{L O S}$. A 
única diferença é que, antes de calcular as médias e os desvios padrões das médias, houve a necessidade de aplicar uma correção $(f)$, devido à autoabsorção do $\mathrm{H}$ ao longo da linha de visada (equação 3.14). Essa correção é necessária para densidades de coluna maiores do que $2,5 \times 10^{21}$ átomos.cm ${ }^{-2}$, como é explicado em Stanimirović et al. (1999). Ela foi aplicada pixel a pixel, antes da obtenção de $N(H I)$ local para cada campo.

$$
f= \begin{cases}1+0,667\left(\log N_{H I}-21,4\right) & ; \log N_{H I}>21,4 \\ 1 & ; \log N_{H I} \leq 21,4 .\end{cases}
$$

Em seguida, tivemos que assumir uma profundidade para a PNM, para que fosse possível converter densidade de coluna em densidade numérica, $n_{H}=N(H I) / d$. Subramanian e Subramaniam (2009) utilizaram a dispersão nas distribuições de magnitudes e cores de estrelas red clump para determinar a profundidade da PNM ao longo da linha de visada. Obtiveram para a Barra da PNM $d=(4,90 \pm 1,23)$ kpc e para o disco $d=(4,23 \pm 1,48) \mathrm{kpc}$. Então, utilizamos o valor da Barra para os campos pertencentes à região II de Schmidt (1976), e o valor do disco para os demais.

Por fim, a densidade de massa pôde ser obtida, $\rho=\gamma m_{H} n_{H}$. Assumimos $\gamma=1,22$, que é o peso molecular equivalente ao considerar as abundâncias da PNM (Mao et al., 2008). Determinamos as incertezas para $n_{H}$ e $\rho$ através da propagação de incertezas.

\subsubsection{Aplicação do Método CF}

Tendo em mãos todos os elementos, a intensidade para o campo magnético pôde ser calculada. Primeiramente, determinamos a intensidade do campo magnético aleatório, assumindo equipartição entre densidades de energias turbulentas magnética e cinética do gás (equação 1.18). Posteriormente, estimamos a intensidade do campo magnético no plano do céu, através da equação 1.19, que resume o método CF modificado por FalcetaGonçalves et al. (2008).

A tabela 3.3 mostra todos os valores locais para dispersão de velocidade do HI na linha de visada, densidade numérica de HI, intensidade do campo magnético turbulento e intensidade do campo magnético em larga escala projetado no plano do céu. Para os campos que apresentaram mais de um padrão de polarização, estimamos a intensidade de

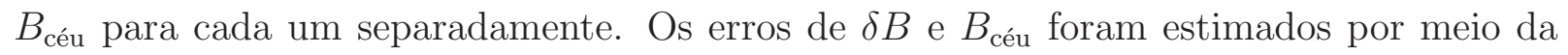


propagação de incertezas. As dispersões para os ângulos de polarização para cada campo podem ser vistas na tabela A.1, no apêndice A.

Tabela 3.3 - Parâmetros locais do MI dos nossos campos na PNM.

\begin{tabular}{|c|c|c|c|c|c|}
\hline PNM & $\delta V_{L O S}(\mathrm{~km} / \mathrm{s})$ & $n_{H}\left(\right.$ átomos.cm $\left.{ }^{-3}\right)$ & $\delta B(\mu \mathrm{G})$ & $B_{\text {céu }_{1}}(\mu \mathrm{G})$ & $B_{\text {céu }_{2}}(\mu \mathrm{G})$ \\
\hline 01 & $(12,643 \pm 0,078)$ & $(0,222 \pm 0,085)$ & $(3,47 \pm 0,44)$ & $(5,44 \pm 0,70)$ & $(4,75 \pm 0,77)$ \\
\hline 02 & $(14,763 \pm 0,091)$ & $(0,200 \pm 0,077)$ & $(3,85 \pm 0,48)$ & $(0,59 \pm 0,16)$ & $(7,0 \pm 1,6)$ \\
\hline 03 & $(24,228 \pm 0,075)$ & $(0,31 \pm 0,12)$ & $(7,9 \pm 1,0)$ & $(-0,29 \pm 0,17)$ & - \\
\hline 04 & $(25,723 \pm 0,072)$ & $(0,40 \pm 0,15)$ & $(9,5 \pm 1,2)$ & $(1,94 \pm 0,81)$ & $(40 \pm 12)$ \\
\hline 05 & $(13,799 \pm 0,093)$ & $(0,38 \pm 0,15)$ & $(4,95 \pm 0,62)$ & - & - \\
\hline 06 & $(19,690 \pm 0,085)$ & $(0,36 \pm 0,14)$ & $(6,86 \pm 0,86)$ & $(-4,62 \pm 0,72)$ & - \\
\hline 07 & $(27,824 \pm 0,045)$ & $(0,36 \pm 0,14)$ & $(9,7 \pm 1,2)$ & $(0,70 \pm 0,81)$ & $(10,9 \pm 1,5)$ \\
\hline 08 & $(22,09 \pm 0,13)$ & $(0,226 \pm 0,087)$ & $(6,13 \pm 0,77)$ & $(2,00 \pm 0,29)$ & $(9,2 \pm 1,4)$ \\
\hline 09 & $(26,332 \pm 0,066)$ & $(0,34 \pm 0,13)$ & $(8,9 \pm 1,1)$ & $(17,2 \pm 2,2)$ & - \\
\hline 10 & $(23,764 \pm 0,063)$ & $(0,34 \pm 0,13)$ & $(8,0 \pm 1,0)$ & $(8,1 \pm 1,1)$ & $(11,2 \pm 1,6)$ \\
\hline 11 & $(22,443 \pm 0,071)$ & $(0,35 \pm 0,13)$ & $(8,3 \pm 1,4)$ & $(18,6 \pm 3,3)$ & - \\
\hline 12 & $(22,200 \pm 0,049)$ & $(0,181 \pm 0,070)$ & $(5,9 \pm 1,0)$ & $(12,1 \pm 2,6)$ & - \\
\hline 13 & $(19,89 \pm 0,14)$ & $(0,35 \pm 0,14)$ & $(7,4 \pm 1,3)$ & $(9,2 \pm 2,3)$ & - \\
\hline 14 & $(21,08 \pm 0,14)$ & $(0,30 \pm 0,11)$ & $(7,2 \pm 1,3)$ & - & - \\
\hline 15 & $(23,31 \pm 0,11)$ & $(0,29 \pm 0,11)$ & $(7,9 \pm 1,4)$ & $(7,5 \pm 1,5)$ & - \\
\hline 16 & $(20,77 \pm 0,12)$ & $(0,121 \pm 0,046)$ & $(4,53 \pm 0,79)$ & $(4,9 \pm 2,1)$ & $(6,3 \pm 3,8)$ \\
\hline 17 & $(20,602 \pm 0,069)$ & $(0,212 \pm 0,081)$ & $(6,0 \pm 1,0)$ & $(4,4 \pm 1,5)$ & $(16,3 \pm 3,8)$ \\
\hline 18 & $(20,842 \pm 0,084)$ & $(0,129 \pm 0,050)$ & $(4,70 \pm 0,82)$ & $(-1,93 \pm 0,53)$ & - \\
\hline 19 & $(18,96 \pm 0,14)$ & $(0,099 \pm 0,038)$ & $(3,74 \pm 0,66)$ & - & - \\
\hline 20 & $\sim 20$ & $(0,0249 \pm 0,0096)$ & $(1,98 \pm 0,35)$ & $(2,97 \pm 0,94)$ & - \\
\hline 21 & $\sim 20$ & $(0,0249 \pm 0,0096)$ & $(1,98 \pm 0,35)$ & $(13,1 \pm 2,4)$ & - \\
\hline 22 & $\sim 20$ & $(0,0249 \pm 0,0096)$ & $(1,98 \pm 0,35)$ & $(0,83 \pm 0,58)$ & $(3,2 \pm 4,1)$ \\
\hline 23 & $\sim 20$ & $(0,0249 \pm 0,0096)$ & $(1,98 \pm 0,35)$ & $(2,53 \pm 0,78)$ & - \\
\hline 24 & $\sim 20$ & $(0,0249 \pm 0,0096)$ & $(1,98 \pm 0,35)$ & $(1,76 \pm 0,49)$ & - \\
\hline 25 & $\sim 20$ & $(0,0249 \pm 0,0096)$ & $(1,98 \pm 0,35)$ & - & - \\
\hline 26 & $\sim 20$ & $(0,0249 \pm 0,0096)$ & $(1,98 \pm 0,35)$ & $(1,28 \pm 0,47)$ & - \\
\hline 27 & $\sim 20$ & $(0,0249 \pm 0,0096)$ & $(1,98 \pm 0,35)$ & $(2,76 \pm 0,83)$ & $(12,5 \pm 3,1)$ \\
\hline 28 & $\sim 20$ & $(0,0249 \pm 0,0096)$ & $(1,98 \pm 0,35)$ & $(6,0 \pm 1,2)$ & - \\
\hline
\end{tabular}

Nota-se que três campos apresentaram intensidades negativas para o campo magnético no plano do céu - PNM 03, 06 e 18. Estes são também os campos onde a dispersão para os ângulos de polarização foram maiores, todas acima de $\pi / 4$. Para dispersões dessa ordem, o campo magnético pode conter apenas a componente turbulenta ou ter uma inclinação bastante alta com relação ao plano do céu. Como sabemos de medidas de rotação Faraday 
que o campo magnético na linha de visada é bastante baixo, $(0,19 \pm 0,06) \mu \mathrm{G}$ (Mao et al., 2008), é de se esperar que nessas regiões exista apenas campo turbulento.

Foi obtido um campo turbulento de $\delta B=(2,920 \pm 0,098) \mu \mathrm{G}$, a partir da média ponderada pelas incertezas para todos os campos. Para o campo em larga escala projetado no plano do céu, obteve-se $B_{\text {céu }}=(1,84 \pm 0,11) \mu \mathrm{G}$, também utilizando a média ponderada pelas incertezas e considerando as duas componentes observadas. Para o caso de campos que apresentaram mais de uma, os valores negativos foram desprezados para a média. Percebe-se que a intensidade do campo turbulento médio é maior do que a do campo em larga escala, quer dizer, na PNM o campo turbulento domina em relação ao de larga escala. Esse resultado está em acordo com o observado para outras galáxias irregulares (Chyży et al., 2011). Porém, no caso da PNM, a razão entre as componentes regular e turbulenta é um pouco maior.

A dispersão para os valores de $B_{\text {céu }}$ obtidos é bastante alta. Em muitos casos observa-se campos muito acima do valor médio calculado, chegando a cerca de vinte vezes maior. É bem provável que nesses casos o campo em larga escala esteja associado a estruturas locais. Na seção 4.1 estudaremos possíveis correlações entre os padrões de polarização/campo magnético e objetos relacionados ao MI observados nestes campo. 
Capítulo 4

\section{O Meio Interestelar da PNM}

Neste capítulo são apresentadas algumas correlações entre polarização e o MI da PNM padrões de polarização com estruturas do MI e polarização com avermelhamento. Também é feita uma avaliação da importância dinâmica que o campo magnético deve possuir para essa galáxia.

\subsection{Correlação entre os Padrões de Polarização e Estruturas do MI}

Um estudo interessante que pôde ser realizado foi tentar associar os padrões de polarização a estruturas observadas no MI da PNM. Para tanto, procuramos, dentro da base de dados Simbad, por objetos relacionados a ele (regiões HII, nuvens moleculares, shells, nebulosas planetárias etc.) nos nossos campos CCD.

Após obter a lista de objetos por campo, construímos os mapas de polarização para cada um. Utilizamos o catálogo com polarização corrigida pelo foreground obtido neste trabalho e as estrelas com $P / \sigma_{P}>3$. Os mapas foram compostos em cima de imagens do Spitzer/MIPS em $160 \mu \mathrm{m}$. Para facilitar a correlação visual entre os mapas e as estruturas, adicionamos neles a localização de cada uma.

Na figura 4.1 podem ser visualizados os mapas para dois campos interessantes - PNM 09 e 11. Estes são exemplos que apresentaram padrões de polarização bastante acentuados. O campo PNM09 contém dois shells e um objeto com linha de emissão, e o PNM11 quatro shells. Para eles, o campo magnético projetado no plano do céu apresentou alta intensidade, com erros bem determinados - menores que 20\%. Observações de polarimetria óptica já mostraram que algumas estruturas em larga escala no MI - as quais podem ter sido geradas pela interação de ventos estelares e explosões de supernovas com o MI - são magnetizadas, 
podendo possuir campos magnéticos de até algumas dezenas de $\mu \mathrm{G}$, por exemplo, IRAS Vela Shell (Pereyra e Magalhães, 2007) e NGC 2100 (Wisniewski et al., 2007). Assim, tais campos magnéticos relativamente altos, devem ser associados a esses shells.

Vale ressaltar que o campo PNM09 está localizado na Barra da PNM, e seu padrão de polarização possui ângulo alinhado com a Barra. Por outro lado, o campo PNM11 se encontra no início da Asa da PNM, e possui ângulo para o padrão de polarização apontando na direção da Ponte.
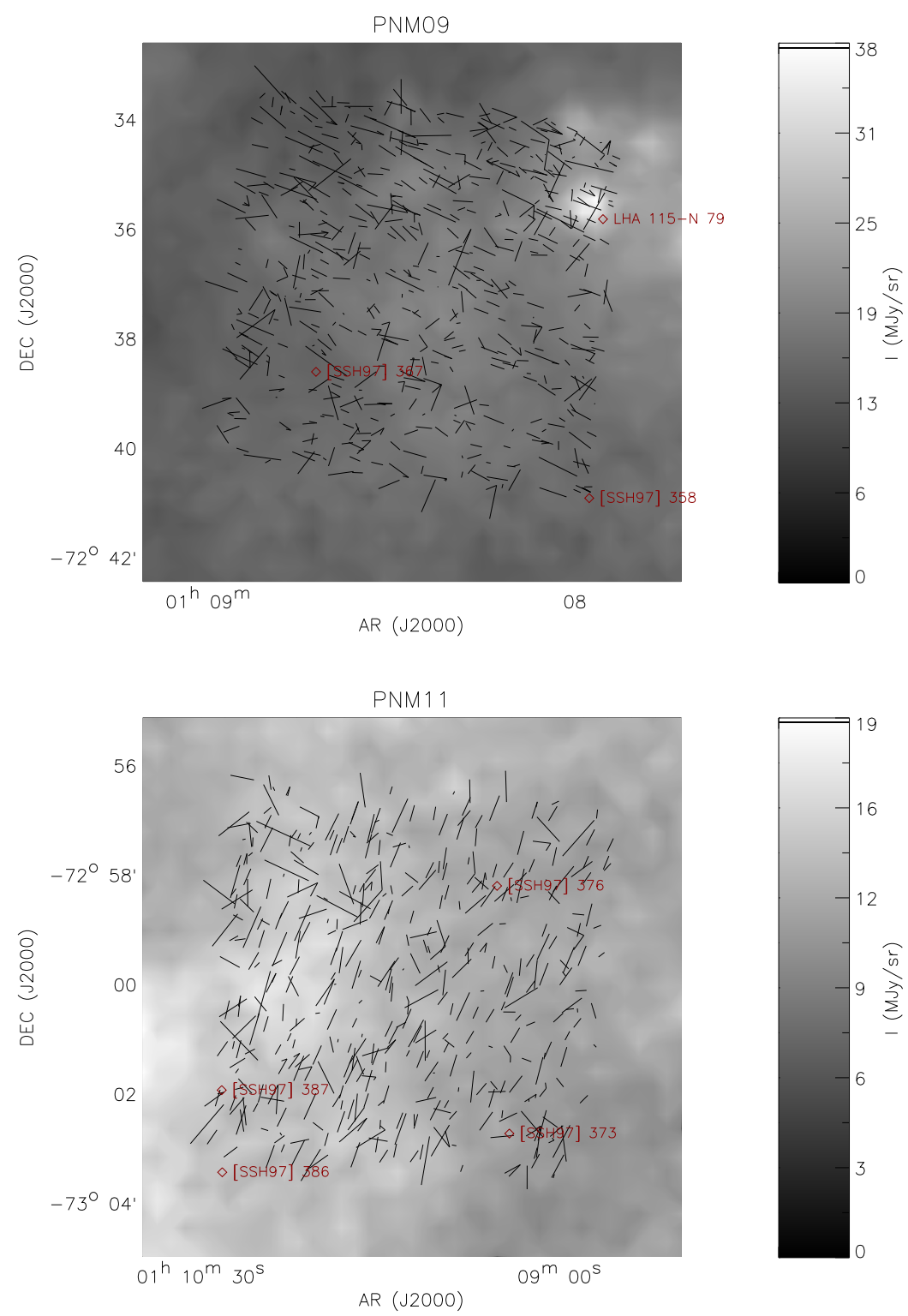

Figura 4.1: Mapas de polarização, para os campos PNM 09 e 11, sobrepostos a uma imagem do Spitzer/MIPS em $160 \mu \mathrm{m}$. Os diamantes vermelhos representam a localização da coordenada central de estruturas do MI, seguidos por seus nomes. 
Uma característica que se mostra evidente na maior parte dos mapas é que os vetores de polarização não possuem direções muito regulares, novamente revelando que o campo turbulento se sobressai em relação ao campo regular. Um caso interessante de se comentar é o campo PNM06. Ele foi aquele a possuir o maior número de objetos relacionados a MI. O seu mapa pode ser visualizado na figura 4.2. Ele contém um remanescente de supernova, um shell, dois objetos com linha de emissão, uma nuvem escura, uma nuvem molecular e três regiões HII. As regiões HII são conhecidas como berçário de estrelas, ou seja, deve haver formação estelar acontecendo nelas, e o feedback desse processo pode ajudar na destruição de campos regulares (Chyży et al., 2003). Acredita-se que as explosões de supernovas são uma das maiores fontes de injeção de turbulência no MI (McCray e Snow, 1979), o que também pode colaborar para a destruição de campos regulares. Assim, o fato de nessa região existir muita atividade acontecendo pode ter destruído algum campo regular preexistente, ou simplesmente estar impedindo ele de se formar.

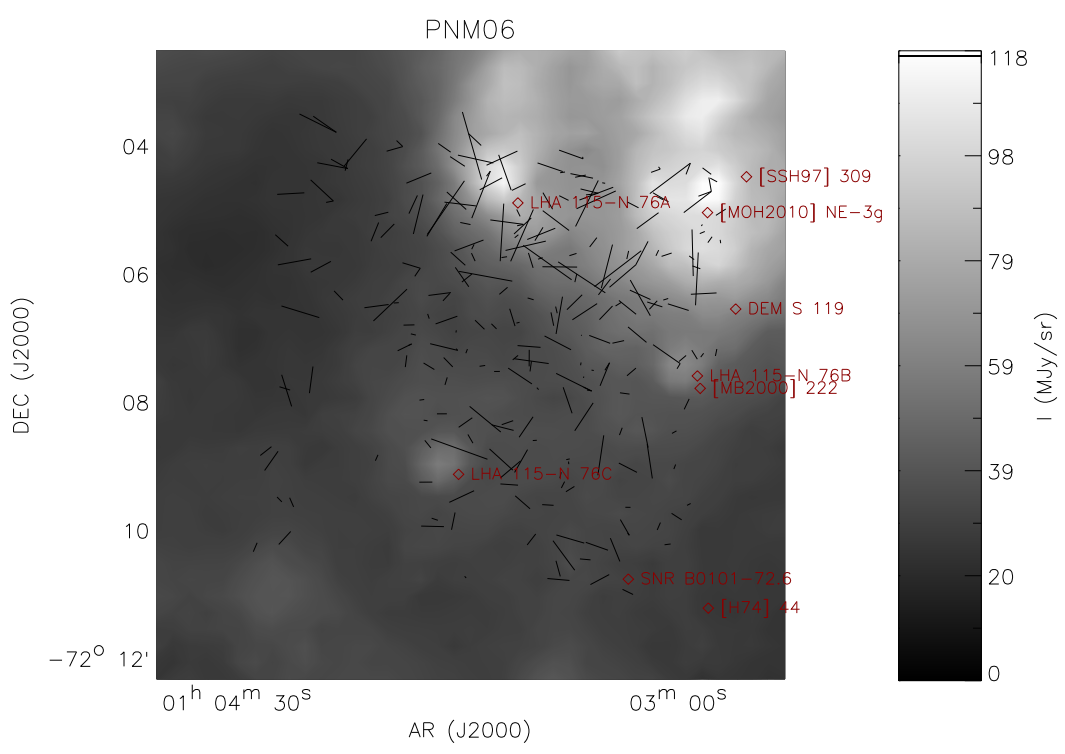

Figura 4.2: Mapa de polarização, para o campo PNM06, sobreposto a uma imagem do Spitzer/MIPS em $160 \mu \mathrm{m}$. Os diamantes vermelhos representam a localização da coordenada central de estruturas do MI, seguidos por seus nomes.

Os mapas para os outros campos estão apresentados no apêndice B, assim como a lista de objetos que se encontram em cada um (tabela B.1). Nesta seção foram discutidos apenas três campos por serem casos que se mostraram mais interessantes para comentar separadamente e servirem como exemplo para os demais. A tabela 4.1 mostra um resumo 
do que podemos supor para os padrões de polarização/campo magnético de cada campo.

Tabela 4.1 - Origem dos padrões de polarização/campo magnético de cada campo.

\begin{tabular}{|c|c|c|}
\hline PNM & \# de padrões & Suposta origem \\
\hline 01 & 2 & $B_{\text {céu }_{1}}$ associado ao shell e $B_{\text {céu }_{2}}$ associado ao MI geral \\
\hline 02 & 2 & $B_{\text {céu }}$ associado ao MI geral e $B_{\text {céu }}$ associado aos shells \\
\hline 03 & 1 & $B_{\text {céu }_{1}}$ associado ao shell, porém mascarado pela alta turbulência? \\
\hline 04 & 1 & $B_{\text {céu }_{1}}$ associado ao MI geral e $B_{\text {céu }_{2}}$ mal determinado \\
\hline 05 & 0 & Baixa estatística \\
\hline 06 & 1 & Campo regular sendo destruído pelo turbulento devido à alta atividade local \\
\hline 07 & 2 & $B_{\text {céu }}$ associado ao MI geral e $B_{\text {céu }}$ associado aos shells \\
\hline 08 & 2 & $B_{\text {céu }}$ associado ao MI geral e $B_{\text {céu }}$ associado aos shells \\
\hline 09 & 1 & $B_{\text {céu }}$ associado aos shells \\
\hline 10 & 2 & Aglomerado de estrelas presente, ambos padrões associados a ele? \\
\hline 11 & 1 & $B_{\text {céu }_{1}}$ associado aos shells \\
\hline 12 & 1 & $B_{\text {céu }_{1}}$ associado ao shell? \\
\hline 13 & 1 & $B_{\text {céu }_{1}}$ associado aos shells? \\
\hline 14 & 0 & Campo regular destruído pelo turbulento devido à alta atividade local \\
\hline 15 & 1 & $B_{\text {céu }_{1}}$ associado ao shell? \\
\hline 16 & 2 & $B_{\text {céu }}$ e $B_{\text {céu }}$ associados ao MI geral \\
\hline 17 & 2 & $B_{\text {céu }}$ associado ao MI geral e dúvida quanto a $B_{\text {céu }}$ \\
\hline 18 & 1 & Apenas campo turbulento? Região apresenta um pequeno excesso de poeira \\
\hline 19 & 0 & Baixa estatística \\
\hline 20 & 1 & $B_{\text {céu }_{1}}$ associado ao MI geral \\
\hline 21 & 1 & Padrão bastante acentuado! Porém, nada que explique, nem no óptico nem no IV \\
\hline 22 & 2 & $B_{\text {céu }_{1}}$ e $B_{\text {céu }_{2}}$ associados ao MI geral \\
\hline 23 & 1 & $B_{\text {céu }_{1}}$ associado ao MI geral \\
\hline 24 & 1 & $B_{\text {céu }_{1}}$ associado ao MI geral \\
\hline 25 & 0 & Baixa estatística \\
\hline 26 & 1 & $B_{\text {céu }_{1}}$ associado ao MI geral \\
\hline 27 & 2 & $B_{\text {céu }}$ associado ao MI geral e dúvida quanto a $B_{\text {céu }}$ \\
\hline 28 & 1 & Padrão acentuado, mas nada que justifique, nem no óptico nem no IV \\
\hline
\end{tabular}

\subsection{Relação entre Polarização e Avermelhamento}

Uma segunda correlação, possível de ser feita e de bastante interesse, é avaliar a relação entre polarização e avermelhamento. Ela é importante por oferecer uma indicação da eficiência da polarização na PNM.

Primeiramente, obtivemos o avermelhamento utilizando a relação entre densidade de 
coluna de HI, $N(H I)$, e avermelhamento, $A_{V}$, dada por Gordon et al. (2003). Para os campos que pertencem à região II de Schmidt (1976), utilizamos a relação para a Barra da PNM (equação 1.21). Para os demais, foi utilizada a relação para a Asa (equação 1.23). Determinamos as incertezas para $A_{V}$ a partir da propagação de incertezas.

Então, foi construído um gráfico, apresentado na figura 4.3, para avermelhamento em função da mediana da polarização de cada padrão. Para os campos com mais de um padrão de polarização, foram utilizadas as medianas de $P$ de cada padrão e o mesmo valor de $A_{V}$. Os campos sem padrão de polarização foram desconsiderados no gráfico. Por fim, foi ajustada uma reta para esses dados. Os valores obtidos para os coeficientes angular e linear, assim como suas incertezas, são apresentados no gráfico.

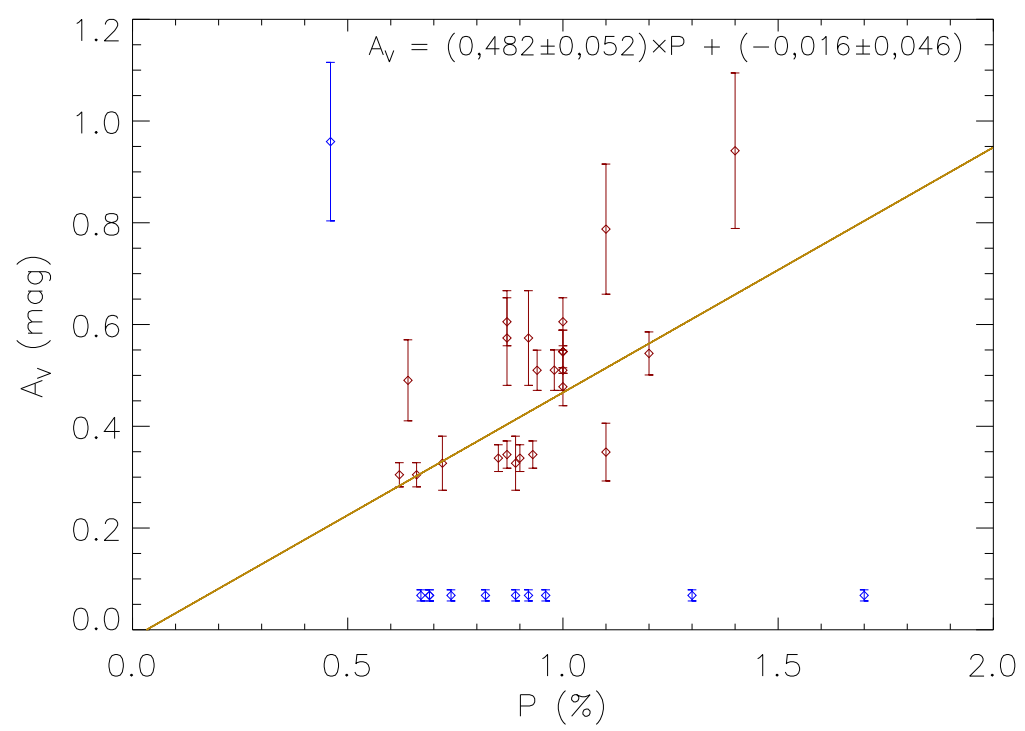

Figura 4.3: Avermelhamento em função da mediana da polarização de cada padrão. A reta em laranja foi obtida pelo ajuste de uma função linear para os dados. Os pontos em vermelho foram considerados para o ajuste, e os pontos em azul desconsiderados.

Os pontos apresentados em azul foram desconsiderados para o ajuste. Os da parte inferior do gráfico correspondem aos campos pertencentes à Ponte pan-Magelânica. Eles possuem o mesmo valor de $A_{V}$ devido ao fato de ter sido utilizado o mesmo valor médio de $N(H I)$ para determiná-los. Mais do que isso, foi utilizada a relação entre $N(H I)$ e $A_{V}$ da Asa, a qual pode também não ser apropriada. Somando esses dois problemas, nota-se que esses pontos estão bastante fora da tendência dos outros campos. Por isso, tomamos a decisão de desconsiderá-los para o ajuste. 
O ponto com menor valor para $P$, e na parte superior do gráfico, corresponde ao campo PNM13. Ele foi um dos campos onde as estrelas foram filtradas por magnitudes para melhor caracterização do padrão. Como o valor de $N(H I)$ utilizado para derivar $A_{V}$ foi obtido integrando em toda linha de visada, é bem possível que o valor de $A_{V}$ tenha sido superestimado para esse campo. Por isso ele ficou tão fora da tendência apresentada para os outros campos, mesmo levando em conta a sua incerteza alta. Assim, também decidimos excluí-lo do ajuste.

De modo geral, nota-se uma tendência bastante visível para os outros dados. Para avaliar a dependência entre $P$ e $A_{V}$, calculamos o coeficiente de Pearson para essas varáveis, o qual mede o quão correlacionadas estatisticamente essas grandezas estão. Obtivemos um coeficiente de 0,68, o que representa uma correlação razoavelmente alta.

Apesar de apresentarmos a relação com $P$ como variável independente, o mais natural é pensar que ela deva ser escrita com $A_{V}$. Fizemos o ajuste dessa forma apenas por questão de simplicidade, pois possuímos o erro para $A_{V}$ e não para mediana de $P$. Invertendo a relação obtemos que

$$
P=(2,08 \pm 0,12) \times A_{V}+(0,016 \pm 0,046)
$$

onde $P$ é dado em porcentagem; e $A_{V}$ em mag.

O coeficiente linear do ajuste é compatível com zero dentro de apenas um $1 \sigma$. Essa é outra indicação de que obtivemos um bom ajuste, pois não se espera observar nenhuma polarização para o caso de avermelhamento nulo, ou seja, ausência de poeira.

Essa relação nos mostra que para um avermelhamento de 1 mag temos uma polarização da ordem de 2\%. Para a Galáxia a relação típica é de $P / A_{V} \simeq 3$ (Serkowski et al., 1975), onde a polarização está sendo dada em porcentagem. Isso indica que na PNM a eficiência da polarização é mais baixa do que na VL. Podemos tentar justificar essa baixa eficiência por meio de dois argumentos:

(i) A alta turbulência que a PNM apresenta colabora para uma ineficiência para a polarização interestelar;

(ii) O campo magnético regular médio da PNM é cerca de 3 vezes menor do que o valor típico da Galáxia, sendo ele um agente importante para que a polarização aconteça, 
o fato dele ser mais fraco na PNM pode fazer com que a polarização seja menos eficiente.

\subsection{Importância Dinâmica do Campo Magnético}

Após determinar a geometria e a intensidade do campo magnético, uma coisa interessante a se fazer é avaliar a importância dinâmica que ele deve ter. Uma forma de estimá-la para cada componente de força é comparando suas densidades de energia (Beck, 2004). No caso, queremos estudar se a pressão magnética é tão importante quanto as forças provindas do movimento do gás. Desse modo, vamos obter as densidades de energia para quatro componentes.

- Movimento turbulento do gás:

$$
\epsilon_{\mathrm{tur}}=\frac{1}{2} \rho v_{\mathrm{tur}}^{2} \simeq \frac{3}{2} \rho \delta V_{\mathrm{LOS}}^{2}
$$

- Movimento de rotação do gás:

$$
\epsilon_{\mathrm{rot}}=\frac{1}{2} \rho v_{\mathrm{rot}}^{2}
$$

- Campo magnético total:

$$
\epsilon_{B_{\mathrm{tot}}}=\frac{B_{\mathrm{tot}}^{2}}{8 \pi} \simeq \frac{1}{8 \pi}\left(B_{\text {céu }}^{2}+B_{\mathrm{LOS}}^{2}+3 \delta B^{2}\right) .
$$

- Campo magnético regular:

$$
\epsilon_{B_{\mathrm{reg}}}=\frac{B_{\mathrm{reg}}^{2}}{8 \pi} \simeq \frac{1}{8 \pi}\left(B_{\mathrm{céu}}^{2}+B_{\mathrm{LOS}}^{2}\right) .
$$

Para a equação 4.2, assumimos que o movimento de turbulência é isotrópico, ou seja, podemos dizer que $v_{\text {tur }}^{2}=3 \delta V_{\mathrm{LOS}}^{2}$. Para a equação 4.4, assumimos que o campo turbulento é isotrópico, ou seja, $B_{\text {tur }}^{2}=3 \delta B^{2}$, e o campo regular pode ser representado pela soma dos campos no plano do céu e na linha de visada. Vamos calcular separadamente as densidades de energia para o campo magnético total e regular, afim de poder comparar a diferença entre ambas. A tabela 4.2 apresenta os valores admitidos para cada uma das grandezas envolvidas nas equações acima. 
Tabela 4.2 - Parâmetros médios para o gás e o campo magnético na PNM.

\begin{tabular}{cc}
\hline \hline Parâmetro & Valor \\
\hline$\rho=\gamma m_{H} n_{H}$ & $0,17 m_{H} \mathrm{~g} / \mathrm{cm}^{3}$ \\
$\delta V_{\mathrm{LOS}}$ & $21,50 \mathrm{~km} / \mathrm{s}$ \\
$v_{\text {rot }}$ & $60 \mathrm{~km} / \mathrm{s}$ \\
$B_{\text {céu }}$ & $1,84 \mu \mathrm{G}$ \\
$B_{\mathrm{LOS}}$ & $0,19 \mu \mathrm{G}$ \\
$\delta B$ & $2,92 \mu \mathrm{G}$ \\
\hline
\end{tabular}

A dispersão de velocidades na linha de visada foi obtida por meio do mesmo procedimento da seção 3.4.1. Calculamos o valor médio corresponde ao corpo da PNM, e não incluímos a Ponte. Para obter a densidade de massa do gás, empregamos o procedimento da seção 3.4.2. Calculamos $N(H I)$ médio para o corpo da PNM, e utilizamos a profundidade do disco da PNM para converter densidade de coluna em densidade numérica de H. A velocidade de rotação do gás utilizada foi a obtida por Stanimirović et al. (2004). Os campos magnéticos turbulento e no plano do céu considerados foram os obtidos neste trabalho, e o campo magnético na linha de visada, o determinado por Mao et al. (2008). A tabela 4.3 apresenta os valores obtidos para densidade de energia de cada componente.

Tabela 4.3 - Densidades de energia médias na PNM.

\begin{tabular}{cc}
\hline \hline Componente & Valor \\
\hline$\epsilon_{\text {tur }}$ & $1,96 \times 10^{-12} \mathrm{erg} / \mathrm{cm}^{3}$ \\
$\epsilon_{\text {rot }}$ & $5,10 \times 10^{-12} \mathrm{erg} / \mathrm{cm}^{3}$ \\
$\epsilon_{B_{\text {tot }}}$ & $1,15 \times 10^{-12} \mathrm{erg} / \mathrm{cm}^{3}$ \\
$\epsilon_{B_{\text {reg }}}$ & $0,14 \times 10^{-12} \mathrm{erg} / \mathrm{cm}^{3}$ \\
& \\
\hline
\end{tabular}

Nota-se que as densidades de energia de rotação e turbulência do gás e do campo magnético total possuem a mesma ordem de grandeza, indicando que a importância dinâmica de cada uma é aproximadamente igual. A densidade de energia do movimento de rotação do gás é a maior de todas, porém não o suficiente para que a força devido à rotação go- 
verne a dinâmica do gás da galáxia. A densidade de energia da componente turbulenta do gás é um pouco maior do que a do campo magnético total. Esse fato pode ser um pouco surpreendente, pois para obter o campo turbulento para cada um dos nossos campos, assumimos equipartição entre as densidades de energia de turbulência do campo magnético e cinética do gás. Porém, como não incluímos o gás da Ponte para obter o valor médio da segunda, isso pode ter levado a esse pequeno incremento para a densidade de energia. Mas, como estamos interessados apenas em conhecer as ordens de grandezas dessas densidades de energia, e não valores precisos, isso não se torna um problema. Por fim, a densidade de energia para a componente regular do campo magnético está uma ordem de grandeza abaixo das outras, ou seja, o campo turbulento que colabora para maior parte da pressão magnética.

Apesar desses cálculos terem sido efetuados de forma bastante aproximada, e de termos considerado apenas a componente do gás presente no corpo da PNM, eles foram importantes para destacar que o campo magnético tem de fato importância dinâmica para o movimento do gás, quer dizer, o estudo dele para essa galáxia possui importância vital para a compreensão da estrutura e da evolução do MI da PNM. 
Capítulo 5

\section{Conclusões e Perspectivas}

Este trabalho utilizou dados de polarimetria no óptico, obtidos no Cerro Tololo InterAmerican Observatory, com o intuito de estudar a estrutura do campo magnético na Pequena Nuvem de Magalhães. Esta é uma galáxia irregular satélite da Galáxia, e que possui um meio interestelar particularmente diferente do da Via Láctea. A redução dos dados polarimétricos gerou um catálogo com pouco mais de 7 mil estrelas, com polarizações em que os erros estão bem determinados. Por fim, o catálogo pôde ser utilizado para estudar o campo magnético nessa galáxia e para correlacionar a polarização com outras componentes e estruturas do MI da PNM. As principais conclusões deste trabalho estão resumidas a seguir.

- Observamos que uma eficiente subtração da polarização de foreground é essencial para determinar com precisão a geometria do campo magnético. Percebemos também que o famoso alinhamento entre o campo magnético e a Ponte pan-Magelânica pode ser um efeito dessa polarização Galáctica. Porém, a nossa análise não excluiu a possibilidade da sua existência, apenas mostrou esse alinhamento não ser tão intenso quanto o proposto por trabalhos anteriores.

- Nossos mapas de polarização demonstraram que o campo magnético no plano do céu possui uma configuração bastante irregular. Por outro lado, pode ser que existam dois padrões em larga escala, um alinhado com a Ponte e outro com a Barra da PNM. Mas, como o número de vetores que indicam essas estruturas não é muito grande, preferimos não afirmar com certeza a existência dessa configuração para o campo.

- A partir da dispersão dos vetores de polarização e da utilização do método CF, 
obtivemos um campo magnético regular de $B_{\text {céu }}=(1,84 \pm 0,11) \mu \mathrm{G}$ e um campo turbulento de $\delta B=(2,920 \pm 0,098) \mu \mathrm{G}$. Nota-se que o segundo é quase duas vezes maior do que o primeiro. Esse tipo de situação é observada para outras galáxias irregulares, indicando que nelas o campo turbulento domina com relação ao de larga escala. Essa conclusão também explica a grande irregularidade observada para os vetores de polarização. Não sabemos com exatidão a origem do campo magnético regular na PNM, e em outras galáxias irregulares. Porém, no caso da PNM, o mecanismo de um dínamo acionado por raios cósmicos parece eficiente para explicálo. Por outro lado, notamos que, para muitos dos nossos campos, pudemos associar o campo magnético regular a estruturas locais do MI.

- Conseguimos observar que muitos campos que apresentaram intensidades para o campo magnético projetado no plano do céu altas, eram dotados da presença de shells. O que pode indicar que esses altos valores observados estão associados a essas estruturas. Também foi possível notar alguns casos onde existia apenas campo turbulento. Para alguns, talvez ainda não houve tempo para que um campo em larga escala tenha sido gerado. Porém, em outros, ele pode ter existido e sido destruído por turbulência local.

- Analisamos a relação entre polarização e avermelhamento, e obtivemos $P / A_{V} \simeq 2$, onde a polarização é dada em porcentagem. Isso indica que na PNM a eficiência da polarização é mais baixa do que na Galáxia. Acreditamos que essa propriedade pode estar associada ao fato de que na PNM a turbulência é muito alta, o que dificulta o mecanismo de polarização interestelar. Outra opção é que a baixa eficiência esteja relacionada ao fato do campo regular ser mais baixo do que o da VL, ou ainda ser a soma desses dois efeitos.

- Determinamos, de modo um pouco simplificado, as densidades de energia do campo magnético total, campo magnético regular, movimento de rotação do gás e movimento de turbulência do gás. Chegamos à conclusão de que o campo magnético possui importância dinâmica da mesma ordem de grandeza das forças geradas pelo gás, sendo assim de fato importante o seu estudo. Também pudemos notar que a maior parte da pressão magnética é provinda da componente turbulenta do campo. 
Portanto, este estudo foi importante para uma melhor caracterização do campo magnético na Pequena Nuvem de Magalhães. Porém, ainda existe muito a ser feito.

Em primeiro lugar, ficou evidente que é necessário examinar com maior cuidado a polarização de foreground. Sem a convicção de que ela esteja sendo subtraída de forma correta, fica difícil determinar com precisão a geometria para o campo no plano do céu.

Um segundo problema que influenciou nossa análise foi não termos estimativas locais para os parâmetros do gás na Ponte. Desse modo, é necessário buscar por essas informações para refinar nossos resultados.

Por último, precisamos destacar que esse estudo cobriu apenas as regiões Nordeste, da Asa e início da Ponte pan-Magelânica. Assim sendo, é necessário um mapeamento do resto da PNM para que seja possível um conhecimento do quadro completo da galáxia. Mas o projeto SOUTH POL irá tomar dados polarimétricos no óptico, e mapear a polarização de todo o céu do hemisfério Sul. Com isso, será possível conhecer o quadro completo, não apenas para a PNM, como também para a GNM e para a Ponte, o que levará a uma compreensão maior de todo esse sistema. 


\section{Referências Bibliográficas}

Alfvén H., Existence of Electromagnetic-Hydrodynamic Waves, Nature, 1942, vol. 150, p. 405

Ardeberg A., Photoelectric photometry of stars in the Small Magellanic Cloud, A\&AS, 1980, vol. 42, p. 1

Ardeberg A., Maurice E., Observations of supergiant stars in the Small Magellanic Cloud, A\&AS, 1977, vol. 30, p. 261

Bastien P., Drissen L., Menard F., Moffat A. F. J., Robert C., St-Louis N., The variability of polarized standard stars, AJ, 1988, vol. 95, p. 900

Beck R., The Role of Magnetic Fields in Spiral Galaxies, Ap\&SS, 2004, vol. 289, p. 293

Bekki K., Models for the dynamical evolution of the Magellanic System. In The Magellanic System: Stars, Gas, and Galaxies, vol. 256 do IAU Symposium, 2009, pp 105-116

Bekki K., Chiba M., Formation and evolution of the Magellanic Clouds - I. Origin of structural, kinematic and chemical properties of the Large Magellanic Cloud, MNRAS, 2005, vol. 356 , p. 680

Bernard J.-P., Reach W. T., Paradis D., et al ., Spitzer Survey of the Large Magellanic Cloud, Surveying the Agents of a Galaxy's Evolution (sage). IV. Dust Properties in the Interstellar Medium, AJ, 2008, vol. 136, p. 919

Bessell M. S., Concerning the reddening of the Magellanic Clouds, A\&A, 1991, vol. 242, p. L17 
Bot C., Boulanger F., Lagache G., Cambrésy L., Egret D., Multi-wavelength analysis of the dust emission in the Small Magellanic Cloud, A\&A, 2004, vol. 423, p. 567

Bot C., Boulanger F., Rubio M., Rantakyro F., Millimeter dust continuum emission revealing the true mass of giant molecular clouds in the Small Magellanic Cloud, A\&A, 2007, vol. 471, p. 103

Bot C., Ysard N., Paradis D., Bernard J. P., Lagache G., Israel F. P., Wall W. F., Submillimeter to centimeter excess emission from the Magellanic Clouds. II. On the nature of the excess, A\&A, 2010, vol. 523, p. A20

Brüns C., Kerp J., Staveley-Smith L., Mebold U., Putman M. E., Haynes R. F., Kalberla P. M. W., Muller E., Filipovic M. D., The Parkes H I Survey of the Magellanic System, A\&A, 2005, vol. 432, p. 45

Cardelli J. A., Clayton G. C., Mathis J. S., The relationship between infrared, optical, and ultraviolet extinction, ApJ, 1989, vol. 345, p. 245

Chandrasekhar S., Fermi E., Magnetic Fields in Spiral Arms, ApJ, 1953, vol. 118, p. 113

Chyży K. T., Knapik J., Bomans D. J., Klein U., Beck R., Soida M., Urbanik M., Magnetic fields and ionized gas in the local group irregular galaxies IC 10 and NGC 6822, A\&A, 2003, vol. 405, p. 513

Chyży K. T., Weżgowiec M., Beck R., Bomans D. J., Magnetic fields in Local Group dwarf irregulars, A\&A, 2011, vol. 529, p. A94

Cioni M.-R. L., van der Marel R. P., Loup C., Habing H. J., The tip of the red giant branch and distance of the Magellanic Clouds: results from the DENIS survey, A\&A, 2000, vol. 359, p. 601

Davies R. D., Elliott K. H., Meaburn J., The nebular complexes of the large and small Magellanic Clouds, MmRAS, 1976, vol. 81, p. 89

Davis Jr. L., Greenstein J. L., The Polarization of Starlight by Aligned Dust Grains, ApJ, 1951, vol. 114, p. 206 
Demers S., Irwin M. J., Catalogue of blue stars between the Magellanic Clouds, A\&AS, 1991, vol. 91, p. 171

Falceta-Gonçalves D., Lazarian A., Kowal G., Studies of Regular and Random Magnetic Fields in the ISM: Statistics of Polarization Vectors and the Chandrasekhar-Fermi Technique, ApJ, 2008, vol. 679, p. 537

Fermi E., On the Origin of the Cosmic Radiation, Physical Review, 1949, vol. 75, p. 1169

Fitzpatrick E. L., Massa D., An analysis of the shapes of ultraviolet extinction curves. III - an atlas of ultraviolet extinction curves, ApJS, 1990, vol. 72, p. 163

Gaustad J. E., McCullough P. R., Rosing W., Van Buren D., A Robotic Wide-Angle H $\alpha$ Survey of the Southern Sky, PASP, 2001, vol. 113, p. 1326

Gordon K. D., Clayton G. C., Misselt K. A., Landolt A. U., Wolff M. J., A Quantitative Comparison of the Small Magellanic Cloud, Large Magellanic Cloud, and Milky Way Ultraviolet to Near-Infrared Extinction Curves, ApJ, 2003, vol. 594, p. 279

Hall J. S., Observations of the Polarized Light from Stars, Science, 1949, vol. 109, p. 166

Haynes R. F., Harnett S. W. J. I., Klein U., Wielebinski R., Buczilowski U. R., Bajaja E., Malin D., Murray J. D., Hunt A. J., Wark R., Sciacca L., Polarization in the Magellanic Clouds. In Galactic and Intergalactic Magnetic Fields, vol. 140 do IAU Symposium, 1990, p. 205

Hecht E., Optics. 4th edition, San Francisco: Addison Wesley, 2001

Heiles C., 9286 Stars: An Agglomeration of Stellar Polarization Catalogs, AJ, 2000, vol. 119 , p. 923

Heiles C. E., The Fifth ISM Phase as Revealed by Faraday Rotation. In American Astronomical Society Meeting Abstracts \#217, vol. 43 do Bulletin of the American Astronomical Society, 2011, p. 132.08

Heitsch F., Zweibel E. G., Mac Low M.-M., Li P., Norman M. L., Magnetic Field Diagnostics Based on Far-Infrared Polarimetry: Tests Using Numerical Simulations, ApJ, 2001, vol. 561, p. 800 
Henize K. G., Catalogues of H $\alpha$-EMISSION Stars and Nebulae in the Magellanic Clouds, ApJS, 1956, vol. 2, p. 315

Hiltner W. A., On the Presence of Polarization in the Continuous Radiation of Stars. II., ApJ, 1949, vol. 109, p. 471

Hindman J. V., A high resolution study of the distribution and motions of neutral hydrogen in the Small Cloud of Magellan, Australian Journal of Physics, 1967, vol. 20, p. 147

Hindman J. V., Kerr F. J., McGee R. X., A Low Resolution Hydrogen-line Survey of the Magellanic System. II. Interpretation of Results, Australian Journal of Physics, 1963, vol. 16 , p. 570

Hoang T., Lazarian A., Grain Alignment Induced by Radiative Torques: Effects of Internal Relaxation of Energy and Complex Radiation Field, ApJ, 2009, vol. 697, p. 1316

Howell S. B., CCD growth curves - Application to faint and crowded point sources. In CCDs in astronomy, vol. 8 do Astronomical Society of the Pacific Conference Series, 1990, pp 312-318

Howell S. B., Introduction to Differential Time-Series Astronomical Photometry Using Charged-Coupled Devices. In Astronomical CCD Observing and Reduction Techniques, vol. 23 do Astronomical Society of the Pacific Conference Series, 1992, p. 105

HPOL ., HPOL Synthetic UBVRI Filter Data for BD+25_727, Material Online, http://www.sal.wisc.edu/HPOL/tgts/BD+25_727.html, Acessado em 2011

Hsu J.-C., Breger M., On standard polarized stars, ApJ, 1982, vol. 262, p. 732

Irwin M. J., Kunkel W. E., Demers S., A blue stellar population in the H I bridge between the two Magellanic Clouds, Nature, 1985, vol. 318, p. 160

Israel F. P., Wall W. F., Raban D., Reach W. T., Bot C., Oonk J. B. R., Ysard N., Bernard J. P., Submillimeter to centimeter excess emission from the Magellanic Clouds. I. Global spectral energy distribution, A\&A, 2010, vol. 519, p. A67

Isserstedt J., Photoelectric photometry in the Small Magellanic Cloud, A\&AS, 1978, vol. 33, p. 193 
Jackson J. D., Classical Electrodynamics. 3rd edition, Hoboken: John Wiley \& Sons, Inc., 1998

Karttunen H., Kroeger P., Oja H., Poutanen M., Donner K. J., Fundamental Astronomy. 5th edition, Berlin: Springer, 2007

Kurt C. M., Dufour R. J., The Chemical Composition of H II Regions in the Magellanic Clouds: New Calculations Using Modern Atomic Data. In The Sixth Texas-Mexico Conference on Astrophysics: Astrophysical Plasmas - Near and Far, vol. 7 da Revista Mexicana de Astronomia y Astrofisica Conference Series, 1998, p. 202

Lasker B., Lattanzi M. G., McLean B. J., et al ., The Guide Star Catalog, Version 2.3.2 (GSC2.3) (STScI, 2006), VizieR Online Data Catalog, 2007, vol. 1305, p. 0

Lazarian A., Hoang T., Alignment of Dust with Magnetic Inclusions: Radiative Torques and Superparamagnetic Barnett and Nuclear Relaxation, ApJL, 2008, vol. 676, p. L25

Loiseau N., Klein U., Greybe A., Wielebinski R., Haynes R. F., Thermal and nonthermal radio emission from the Small Magellanic Cloud, A\&A, 1987, vol. 178, p. 62

Maciel W. J., Astrofísica do Meio Interestelar. Primeira edição, São Paulo: EDUSP, 2002

Magalhães A. M., Notas de Aula de Processos Radiativos - 4. Polarização, Material Online, ftp://astroweb.iag.usp.br/pub/mario/Talks/aga5735/4_Polarizacao.pdf, Acessado em 2012

Magalhães A. M., Gomes A. L., Vidotto A. A., Rodrigues C. V., Pereyra A., Wisniewski J., Bjorkman K., Bjorkman J., Meade M., Babler B. L., The magnetic field structure of the Small Magellanic Cloud. In The Magellanic System: Stars, Gas, and Galaxies, vol. 256 do IAU Symposium, 2009, pp 178-183

Magalhães A. M., Loiseau N., Rodrigues C. V., Piirola V., Magnetic field structure in the Small Magellanic Cloud. In Galactic and Intergalactic Magnetic Fields, vol. 140 do IAU Symposium, 1990, pp 255-+

Magalhães A. M., Rodrigues C. V., Margoniner V. E., Pereyra A., Heathcote S., High Precision CCD Imaging Polarimetry. In Polarimetry of the Interstellar Medium, vol. 97 do Astronomical Society of the Pacific Conference Series, 1996, p. 118 
Mao S. A., Gaensler B. M., Stanimirović S., Haverkorn M., McClure-Griffiths N. M., Staveley-Smith L., Dickey J. M., A Radio and Optical Polarization Study of the Magnetic Field in the Small Magellanic Cloud, ApJ, 2008, vol. 688, p. 1029

Massey P., UBVR CCD survey of the Magellanic clouds (Massey+, 2002), VizieR Online Data Catalog, 2002, vol. 2236, p. 0

Mathewson D. S., Ford V. L., Polarization measurements of stars in the Magellanic Clouds, AJ, 1970a, vol. 75, p. 778

Mathewson D. S., Ford V. L., The Magnetic-Field Structure of the Magellanic Clouds, ApJL, 1970b, vol. 160, p. L43+

McCray R., Snow Jr. T. P., The violent interstellar medium, ARA\&A, 1979, vol. 17, p. 213

Meixner M., Galliano F., Hony S., et al ., HERschel Inventory of The Agents of Galaxy Evolution (HERITAGE): The Large Magellanic Cloud dust, A\&A, 2010, vol. 518, p. L71

Mizuno N., Rubio M., Mizuno A., Yamaguchi R., Onishi T., Fukui Y., First Results of a CO Survey of the Small Magellanic Cloud with NANTEN, PASJ, 2001, vol. 53, p. L45

Muller E., Stanimirović S., Rosolowsky E., Staveley-Smith L., A Statistical Investigation of H I in the Magellanic Bridge, ApJ, 2004, vol. 616, p. 845

Pereyra A., Poeira e Campo Magnético em Regiões Densas do Meio Interestelar, São Paulo: Universidade de São Paulo, 2000, Tese de Doutorado

Pereyra A., Magalhães A. M., Polarimetry toward the IRAS Vela Shell. II. Extinction and Magnetic Fields, ApJ, 2007, vol. 662, p. 1014

Perie J., Rousseau M., Prevot L., Peyrin Y., Robin A., Positional reference stars in the Magellanic Clouds, A\&AS, 1991, vol. 90, p. 1

Planck Collaboration ., Planck early results. XVII. Origin of the submillimetre excess dust emission in the Magellanic Clouds, A\&A, 2011, vol. 536, p. A17 
Pradhan A. C., Murthy J., Pathak A., Observations of Far-ultraviolet Diffuse Emission from the Small Magellanic Cloud, ApJ, 2011, vol. 743, p. 80

Rodrigues C. V., Magalhães A. M., Coyne G. V., Piirola V., Dust in the Small Magellanic Cloud: Interstellar Polarization and Extinction, ApJ, 1997, vol. 485, p. 618

Rubio M., Garay G., Montani J., Thaddeus P., A (C-12)O survey of the Small Magellanic Cloud, ApJ, 1991, vol. 368, p. 173

Ruzmaikin A. A., Sokolov D. D., Shukurov A. M., Magnetic fields of galaxies. 1st edition, Berlin: Springer, 1988

Sanduleak N., Known and suspected late-type supergiants in the Small Magellanic Cloud, AJ, 1989, vol. 98, p. 825

Schmidt T., Polarization measurements and magnetic field structure within the Magellanic Clouds, A\&A, 1970, vol. 6, p. 294

Schmidt T., Starlight polarization in the Magellanic Cloud regions, A\&AS, 1976, vol. 24, p. 357

Seaton M. J., Interstellar extinction in the UV, MNRAS, 1979, vol. 187, p. 73P

Serkowski K., Methods of Experimental Physics, Vol. 12: Astrophysics, Part A: Optical and Infrared. 1st edition, New York: Academic Press, 1974

Serkowski K., Mathewson D. S., Ford V. L., Wavelength dependence of interstellar polarization and ratio of total to selective extinction, ApJ, 1975, vol. 196, p. 261

Sofue Y., CO observations of edge-on galaxies. 5. NGC 5907: Central deficiency of gas in an SC galaxy-merger in the bulge?, PASJ, 1994, vol. 46, p. 173

Stanimirović S., Staveley-Smith L., Dickey J. M., Sault R. J., Snowden S. L., The largescale HI structure of the Small Magellanic Cloud, MNRAS, 1999, vol. 302, p. 417

Stanimirović S., Staveley-Smith L., Jones P. A., A New Look at the Kinematics of Neutral Hydrogen in the Small Magellanic Cloud, ApJ, 2004, vol. 604, p. 176 
Stanimirović S., Staveley-Smith L., van der Hulst J. M., Bontekoe T. R., Kester D. J. M., Jones P. A., Cool dust and gas in the Small Magellanic Cloud, MNRAS, 2000, vol. 315, p. 791

Subramanian S., Subramaniam A., Depth estimation of the Large and Small Magellanic Clouds, A\&A, 2009, vol. 496, p. 399

Tapia S., Preprints of the Steward Observatory. No. 831, 1988

Vangioni-Flam E., Lequeux J., Maucherat-Joubert M., Rocca-Volmerange B., Ultraviolet studies of the Magellanic Clouds. II - Internal extinction, formation of massive stars, comparison with other galaxies, A\&A, 1980, vol. 90, p. 73

Westerlund B. E., Review: an Overview of the Structure and Kinematics of the Magellanic Clouds. In The Magellanic Clouds, vol. 148 do IAU Symposium, 1991, pp 15-+

Wisniewski J. P., Bjorkman K. S., Magalhães A. M., Pereyra A., The Magnetic Field Structure of the LMC 2 Supershell: NGC 2100, ApJ, 2007, vol. 664, p. 296

Zweibel E. G., Magnetic field-line tangling and polarization measurements in clumpy molecular gas, ApJ, 1990, vol. 362, p. 545

Zweibel E. G., Heiles C., Magnetic fields in galaxies and beyond, Nature, 1997, vol. 385, p. 131 
Apêndice 



\section{Apêndice A}

\section{Histogramas para Ângulo e Intensidade da Polarização}

Neste apêndice, estão apresentados os histogramas para os ângulos de polarização e para sua intensidade, onde o segundo foi construído considerando apenas as estrelas que possuíam ângulo dentro de $3 \sigma$ do valor médio de $\theta$. Também é apresentada uma tabela com os parâmetros obtidos pelos ajustes.

\section{A.1 Campos com Um Padrão}
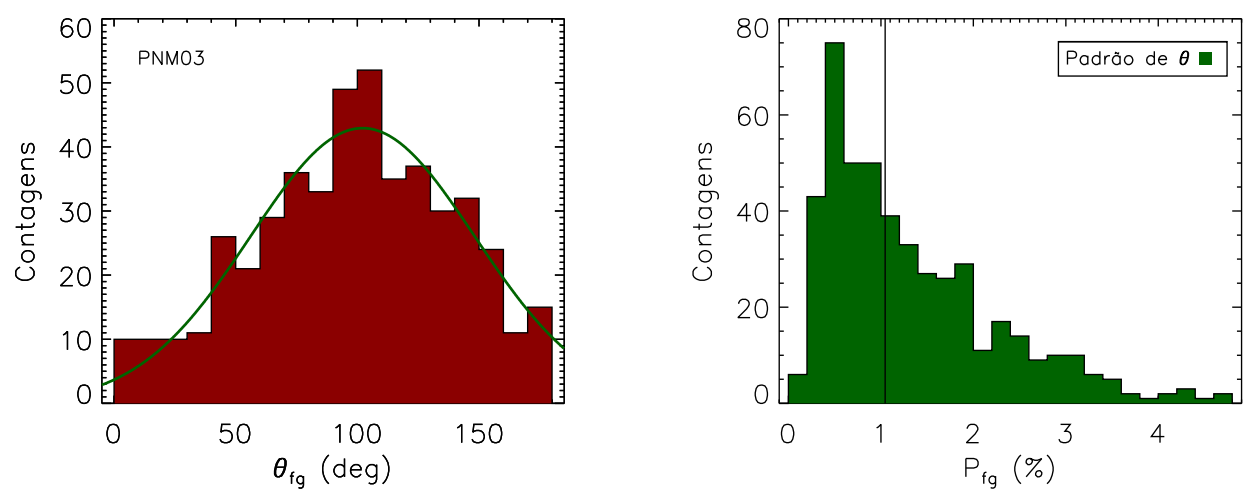

Figura A.1: Campos com apenas um padrão para $\theta$. Histogramas para o ângulo de polarização e a sua intensidade. A linha vertical no histograma de $P$ marca a sua mediana. 

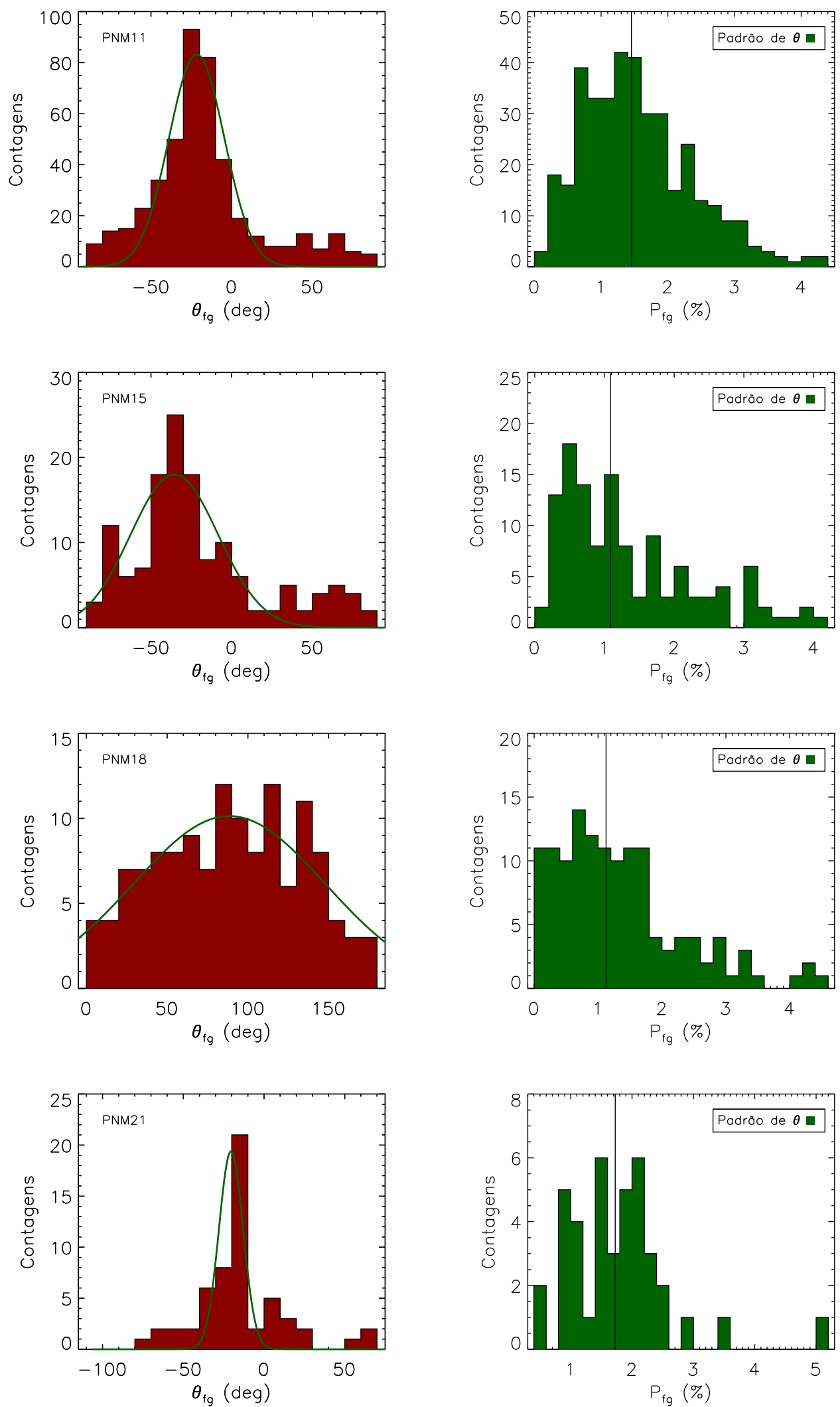

Figura A.2: Campos com apenas um padrão para $\theta$. Histogramas para o ângulo de polarização e a sua intensidade. A linha vertical no histograma de $P$ marca a sua mediana (continuação). 


\section{A.2 Campos com Dois Padrões}
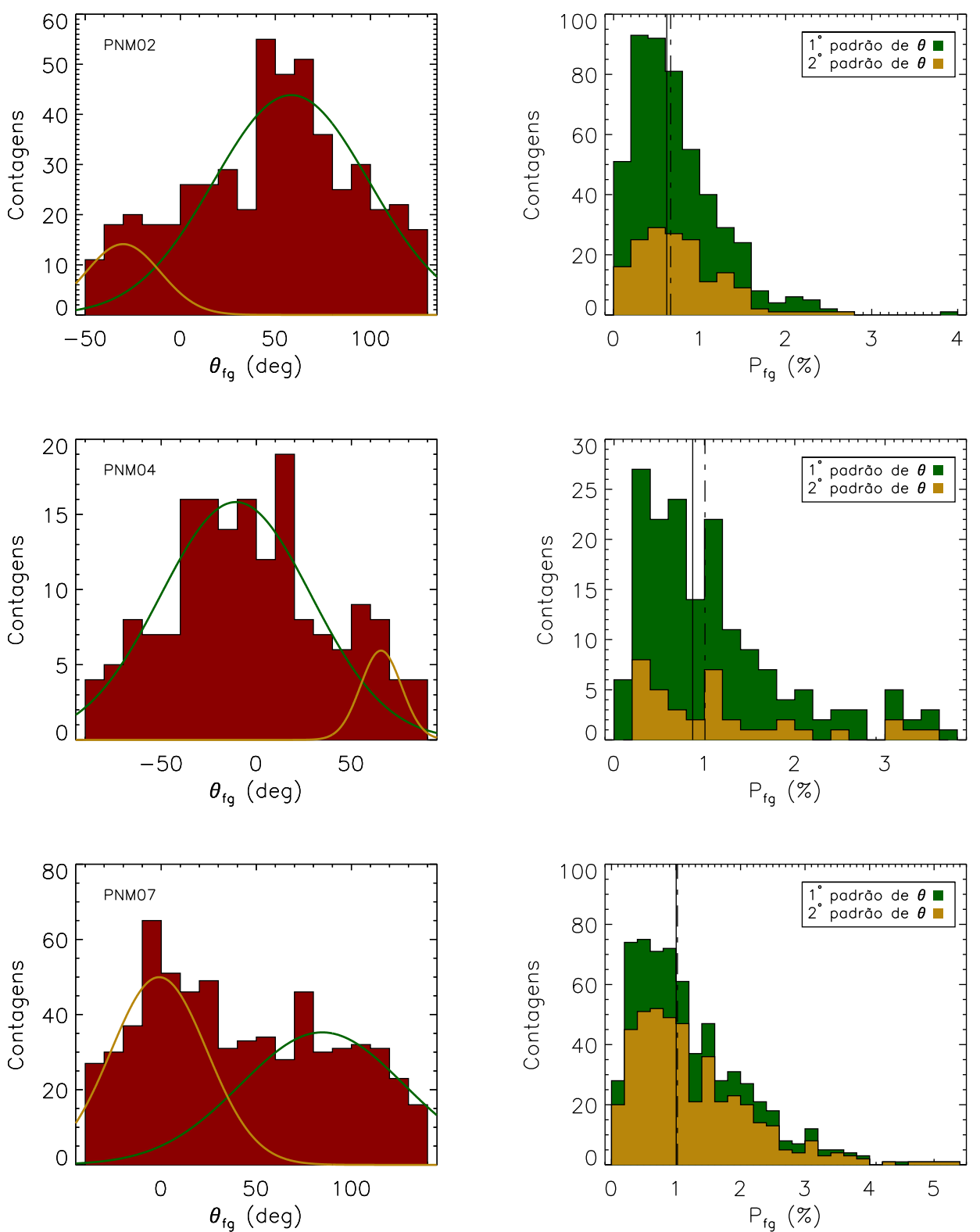

Figura A.3: Campos com dois padrões para $\theta$. Histogramas para o ângulo de polarização e a sua intensidade. As linhas verticais no histograma de $P$ marcam as medianas para o primeiro (linha sólida) e segundo padrão (linha sólida-pontilhada). 

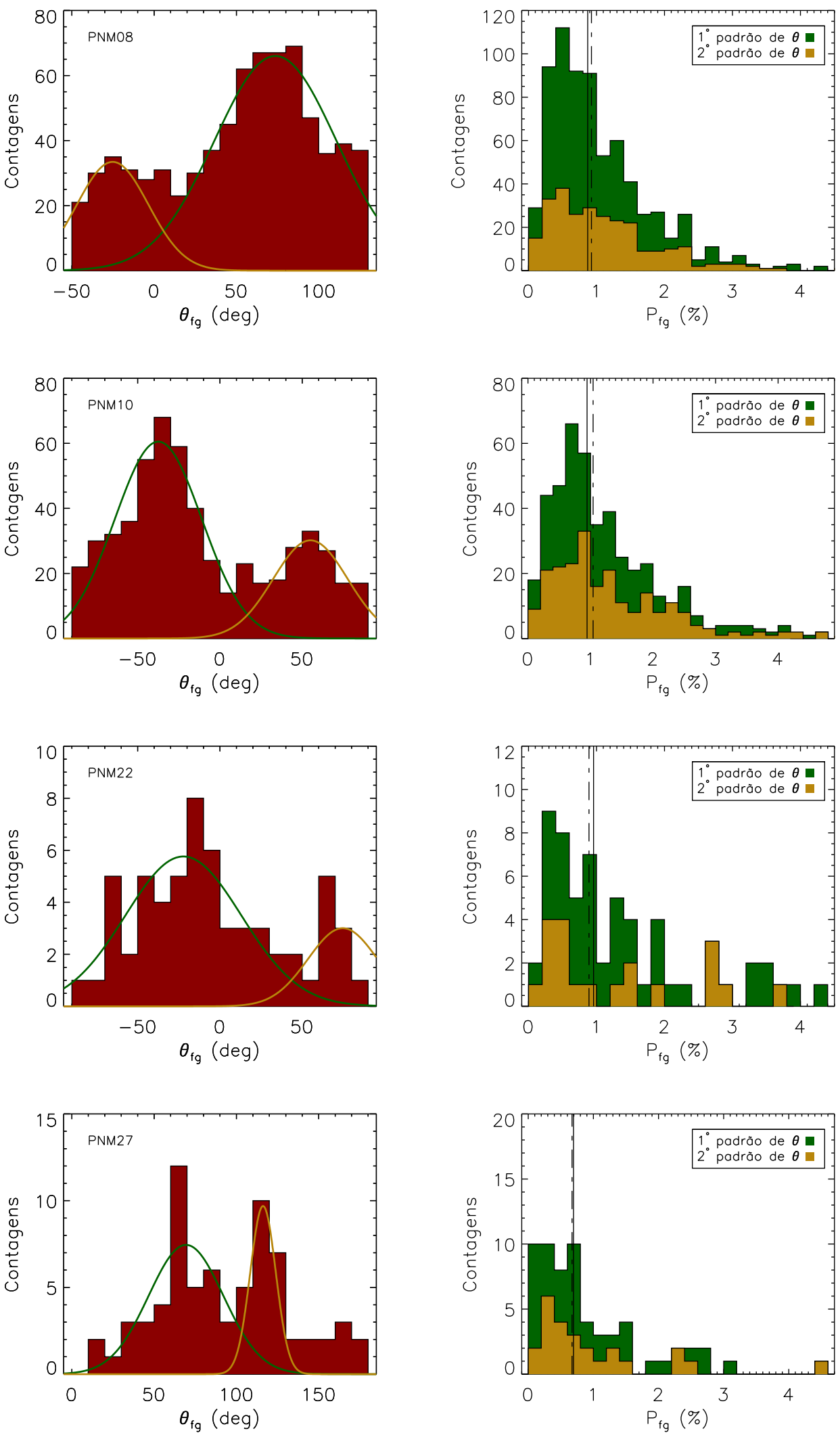

Figura A.4: Campos com dois padrões para $\theta$. Histogramas para o ângulo de polarização e a sua intensidade. As linhas verticais no histograma de $P$ marcam as medianas para o primeiro (linha sólida) e segundo padrão (linha sólida-pontilhada) (continuação). 


\section{A.3 Campos com Nenhum Padrão}
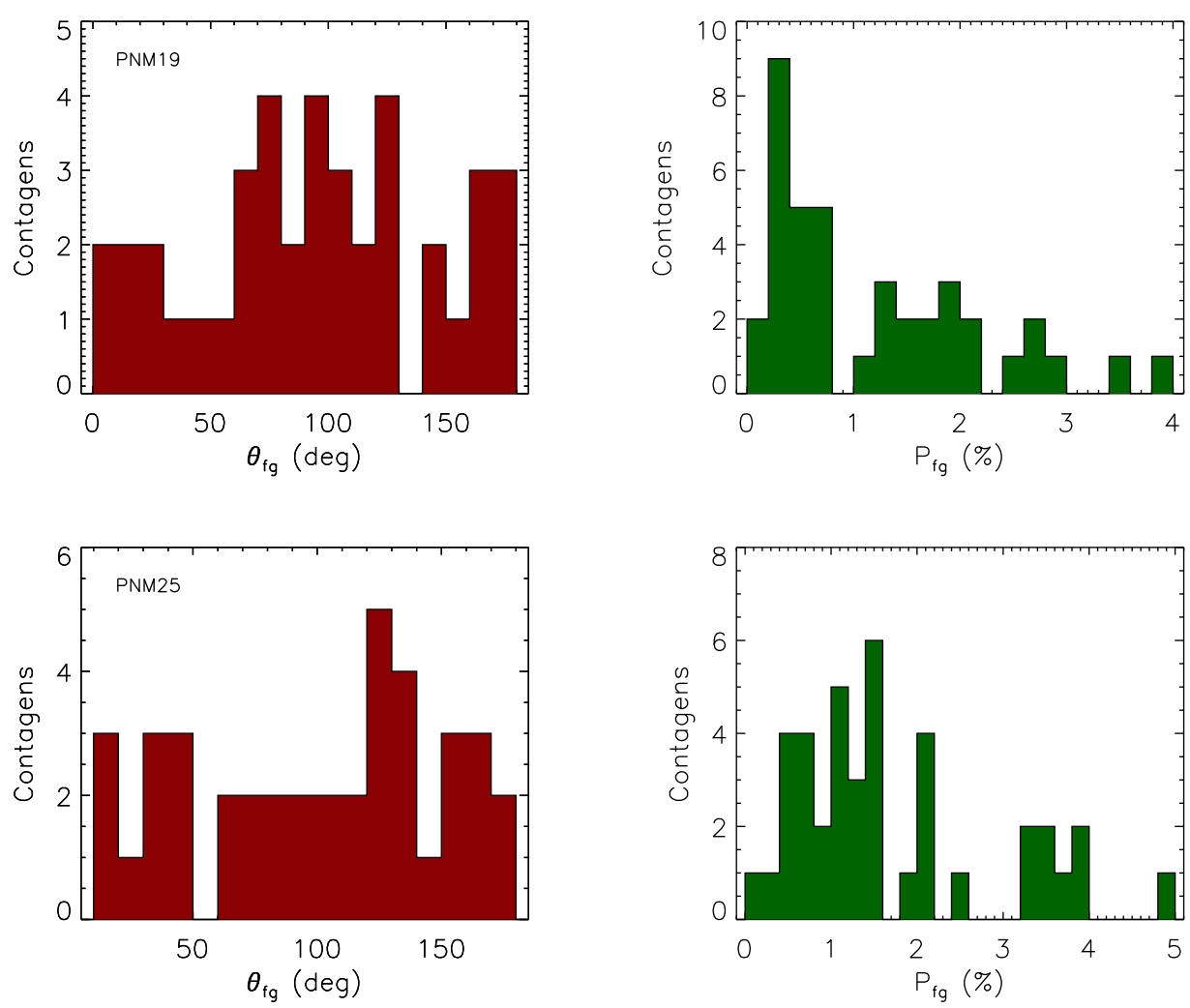

Figura A.5: Campos com nenhum padrão para $\theta$. Histogramas para o ângulo de polarização e a sua intensidade. 


\section{A.4 Campos Filtrados por $m_{V}$}
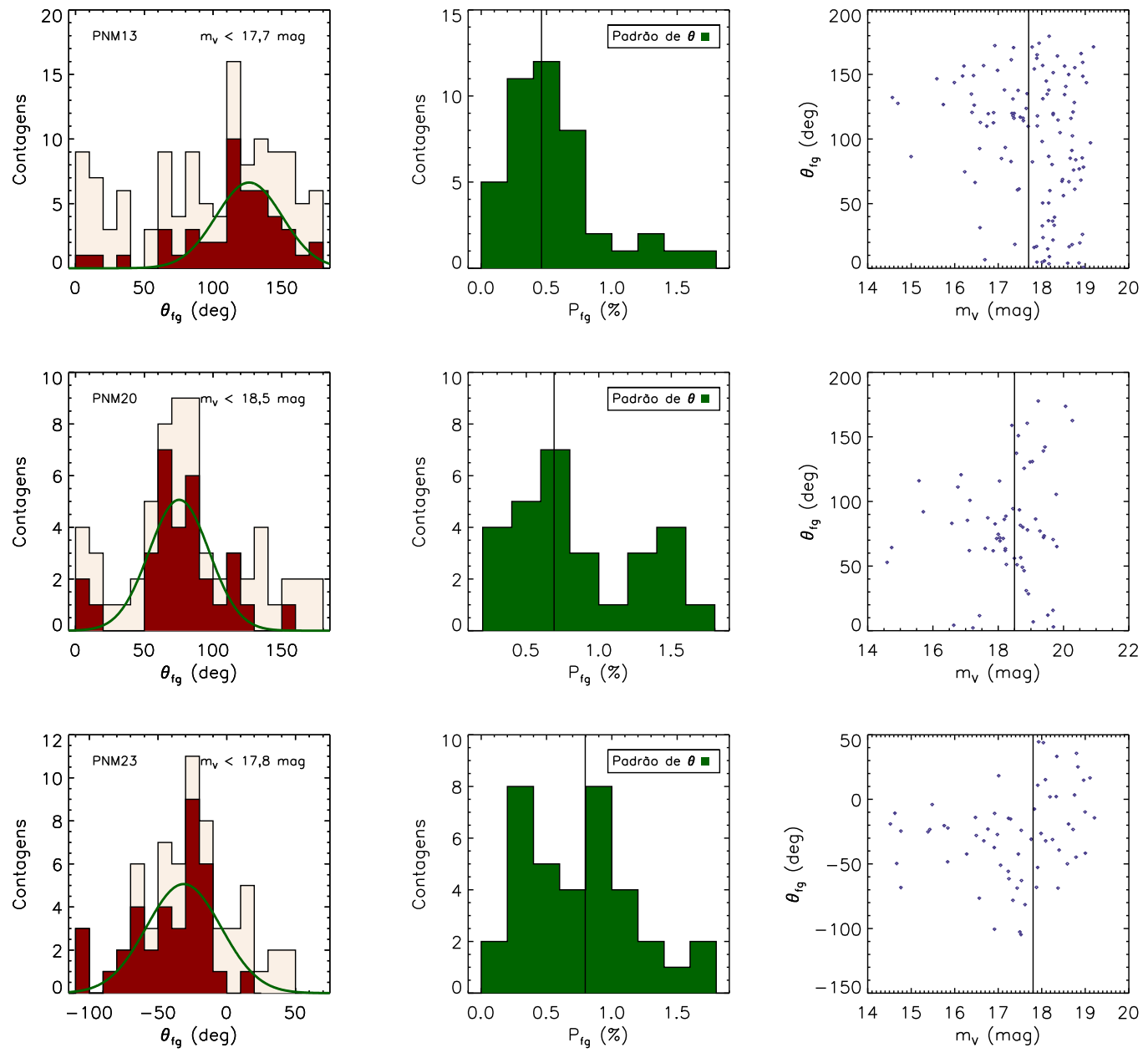

Figura A.6: Campos filtrados por $m_{V}$ para a visualização do padrão de $\theta$. Histogramas para o ângulo de polarização e a sua intensidade, e gráfico de $\theta$ vs $m_{V}$. A linha vertical no histograma de $P$ marca a sua mediana, e no gráfico de $\theta$ vs $m_{V}$ destaca a magnitude de corte. O histograma em rosa apresenta as estrelas para toda a faixa de magnitudes. 


\section{A.5 Campos com Estrelas Excluídas}
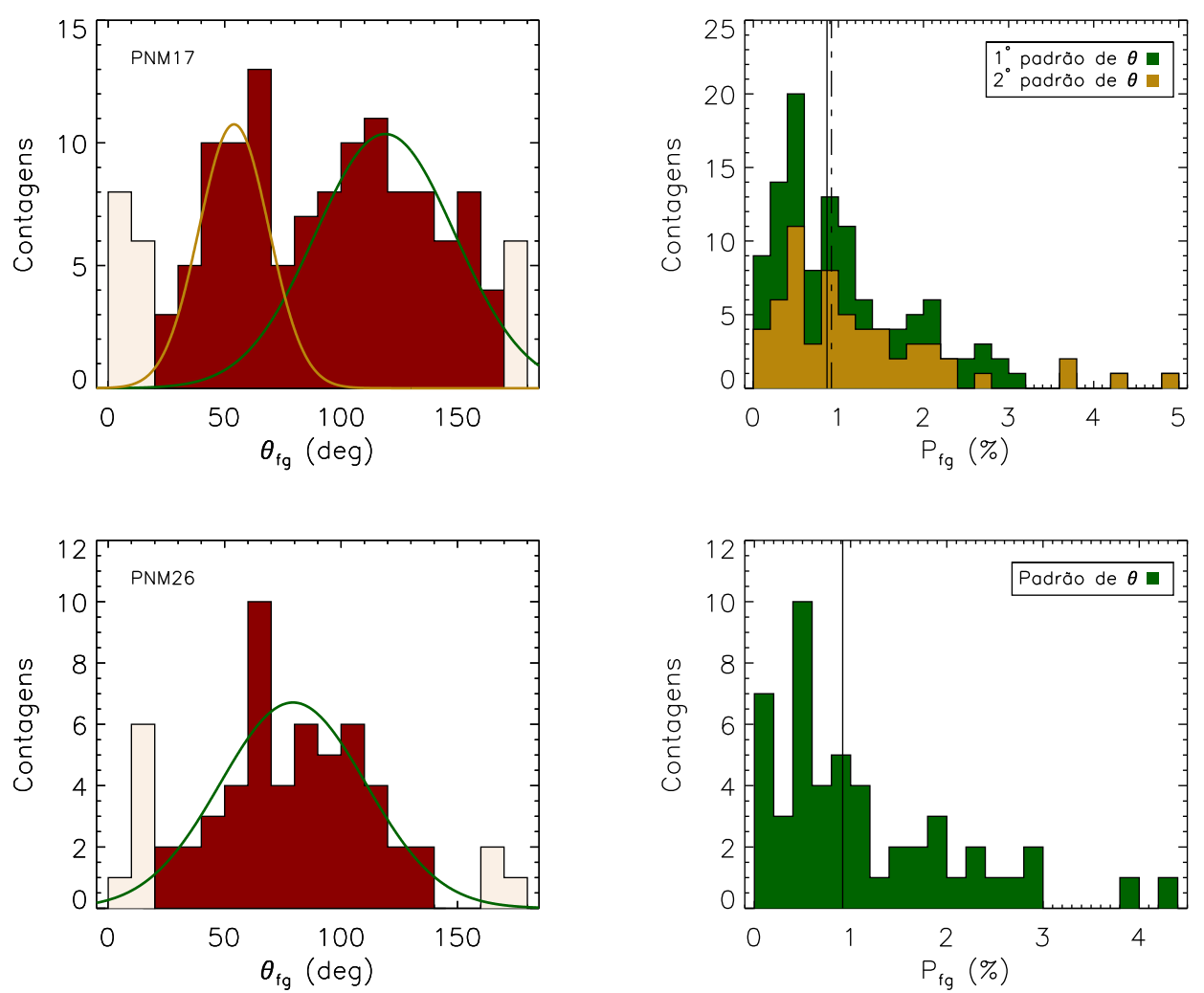

Figura A.7: Campos com estrelas excluídas para o ajuste do padrão de $\theta$. Histogramas para o ângulo de polarização e a sua intensidade. As linhas verticais no histograma de $P$ marcam as medianas para o primeiro (linha sólida) e segundo padrão (linha sólida-pontilhada). Os bins em rosa claro, no histograma de $\theta$, correspondem a estrelas que não foram consideradas no ajuste. 


\section{A.6 Tabela com Parâmetros dos Padrões}

Tabela A.1 - Parâmetros dos padrões de polarização. $\left(\theta_{1}, \delta \theta_{1}\right.$ e $\left.P_{1}\right)$ corresponde ao primeiro padrão, e $\left(\theta_{2}, \delta \theta_{2}\right.$ e $\left.P_{2}\right)$ ao segundo.

\begin{tabular}{|c|c|c|c|c|c|c|}
\hline PNM & $\theta_{1}$ (graus) & $\delta \theta_{1}$ (graus) & $P_{1}(\%)$ & $\theta_{2}$ (graus) & $\delta \theta_{2}$ (graus) & $P_{2}(\%)$ \\
\hline 01 & $(120,90 \pm 0,28)$ & $(21,28 \pm 0,29)$ & 0,90 & $(46,3 \pm 1,1)$ & $(22,9 \pm 1,2)$ & 0,85 \\
\hline 02 & $(58,39 \pm 0,73)$ & $(40,93 \pm 0,90)$ & 0,62 & $(150,0 \pm 1,6)$ & $(19,5 \pm 2,2)$ & 0,66 \\
\hline 03 & $(102,16 \pm 0,55)$ & $(46,08 \pm 0,61)$ & 1,0 & - & - & - \\
\hline 04 & $(169,2 \pm 1,6)$ & $(39,7 \pm 1,9)$ & 0,87 & $(65,5 \pm 2,0)$ & $(10,8 \pm 2,2)$ & 1,0 \\
\hline 05 & - & - & - & - & - & - \\
\hline 06 & $(100,6 \pm 2,1)$ & $(71,9 \pm 3,2)$ & 1,2 & - & - & - \\
\hline 07 & $(84,7 \pm 1,6)$ & $(43,0 \pm 2,2)$ & 1,0 & $(178,85 \pm 0,73)$ & $(25,28 \pm 0,72)$ & 1,0 \\
\hline 08 & $(73,69 \pm 0,38)$ & $(37,03 \pm 0,51)$ & 0,87 & $(154,79 \pm 0,66)$ & $(21,76 \pm 0,88)$ & 0,93 \\
\hline 09 & $(67,51 \pm 0,16)$ & $(18,84 \pm 0,16)$ & 0,98 & - & - & - \\
\hline 10 & $(142,54 \pm 0,31)$ & $(26,45 \pm 0,36)$ & 0,94 & $(54,97 \pm 0,60)$ & $(22,67 \pm 0,72)$ & 1,0 \\
\hline 11 & $(158,29 \pm 0,17)$ & $(17,17 \pm 0,17)$ & 1,4 & - & - & - \\
\hline 12 & $(149,6 \pm 1,4)$ & $(18,2 \pm 1,4)$ & 0,64 & - & - & - \\
\hline 13 & $(126,2 \pm 2,5)$ & $(24,1 \pm 2,6)$ & 0,46 & - & - & - \\
\hline 14 & - & - & - & - & - & - \\
\hline 15 & $(144,5 \pm 1,0)$ & $(27,2 \pm 1,1)$ & 1,1 & - & - & - \\
\hline 16 & $(134,5 \pm 4,8)$ & $(25,6 \pm 4,6)$ & 0,89 & $(66,8 \pm 6,4)$ & $(22,8 \pm 7,0)$ & 0,72 \\
\hline 17 & $(118,9 \pm 2,6)$ & $(30,0 \pm 3,2)$ & 0,87 & $(54,0 \pm 1,6)$ & $(15,0 \pm 1,6)$ & 0,92 \\
\hline 18 & $(88,5 \pm 2,9)$ & $(59,5 \pm 3,7)$ & 1,1 & - & - & - \\
\hline 19 & - & - & - & - & - & - \\
\hline 20 & $(75,3 \pm 3,1)$ & $(21,8 \pm 3,1)$ & 0,69 & - & - & - \\
\hline 21 & $(159,57 \pm 0,49)$ & $(7,51 \pm 0,42)$ & 1,7 & - & - & - \\
\hline 22 & $(157,8 \pm 4,3)$ & $(35,2 \pm 5,4)$ & 0,96 & $(75 \pm 14)$ & $(21 \pm 15)$ & 0,89 \\
\hline 23 & $(148,3 \pm 2,9)$ & $(23,7 \pm 3,0)$ & 0,74 & - & - & - \\
\hline 24 & $(150,1 \pm 2,4)$ & $(27,9 \pm 2,4)$ & 1,3 & - & - & - \\
\hline 25 & - & - & - & - & - & - \\
\hline 26 & $(79,4 \pm 2,9)$ & $(31,3 \pm 3,2)$ & 0,92 & - & - & - \\
\hline 27 & $(69,4 \pm 2,6)$ & $(22,7 \pm 2,9)$ & 0,69 & $(116,2 \pm 1,0)$ & $(7,8 \pm 1,2)$ & 0,67 \\
\hline 28 & $(69,87 \pm 0,77)$ & $(13,90 \pm 0,77)$ & 0,82 & - & - & - \\
\hline
\end{tabular}


Apêndice B

\section{Mapas de Polarização}

Neste apêndice, estão apresentados os mapas de polarização para cada campo. Foi utilizado o catálogo com polarização corrigida pelo foreground obtido neste trabalho, e estrelas com $P / \sigma_{P}>3$. Os vetores foram sobrepostos a imagens do Spitzer/MIPS em $160 \mu \mathrm{m}$. Podem ser visualizadas nos mapas as localizações de objetos relacionados ao MI, presentes em cada campo. Também é apresentada uma tabela com a lista dos objetos.

\section{B.1 Mapas de Polarização por Campo}

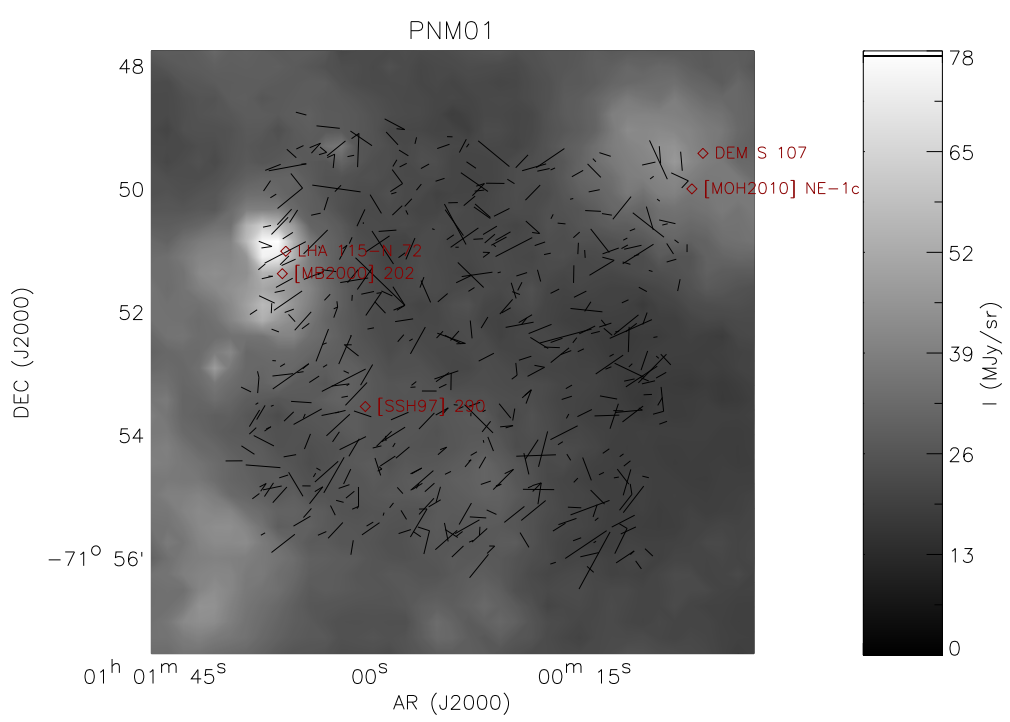

Figura B.1: Mapa de polarização sobreposto a uma imagem do Spitzer/MIPS em $160 \mu \mathrm{m}$. Os diamantes vermelhos representam a localização da coordenada central de estruturas do MI, seguidos por seus nomes. 

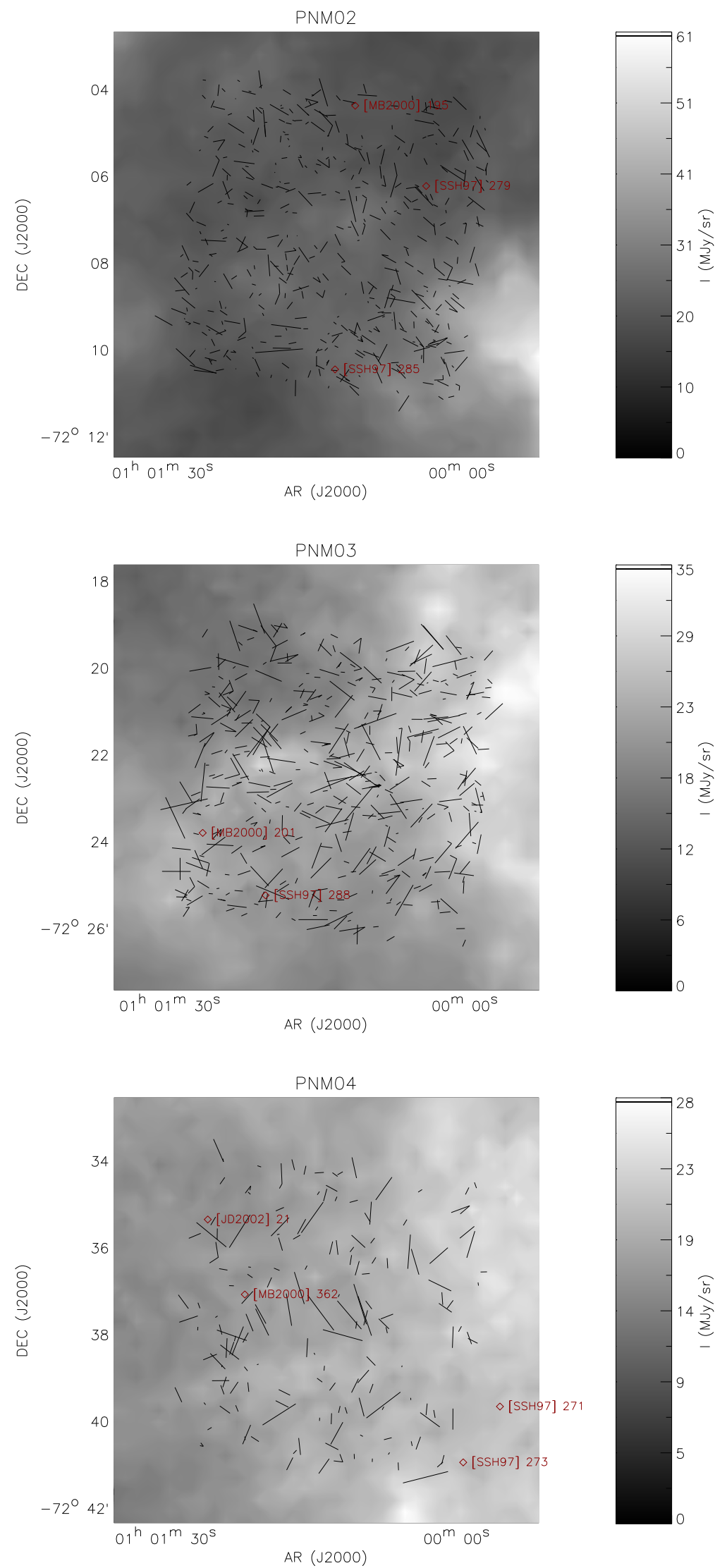

Figura B.2: Mapas de polarização sobrepostos a uma imagem do Spitzer/MIPS em $160 \mu \mathrm{m}$. Os diamantes vermelhos representam a localização da coordenada central de estruturas do MI, seguidos por seus nomes (continuação). 

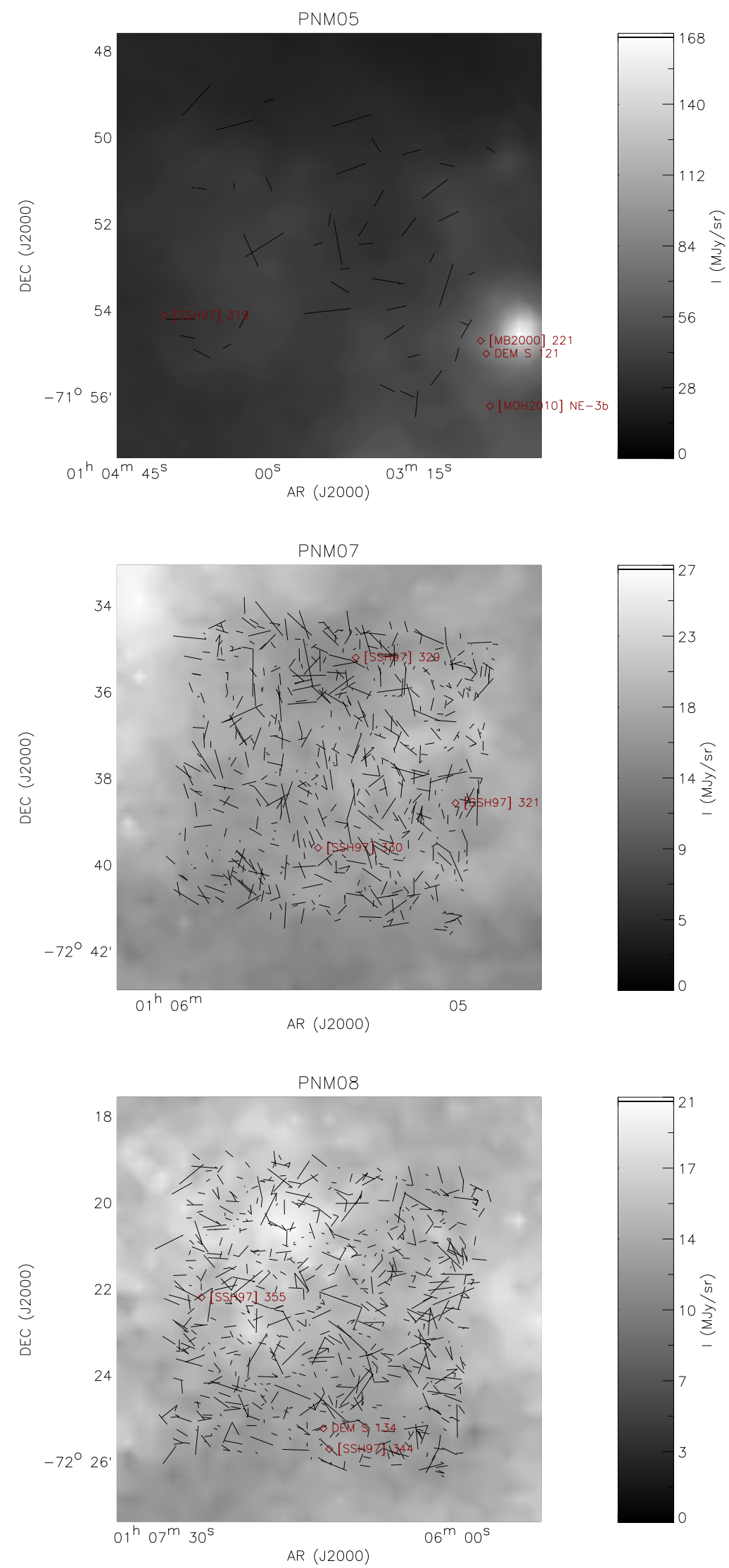

Figura B.3: Mapas de polarização sobrepostos a uma imagem do Spitzer/MIPS em $160 \mu \mathrm{m}$. Os diamantes vermelhos representam a localização da coordenada central de estruturas do MI, seguidos por seus nomes (continuação). 

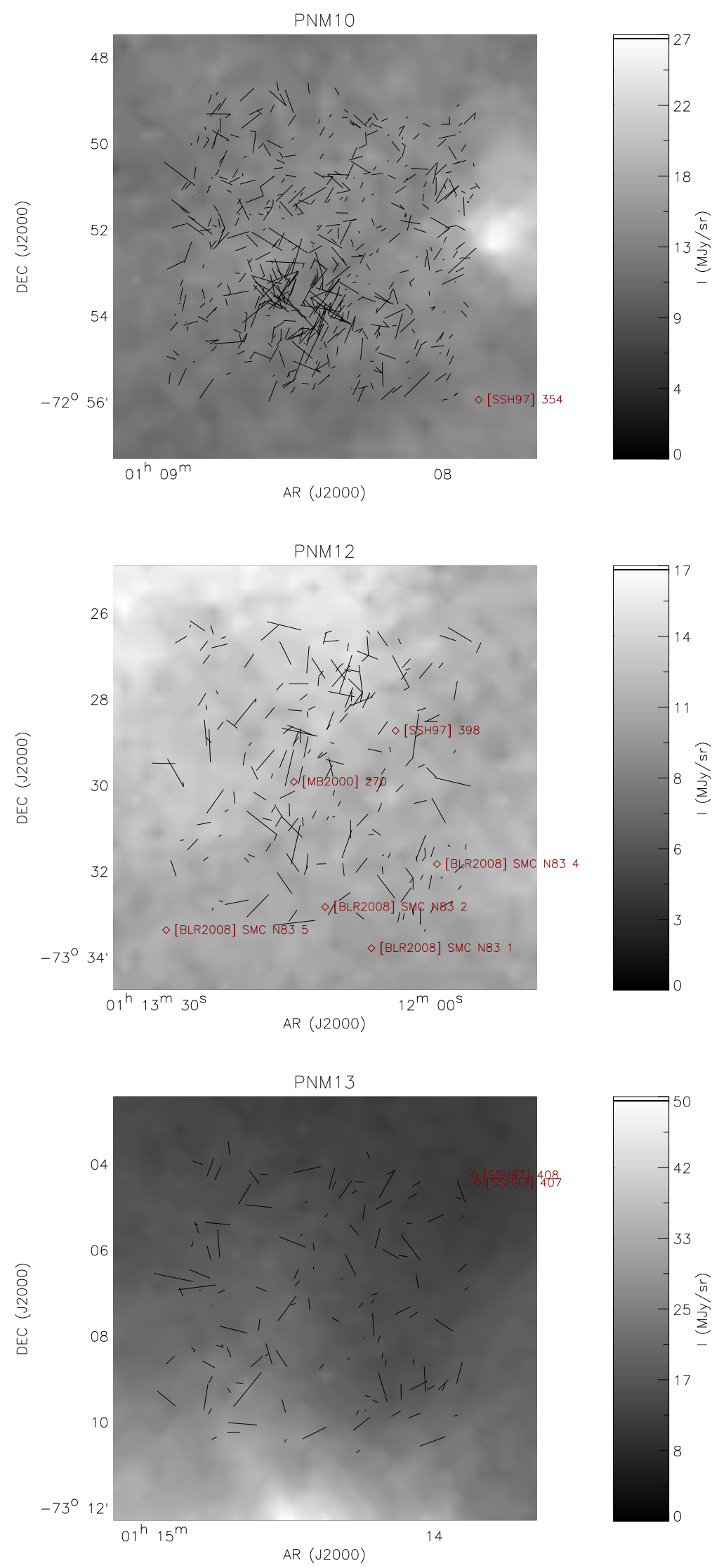

Figura B.4: Mapas de polarização sobrepostos a uma imagem do Spitzer/MIPS em $160 \mu \mathrm{m}$. Os diamantes vermelhos representam a localização da coordenada central de estruturas do MI, seguidos por seus nomes (continuação). 

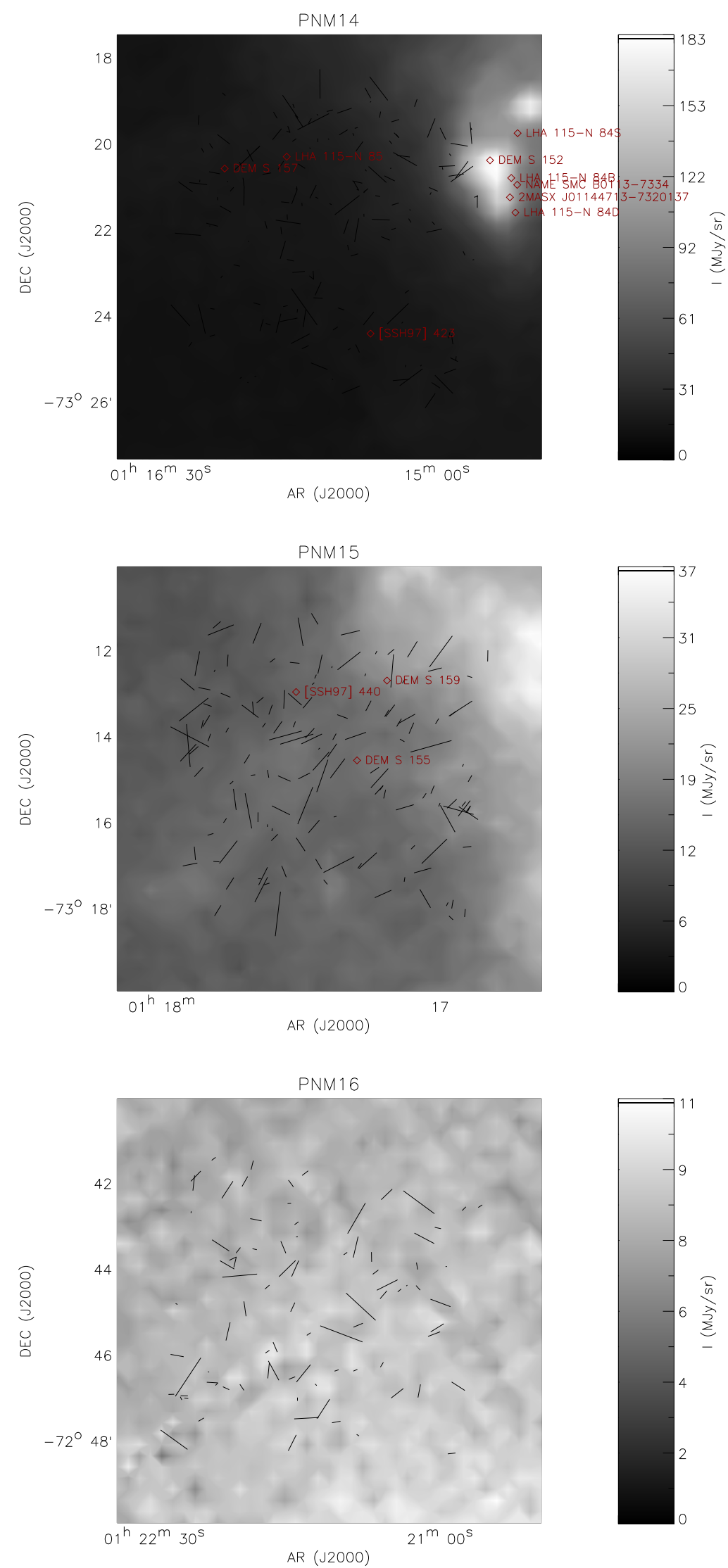

Figura B.5: Mapas de polarização sobrepostos a uma imagem do Spitzer/MIPS em $160 \mu \mathrm{m}$. Os diamantes vermelhos representam a localização da coordenada central de estruturas do MI, seguidos por seus nomes (continuação). 

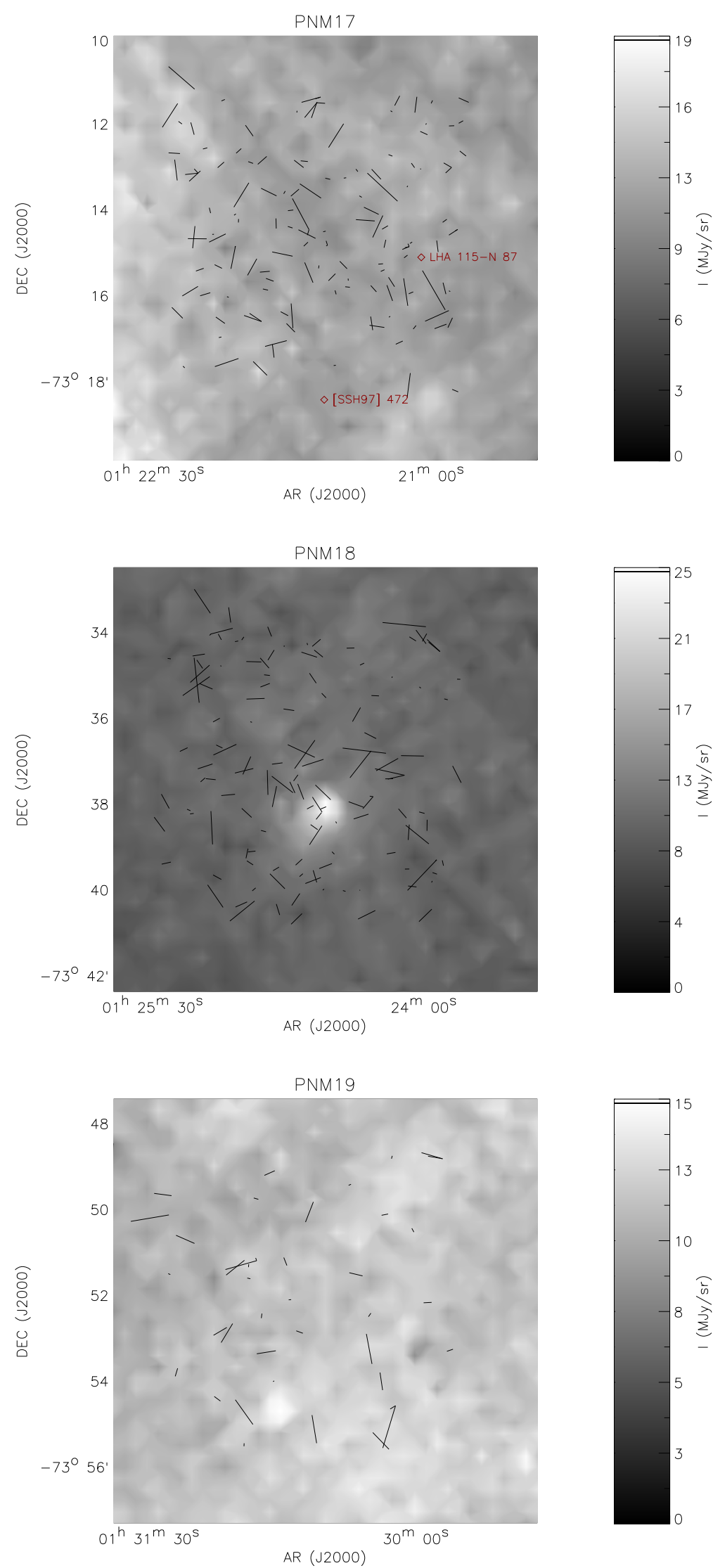

Figura B.6: Mapas de polarização sobrepostos a uma imagem do Spitzer/MIPS em $160 \mu \mathrm{m}$. Os diamantes vermelhos representam a localização da coordenada central de estruturas do MI, seguidos por seus nomes (continuação). 

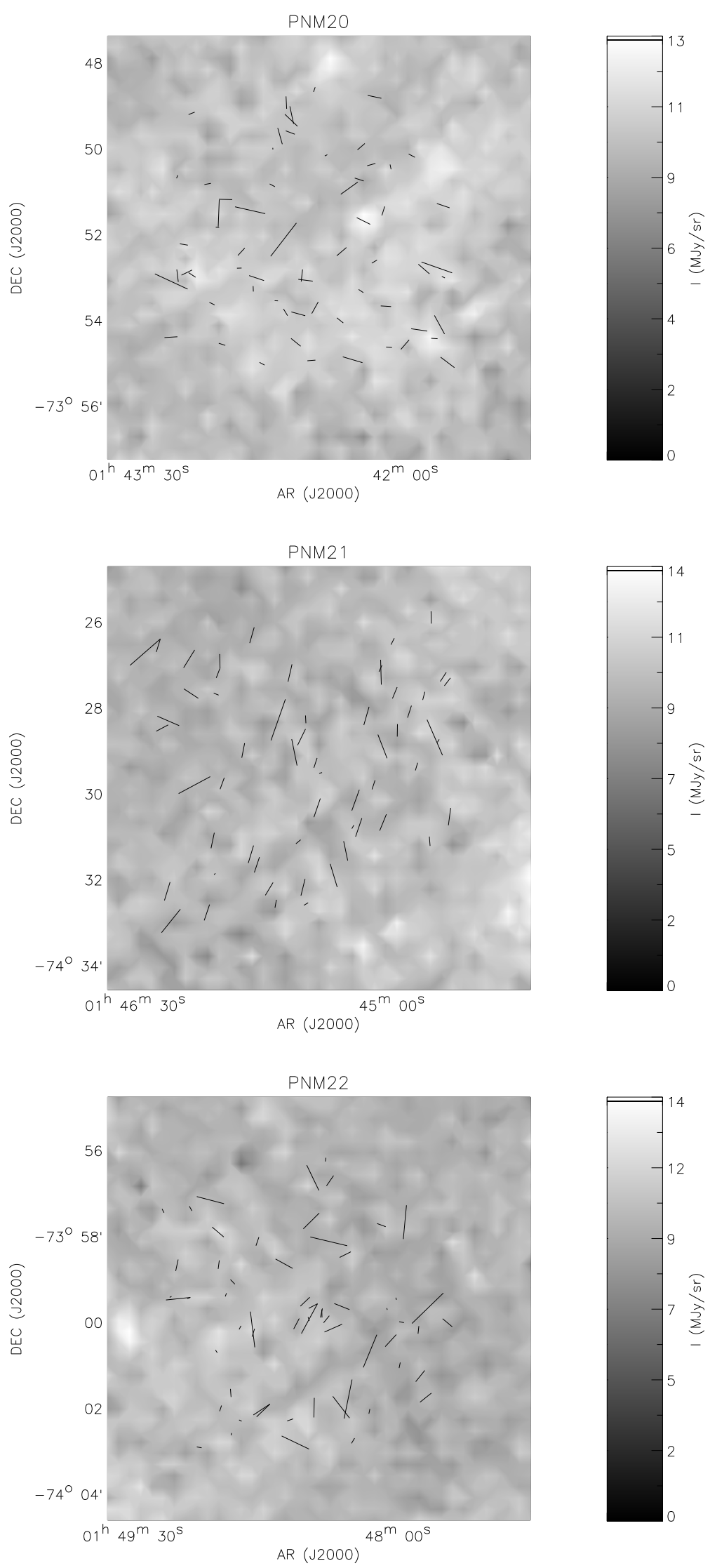

Figura B.7: Mapas de polarização sobrepostos a uma imagem do Spitzer/MIPS em $160 \mu \mathrm{m}$. Os diamantes vermelhos representam a localização da coordenada central de estruturas do MI, seguidos por seus nomes (continuação). 

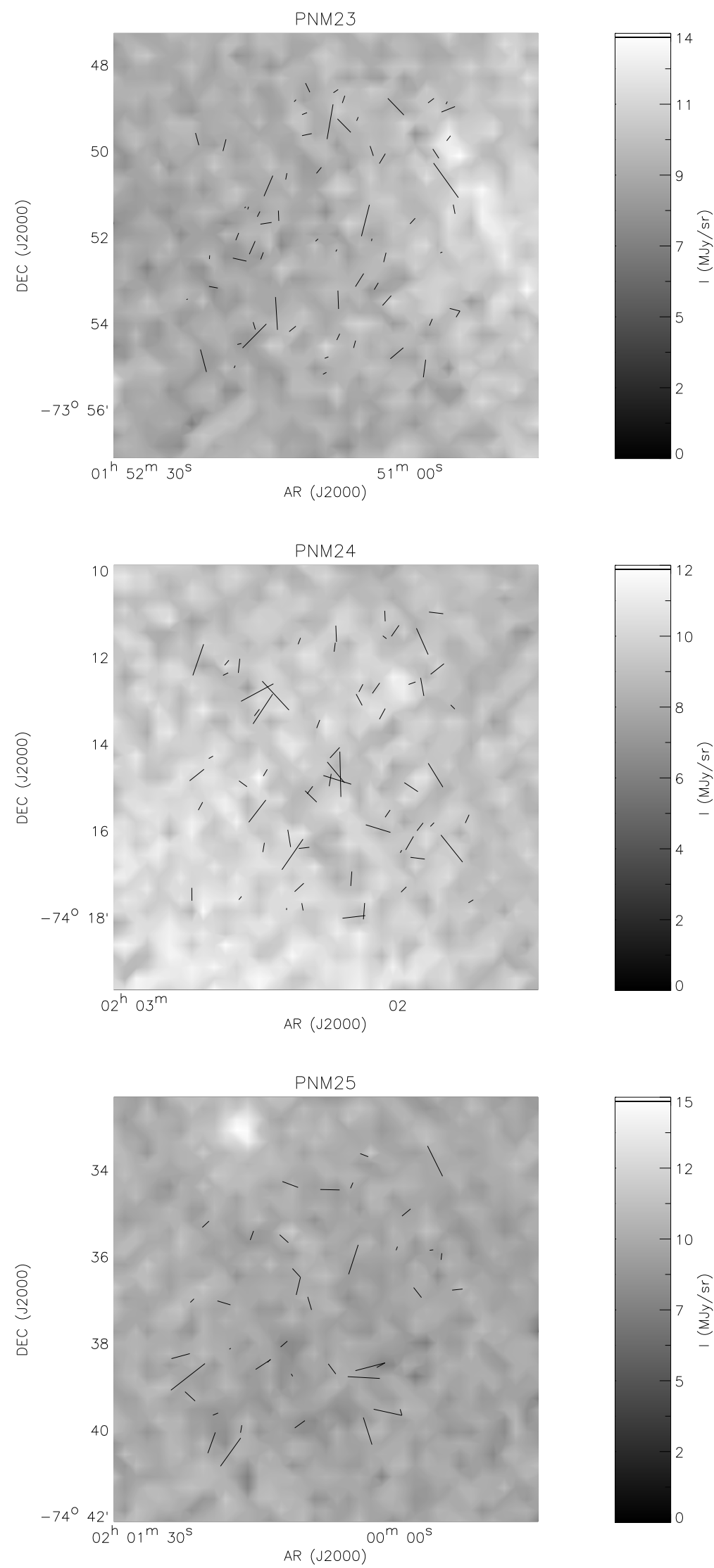

Figura B.8: Mapas de polarização sobrepostos a uma imagem do Spitzer/MIPS em $160 \mu \mathrm{m}$. Os diamantes vermelhos representam a localização da coordenada central de estruturas do MI, seguidos por seus nomes (continuação). 

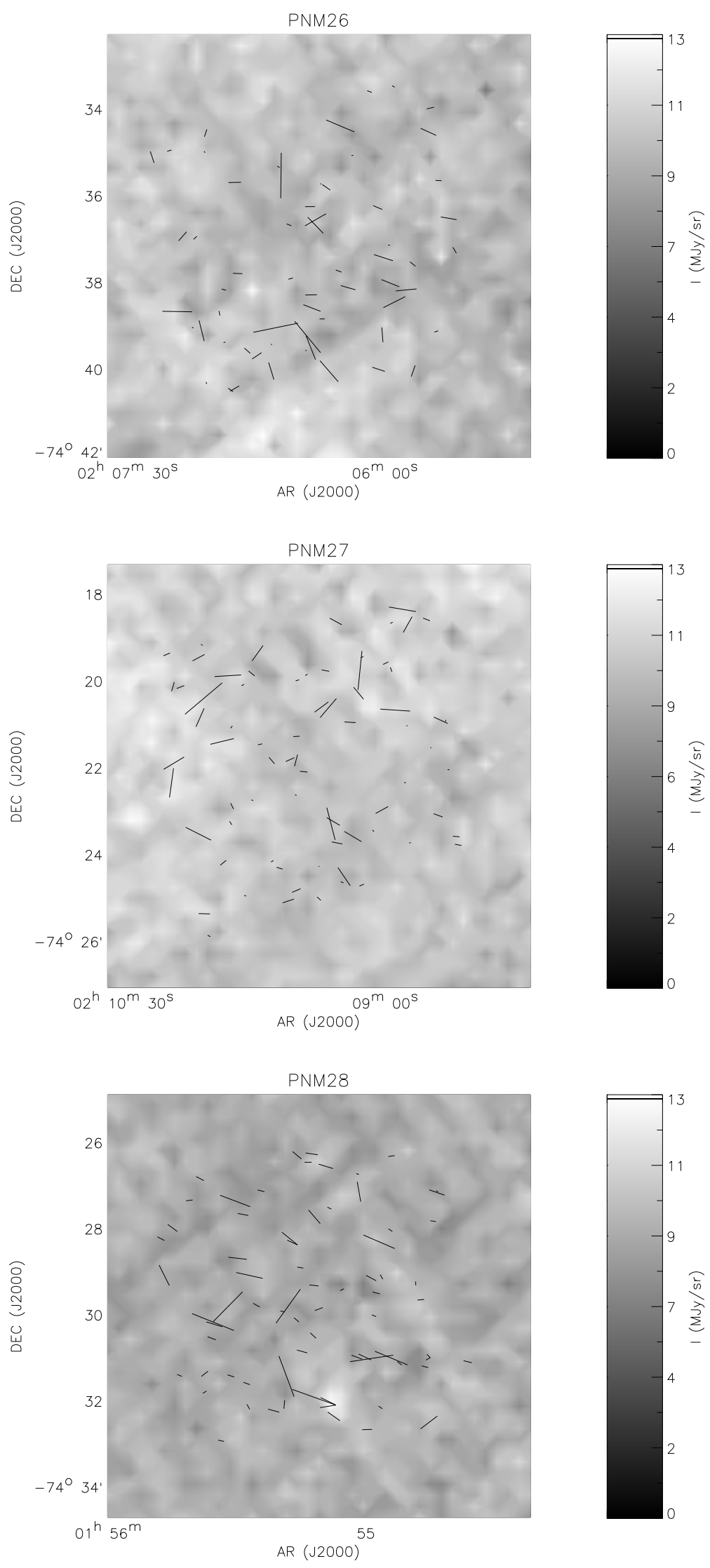

Figura B.9: Mapas de polarização sobrepostos a uma imagem do Spitzer/MIPS em $160 \mu \mathrm{m}$. Os diamantes vermelhos representam a localização da coordenada central de estruturas do MI, seguidos por seus nomes (continuação). 


\section{B.2 Tabela com Objetos}

Tabela B.1 - Lista de objetos relacionados ao MI, presentes em cada campo CCD.

\begin{tabular}{|c|c|c|c|c|}
\hline PNM & Nome & AR (J2000) & DEC (J2000) & Tipo \\
\hline \multirow[t]{5}{*}{01} & DEM S 107 & 01:00:08.7 & $-71: 48: 04$ & Região HII \\
\hline & [MOH2010] NE-1c & 01:00:10.0 & $-71: 48: 40$ & Nuvem Molecular \\
\hline & [SSH97] 290 & 01:01:12 & $-71: 52.9$ & Shell \\
\hline & LHA $115-\mathrm{N} 72$ & 01:01:32.5 & $-71: 50: 43$ & Região HII \\
\hline & [MB2000] 202 & 01:01:32.9 & $-71: 50: 55$ & Objeto com linha de emissão \\
\hline \multirow[t]{3}{*}{02} & [SSH97] 279 & 01:00:26 & $-72: 05.1$ & Shell \\
\hline & [MB2000] 195 & 01:00:50.4 & $-72: 03: 28$ & Objeto com linha de emissão \\
\hline & [SSH97] 285 & 01:00:46 & $-72: 09.6$ & Shell \\
\hline \multirow[t]{2}{*}{03} & [MB2000] 201 & 01:01:30.6 & $-72: 23: 21$ & Objeto com linha de emissão \\
\hline & [SSH97] 288 & 01:01:09 & $-72: 24.6$ & Shell \\
\hline \multirow[t]{4}{*}{04} & [SSH97] 271 & $00: 59: 56$ & $-72: 38.3$ & Shell \\
\hline & [JD2002] 21 & 01:01:33.6 & $-72: 34: 53$ & Nebulosa Planetária \\
\hline & [SSH97] 273 & 01:00:05 & $-72: 39.7$ & Shell \\
\hline & [MB2000] 362 & 01:01:19.2 & $-72: 36: 30$ & Objeto com linha de emissão \\
\hline \multirow[t]{4}{*}{05} & [MB2000] 221 & 01:03:05.1 & $-71: 53: 31$ & Objeto com linha de emissão \\
\hline & [MOH2010] NE-3b & 01:03:00.0 & $-71: 55: 00$ & Nuvem Molecular \\
\hline & DEM S 121 & 01:03:03 & $-71: 53.8$ & Região HII \\
\hline & [SSH97] 319 & 01:04:40 & $-71: 53.8$ & Shell \\
\hline \multirow[t]{9}{*}{06} & [MB2000] 222 & 01:03:07.4 & $-72: 06: 25$ & Objeto com linha de emissão \\
\hline & {$[\mathrm{H} 74] 44$} & 01:03.0 & $-72: 10$ & Nuvem Escura (Nebulosa) \\
\hline & [MOH2010] NE-3g & 01:03:10.0 & $-72: 03: 50$ & Nuvem Molecular \\
\hline & DEM S 119 & 01:03:01.9 & $-72: 05: 17$ & Região HII \\
\hline & LHA $115-\mathrm{N} 76 \mathrm{~B}$ & 01:03:08.0 & $-72: 06: 24$ & Região HII \\
\hline & LHA $115-\mathrm{N} 76 \mathrm{~A}$ & 01:03:48.9 & $-72: 03: 52$ & Região HII \\
\hline & [SSH97] 309 & 01:03:03 & $-72: 03.2$ & Shell \\
\hline & SNR B0101-72.6 & 01:03:17 & $-72: 09.7$ & Remanescente de Supernova \\
\hline & LHA $115-\mathrm{N} 76 \mathrm{C}$ & 01:03:54.1 & $-72: 08: 23$ & Objeto com linha de emissão \\
\hline \multirow[t]{3}{*}{07} & [SSH97] 329 & 01:05:18 & $-72: 34.4$ & Shell \\
\hline & [SSH97] 330 & 01:05:23 & $-72: 38.9$ & Shell \\
\hline & [SSH97] 321 & 01:04:42 & $-72: 37.5$ & Shell \\
\hline \multirow[t]{3}{*}{08} & DEM S 134 & $01: 06: 48.5$ & $-72: 24: 32$ & Região HII \\
\hline & [SSH97] 344 & 01:06:46 & $-72: 25.0$ & Shell \\
\hline & [SSH97] 355 & 01:07:30 & $-72: 21.8$ & Shell \\
\hline \multirow[t]{3}{*}{09} & [SSH97] 367 & 01:08:43 & $-72: 38.1$ & Shell \\
\hline & [SSH97] 358 & 01:07:33 & $-72: 39.9$ & Shell \\
\hline & LHA $115-\mathrm{N} 79$ & $01: 07: 36.7$ & $-72: 34: 47$ & Objeto com linha de emissão \\
\hline 10 & [SSH97] 354 & $01: 07: 25$ & $-72: 54.9$ & Shell \\
\hline \multirow[t]{3}{*}{11} & [SSH97] 376 & 01:09:29 & $-72: 57.4$ & Shell \\
\hline & [SSH97] 386 & 01:10:31 & $-73: 03.1$ & Shell \\
\hline & [SSH97] 373 & 01:09:20 & $-73: 01.9$ & Shell \\
\hline
\end{tabular}


Tabela B.1 - Continuação

\begin{tabular}{|c|c|c|c|c|}
\hline PNM & Nome & AR (J2000) & DEC (J2000) & Tipo \\
\hline & [SSH97] 387 & $01: 10: 33$ & $-73: 01.6$ & Shell \\
\hline \multirow[t]{6}{*}{12} & [BLR2008] SMC N83 4 & 01:12:05.8 & $-73: 31: 01$ & Nuvem Molecular \\
\hline & [MB2000] 270 & $01: 12: 54.9$ & $-73: 29: 23$ & Objeto com linha de emissão \\
\hline & [SSH97] 398 & $01: 12: 23$ & $-73: 28.0$ & Shell \\
\hline & [BLR2008] SMC N83 1 & $01: 12: 25.0$ & $-73: 33: 06$ & Nuvem Molecular \\
\hline & [BLR2008] SMC N83 5 & $01: 13: 32.9$ & $-73: 33: 04$ & Nuvem Molecular \\
\hline & [BLR2008] SMC N83 2 & 01:12:41.3 & $-73: 32: 14$ & Nuvem Molecular \\
\hline \multirow[t]{2}{*}{13} & [SSH97] 408 & 01:13:30 & $-73: 03.6$ & Shell \\
\hline & [SSH97] 407 & 01:13:29 & $-73: 03.6$ & Shell \\
\hline \multirow[t]{9}{*}{14} & 2MASX J01144713-7320137 & 01:14:47.132 & $-73: 20: 13.80$ & Nebulosa Planetária \\
\hline & LHA $115-\mathrm{N} 84 \mathrm{~B}$ & 01:14:47.17 & $-73: 19: 46.7$ & Objeto com linha de emissão \\
\hline & LHA $115-\mathrm{N} 84 \mathrm{D}$ & $01: 14: 44.827$ & $-73: 20: 44.80$ & Objeto com linha de emissão \\
\hline & DEM S 152 & $01: 14: 54.1$ & $-73: 19: 45$ & Região HII \\
\hline & LHA $115-\mathrm{N} 85$ & 01:16.0 & $-73: 20$ & Objeto com linha de emissão \\
\hline & NAME SMC B0113-7334 & $01: 14: 44.9$ & $-73: 20: 06$ & Região HII \\
\hline & LHA $115-\mathrm{N} 84 \mathrm{~S}$ & $01: 14: 46$ & $-73: 18.9$ & Objeto com linha de emissão \\
\hline & DEM S 157 & $01: 16: 20$ & $-73: 20.2$ & Região HII \\
\hline & [SSH97] 423 & $01: 15: 29$ & $-73: 23.8$ & Shell \\
\hline \multirow[t]{3}{*}{15} & [SSH97] 440 & $01: 17: 27$ & $-73: 12.5$ & Shell \\
\hline & DEM S 159 & $01: 16: 58$ & $-73: 12.1$ & Região HII \\
\hline & DEM S 155 & 01:17.1 & $-73: 14$ & Região HII \\
\hline 16 & - & - & - & - \\
\hline \multirow[t]{2}{*}{17} & LHA $115-\mathrm{N} 87$ & 01:21:10.69 & $-73: 14: 34.8$ & Nebulosa Planetária \\
\hline & [SSH97] 472 & $01: 21: 40$ & $-73: 18.0$ & Shell \\
\hline 18 & - & - & - & - \\
\hline 19 & - & - & - & - \\
\hline 20 & - & - & - & - \\
\hline 21 & - & - & - & - \\
\hline 22 & - & - & - & - \\
\hline 23 & - & - & - & - \\
\hline 24 & - & - & - & - \\
\hline 25 & - & - & - & - \\
\hline 26 & - & - & - & - \\
\hline 27 & - & - & - & - \\
\hline 28 & - & - & - & - \\
\hline
\end{tabular}

